\title{
Panel Survey of Young People in Egypt (SYPE) 2014: Generating evidence for policy, programs, and research-Appendix
}

Rania Roushdy

Population Council

Maia Sieverding

Follow this and additional works at: https://knowledgecommons.popcouncil.org/departments_sbsr-pgy

Part of the Demography, Population, and Ecology Commons, Family, Life Course, and Society Commons, and the International Public Health Commons How does access to this work benefit you? Let us know!

\section{Recommended Citation}

Roushdy, Rania and Maia Sieverding. 2015. "Panel Survey of Young People in Egypt (SYPE) 2014:

Generating evidence for policy, programs, and research-Appendix." Cairo: Population Council. 
Table S1 Indicator: Labor force participation rate (broad definition), by gender

\begin{tabular}{|c|c|c|c|c|c|c|c|c|c|c|c|c|c|c|}
\hline \multirow[b]{3}{*}{ Characteristic } & \multirow[b]{3}{*}{ Estimate } & \multirow[b]{3}{*}{ SE } & \multicolumn{3}{|c|}{ Male } & \multirow{3}{*}{$\begin{array}{c}\text { Design } \\
\text { effect }\end{array}$} & \multirow[b]{3}{*}{ Count } & \multirow[b]{3}{*}{ Estimate } & \multirow[b]{3}{*}{ SE } & \multirow{2}{*}{\multicolumn{2}{|c|}{ Female }} & \multirow{3}{*}{$\begin{array}{l}\text { CV } \\
(\%)\end{array}$} & \multirow{3}{*}{$\begin{array}{c}\text { Design } \\
\text { effect }\end{array}$} & \multirow{3}{*}{ Count } \\
\hline & & & \multicolumn{2}{|c|}{ 95\% C.I } & \multirow{2}{*}{$\begin{array}{l}\mathrm{CV} \\
(\%)\end{array}$} & & & & & & & & & \\
\hline & & & Lower & Upper & & & & & & Lower & Upper & & & \\
\hline \multicolumn{15}{|l|}{ Urban-rural residence } \\
\hline Urban & 0.639 & 0.016 & 0.608 & 0.670 & 0.024 & 1.778 & 1,621 & 0.204 & 0.015 & 0.175 & 0.233 & 0.072 & 2.065 & 1,689 \\
\hline Rural & 0.663 & 0.011 & 0.641 & 0.685 & 0.017 & 1.868 & 2,947 & 0.170 & 0.010 & 0.149 & 0.190 & 0.061 & 2.452 & 3,530 \\
\hline Informal urban areas & 0.615 & 0.030 & 0.556 & 0.673 & 0.048 & 1.973 & 492 & 0.159 & 0.024 & 0.113 & 0.206 & 0.148 & 2.231 & 605 \\
\hline \multicolumn{15}{|l|}{ Region } \\
\hline Urban Governorates & 0.661 & 0.018 & 0.625 & 0.697 & 0.028 & 1.600 & 1,025 & 0.231 & 0.017 & 0.200 & 0.265 & 0.073 & 1.561 & 1,038 \\
\hline Urban Lower Egypt & 0.591 & 0.027 & 0.538 & 0.645 & 0.046 & 1.965 & 575 & 0.187 & 0.023 & 0.140 & 0.232 & 0.122 & 2.148 & 640 \\
\hline Rural Lower Egypt & 0.661 & 0.015 & 0.631 & 0.691 & 0.023 & 1.844 & 1,609 & 0.183 & 0.013 & 0.160 & 0.209 & 0.073 & 1.928 & 1,798 \\
\hline Urban Upper Egypt & 0.626 & 0.032 & 0.564 & 0.688 & 0.051 & 1.925 & 287 & 0.118 & 0.025 & 0.070 & 0.166 & 0.211 & 2.517 & 350 \\
\hline Rural Upper Egypt & 0.663 & 0.017 & 0.630 & 0.696 & 0.025 & 1.931 & 1,108 & 0.159 & 0.016 & 0.130 & 0.190 & 0.101 & 3.065 & 1,503 \\
\hline Frontier Governorates & 0.684 & 0.034 & 0.617 & 0.750 & 0.050 & 0.546 & 456 & 0.113 & 0.024 & 0.070 & 0.160 & 0.214 & 0.560 & 495 \\
\hline \multicolumn{15}{|l|}{ Age group } \\
\hline $13-17$ years & 0.210 & 0.015 & 0.180 & 0.241 & 0.073 & 1.578 & 1,089 & 0.038 & 0.007 & 0.024 & 0.053 & 0.195 & 1.685 & 1,102 \\
\hline $18-24$ years & 0.585 & 0.014 & 0.558 & 0.612 & 0.023 & 1.798 & 2,074 & 0.178 & 0.012 & 0.154 & 0.202 & 0.068 & 1.994 & 2,071 \\
\hline $25-29$ years & 0.934 & 0.009 & 0.917 & 0.951 & 0.009 & 1.606 & 1,103 & 0.242 & 0.015 & 0.212 & 0.273 & 0.064 & 1.614 & 1,454 \\
\hline 30-35 years & 0.968 & 0.007 & 0.954 & 0.982 & 0.007 & 1.455 & 791 & 0.260 & 0.016 & 0.229 & 0.291 & 0.060 & 1.226 & 1,197 \\
\hline \multicolumn{15}{|l|}{ Education level } \\
\hline Illiterate & 0.817 & 0.031 & 0.756 & 0.879 & 0.038 & 1.975 & 281 & 0.104 & 0.016 & 0.073 & 0.135 & 0.151 & 1.744 & 823 \\
\hline Read and write & 0.947 & 0.037 & 0.875 & 1.019 & 0.039 & 1.066 & 38 & 0.116 & 0.044 & 0.029 & 0.204 & 0.383 & 1.303 & 83 \\
\hline Primary & 0.883 & 0.016 & 0.851 & 0.915 & 0.018 & 1.254 & 453 & 0.164 & 0.022 & 0.120 & 0.208 & 0.136 & 1.386 & 435 \\
\hline Preparatory & 0.549 & 0.024 & 0.502 & 0.595 & 0.043 & 1.600 & 645 & 0.086 & 0.012 & 0.063 & 0.109 & 0.135 & 1.398 & 870 \\
\hline General secondary & 0.205 & 0.025 & 0.156 & 0.253 & 0.121 & 1.636 & 416 & 0.030 & 0.008 & 0.013 & 0.046 & 0.283 & 1.182 & 515 \\
\hline Vocational secondary & 0.716 & 0.012 & 0.692 & 0.740 & 0.017 & 1.657 & 2,023 & 0.185 & 0.012 & 0.162 & 0.209 & 0.063 & 1.615 & 1,949 \\
\hline Post-secondary institute & 0.693 & 0.036 & 0.622 & 0.764 & 0.052 & 1.243 & 187 & 0.334 & 0.044 & 0.247 & 0.420 & 0.132 & 1.324 & 165 \\
\hline University and above & 0.596 & 0.017 & 0.562 & 0.630 & 0.029 & 1.422 & 1,014 & 0.349 & 0.019 & 0.312 & 0.385 & 0.053 & 1.523 & 981 \\
\hline \multicolumn{15}{|l|}{ Wealth quintile } \\
\hline Lowest & 0.684 & 0.019 & 0.646 & 0.722 & 0.028 & 1.725 & 827 & 0.154 & 0.016 & 0.123 & 0.185 & 0.103 & 1.920 & 1,017 \\
\hline Second & 0.692 & 0.018 & 0.656 & 0.727 & 0.026 & 1.682 & 950 & 0.164 & 0.014 & 0.136 & 0.192 & 0.087 & 1.602 & 1,141 \\
\hline Middle & 0.682 & 0.017 & 0.648 & 0.715 & 0.025 & 1.354 & 983 & 0.148 & 0.013 & 0.121 & 0.174 & 0.090 & 1.416 & 1,163 \\
\hline Fourth & 0.630 & 0.017 & 0.596 & 0.664 & 0.028 & 1.483 & 1,080 & 0.176 & 0.014 & 0.148 & 0.204 & 0.082 & 1.459 & 1,187 \\
\hline Highest & 0.587 & 0.016 & 0.555 & 0.620 & 0.028 & 1.456 & 1,220 & 0.240 & 0.015 & 0.210 & 0.270 & 0.064 & 1.558 & 1,316 \\
\hline
\end{tabular}


Table S2 Indicator: Labor force participation rate (market LF definition), by gender

\begin{tabular}{|c|c|c|c|c|c|c|c|c|c|c|c|c|c|c|}
\hline \multirow[b]{3}{*}{ Characteristic } & \multirow[b]{3}{*}{ Estimate } & \multirow[b]{3}{*}{ SE } & \multicolumn{3}{|c|}{ Male } & \multirow{3}{*}{$\begin{array}{c}\text { Design } \\
\text { effect }\end{array}$} & \multirow[b]{3}{*}{ Count } & \multirow[b]{3}{*}{ Estimate } & \multirow[b]{3}{*}{ SE } & \multirow{2}{*}{\multicolumn{2}{|c|}{ Female }} & \multirow{3}{*}{$\begin{array}{l}\text { CV } \\
(\%)\end{array}$} & \multirow{3}{*}{$\begin{array}{l}\text { Design } \\
\text { effect }\end{array}$} & \multirow[b]{3}{*}{ Count } \\
\hline & & & \multicolumn{2}{|c|}{ 95\% C.I } & \multirow{2}{*}{$\begin{array}{l}\text { CV } \\
(\%)\end{array}$} & & & & & & & & & \\
\hline & & & Lower & Upper & & & & & & Lower & Upper & & & \\
\hline \multicolumn{15}{|l|}{ Urban-rural residence } \\
\hline Urban & 0.618 & 0.016 & 0.586 & 0.650 & 0.026 & 1.855 & 1,621 & 0.181 & 0.014 & 0.154 & 0.207 & 0.075 & 1.903 & 1,689 \\
\hline Rural & 0.636 & 0.012 & 0.613 & 0.659 & 0.018 & 1.935 & 2,947 & 0.134 & 0.009 & 0.117 & 0.151 & 0.064 & 2.081 & 3,530 \\
\hline Informal urban areas & 0.559 & 0.034 & 0.493 & 0.626 & 0.060 & 2.457 & 492 & 0.112 & 0.020 & 0.072 & 0.151 & 0.179 & 2.165 & 605 \\
\hline \multicolumn{15}{|l|}{ Region } \\
\hline Urban Governorates & 0.638 & 0.019 & 0.600 & 0.675 & 0.030 & 1.705 & 1,025 & 0.206 & 0.017 & 0.17 & 0.238 & 0.081 & 1.637 & 1,038 \\
\hline Urban Lower Egypt & 0.553 & 0.028 & 0.498 & 0.608 & 0.051 & 2.023 & 575 & 0.143 & 0.019 & 0.11 & 0.181 & 0.134 & 1.878 & 640 \\
\hline Rural Lower Egypt & 0.626 & 0.016 & 0.595 & 0.657 & 0.025 & 1.918 & 1,609 & 0.141 & 0.011 & 0.12 & 0.162 & 0.074 & 1.468 & 1,798 \\
\hline Urban Upper Egypt & 0.596 & 0.036 & 0.525 & 0.668 & 0.061 & 2.453 & 287 & 0.098 & 0.021 & 0.06 & 0.139 & 0.212 & 2.08 & 350 \\
\hline Rural Upper Egypt & 0.645 & 0.017 & 0.611 & 0.679 & 0.027 & 1.993 & 1,108 & 0.129 & 0.014 & 0.1 & 0.156 & 0.109 & 2.769 & 1,503 \\
\hline Frontier Governorates & 0.658 & 0.038 & 0.582 & 0.733 & 0.058 & 0.672 & 456 & 0.098 & 0.024 & 0.05 & 0.144 & 0.240 & 0.604 & 495 \\
\hline \multicolumn{15}{|l|}{ Age group } \\
\hline $13-17$ years & 0.194 & 0.015 & 0.165 & 0.223 & 0.076 & 1.542 & 1,089 & 0.028 & 0.007 & 0.015 & 0.040 & 0.240 & 1.825 & 1,102 \\
\hline $18-24$ years & 0.550 & 0.014 & 0.522 & 0.577 & 0.025 & 1.825 & 2,074 & 0.136 & 0.010 & 0.115 & 0.156 & 0.077 & 1.843 & 2,071 \\
\hline $25-29$ years & 0.902 & 0.011 & 0.880 & 0.924 & 0.012 & 1.829 & 1,103 & 0.210 & 0.015 & 0.181 & 0.238 & 0.070 & 1.590 & 1,454 \\
\hline 30-35 years & 0.951 & 0.010 & 0.932 & 0.971 & 0.010 & 1.805 & 791 & 0.219 & 0.015 & 0.190 & 0.247 & 0.067 & 1.203 & 1,197 \\
\hline \multicolumn{15}{|l|}{ Education level } \\
\hline Illiterate & 0.791 & 0.031 & 0.730 & 0.852 & 0.039 & 1.781 & 281 & 0.092 & 0.015 & 0.063 & 0.122 & 0.164 & 1.786 & 823 \\
\hline Read and write & 0.947 & 0.037 & 0.875 & 1.019 & 0.039 & 1.066 & 38 & 0.090 & 0.042 & 0.008 & 0.172 & 0.465 & 1.441 & 83 \\
\hline Primary & 0.857 & 0.018 & 0.821 & 0.893 & 0.021 & 1.321 & 453 & 0.139 & 0.020 & 0.099 & 0.179 & 0.148 & 1.346 & 435 \\
\hline Preparatory & 0.532 & 0.024 & 0.485 & 0.578 & 0.045 & 1.589 & 645 & 0.069 & 0.010 & 0.049 & 0.090 & 0.151 & 1.373 & 870 \\
\hline General secondary & 0.195 & 0.024 & 0.147 & 0.243 & 0.125 & 1.643 & 416 & 0.023 & 0.007 & 0.009 & 0.036 & 0.307 & 1.051 & 515 \\
\hline Vocational secondary & 0.680 & 0.013 & 0.654 & 0.705 & 0.019 & 1.747 & 2,023 & 0.138 & 0.010 & 0.118 & 0.157 & 0.071 & 1.429 & 1,949 \\
\hline Post-secondary institute & 0.659 & 0.037 & 0.586 & 0.733 & 0.057 & 1.270 & 187 & 0.317 & 0.043 & 0.231 & 0.402 & 0.137 & 1.331 & 165 \\
\hline University and above & 0.570 & 0.018 & 0.535 & 0.604 & 0.031 & 1.450 & 1,014 & 0.295 & 0.018 & 0.261 & 0.330 & 0.059 & 1.471 & 981 \\
\hline \multicolumn{15}{|l|}{ Wealth quintile } \\
\hline Lowest & 0.669 & 0.020 & 0.630 & 0.707 & 0.029 & 1.702 & 827 & 0.129 & 0.015 & 0.100 & 0.158 & 0.114 & 1.921 & 1,017 \\
\hline Second & 0.666 & 0.018 & 0.630 & 0.702 & 0.028 & 1.685 & 950 & 0.135 & 0.013 & 0.110 & 0.160 & 0.095 & 1.488 & 1,141 \\
\hline Middle & 0.640 & 0.018 & 0.606 & 0.675 & 0.027 & 1.366 & 983 & 0.123 & 0.012 & 0.099 & 0.147 & 0.099 & 1.378 & 1,163 \\
\hline Fourth & 0.599 & 0.018 & 0.564 & 0.634 & 0.030 & 1.515 & 1,080 & 0.139 & 0.013 & 0.113 & 0.165 & 0.095 & 1.482 & 1,187 \\
\hline Highest & 0.561 & 0.016 & 0.530 & 0.593 & 0.029 & 1.395 & 1,220 & 0.192 & 0.014 & 0.165 & 0.219 & 0.072 & 1.487 & 1,316 \\
\hline
\end{tabular}


Table S3 Indicator: Unemployment rate (broad definition)

\begin{tabular}{|c|c|c|c|c|c|c|c|c|c|c|c|c|c|c|}
\hline \multirow[b]{3}{*}{ Characteristic } & \multirow[b]{3}{*}{ Estimate } & \multirow[b]{3}{*}{ SE } & \multicolumn{3}{|c|}{ Male } & \multirow{3}{*}{$\begin{array}{c}\text { Design } \\
\text { effect }\end{array}$} & \multirow[b]{3}{*}{ Count } & \multirow[b]{3}{*}{ Estimate } & \multirow[b]{3}{*}{ SE } & \multicolumn{2}{|c|}{ Female } & \multirow{3}{*}{$\begin{array}{l}\text { CV } \\
(\%)\end{array}$} & \multirow{3}{*}{$\begin{array}{l}\text { Design } \\
\text { effect }\end{array}$} & \multirow[b]{3}{*}{ Count } \\
\hline & & & $95 \%$ & C.I & CV & & & & & $95 \%$ & C.I & & & \\
\hline & & & Lower & Upper & $(\%)$ & & & & & Lower & Upper & & & \\
\hline \multicolumn{15}{|l|}{ Urban-rural residence } \\
\hline Urban & 0.128 & 0.015 & 0.099 & 0.157 & 0.116 & 1.934 & 999 & 0.335 & 0.037 & 0.262 & 0.409 & 0.112 & 1.801 & 295 \\
\hline Rural & 0.113 & 0.009 & 0.094 & 0.131 & 0.083 & 1.798 & 1,925 & 0.460 & 0.028 & 0.405 & 0.515 & 0.061 & 1.582 & 580 \\
\hline Informal urban areas & 0.139 & 0.027 & 0.086 & 0.192 & 0.195 & 1.825 & 290 & 0.536 & 0.056 & 0.426 & 0.647 & 0.105 & 0.998 & 100 \\
\hline \multicolumn{15}{|l|}{ Region } \\
\hline Urban Governorates & 0.135 & 0.015 & 0.105 & 0.165 & 0.113 & 1.293 & 653 & 0.306 & 0.044 & 0.220 & 0.394 & 0.143 & 1.865 & 203 \\
\hline Urban Lower Egypt & 0.153 & 0.028 & 0.098 & 0.209 & 0.184 & 2.107 & 336 & 0.474 & 0.058 & 0.360 & 0.588 & 0.123 & 1.457 & 120 \\
\hline Rural Lower Egypt & 0.145 & 0.014 & 0.118 & 0.172 & 0.094 & 1.615 & 1,058 & 0.523 & 0.035 & 0.450 & 0.593 & 0.068 & 1.353 & 337 \\
\hline Urban Upper Egypt & 0.085 & 0.032 & 0.022 & 0.149 & 0.380 & 3.469 & 164 & 0.451 & 0.086 & 0.280 & 0.619 & 0.190 & 1.358 & 36 \\
\hline Rural Upper Egypt & 0.077 & 0.012 & 0.054 & 0.100 & 0.153 & 1.803 & 694 & 0.387 & 0.042 & 0.300 & 0.470 & 0.109 & 1.736 & 225 \\
\hline Frontier Governorates & 0.108 & 0.027 & 0.056 & 0.160 & 0.246 & 0.469 & 309 & 0.475 & 0.086 & 0.310 & 0.644 & 0.180 & 0.292 & 54 \\
\hline \multicolumn{15}{|l|}{ Age group } \\
\hline $13-17$ years & 0.114 & 0.022 & 0.070 & 0.158 & 0.197 & 1.056 & 226 & 0.339 & 0.085 & 0.170 & 0.506 & 0.252 & 1.273 & 36 \\
\hline $18-24$ years & 0.176 & 0.013 & 0.150 & 0.203 & 0.076 & 1.527 & 1,191 & 0.499 & 0.034 & 0.430 & 0.567 & 0.069 & 1.534 & 325 \\
\hline $25-29$ years & 0.102 & 0.012 & 0.078 & 0.126 & 0.120 & 1.798 & 1,031 & 0.418 & 0.032 & 0.350 & 0.482 & 0.077 & 1.183 & 326 \\
\hline $30-35$ years & 0.054 & 0.011 & 0.032 & 0.075 & 0.202 & 1.779 & 766 & 0.345 & 0.031 & 0.280 & 0.406 & 0.091 & 0.990 & 288 \\
\hline \multicolumn{15}{|l|}{ Education level } \\
\hline Illiterate & 0.055 & 0.019 & 0.018 & 0.092 & 0.344 & 1.563 & 240 & 0.141 & 0.041 & 0.060 & 0.221 & 0.288 & 0.852 & 71 \\
\hline Read and write & 0.019 & 0.019 & -0.017 & 0.056 & 0.962 & 0.621 & 36 & 0.228 & 0.153 & -0.070 & 0.529 & 0.672 & 0.959 & 7 \\
\hline Primary & 0.081 & 0.018 & 0.046 & 0.116 & 0.220 & 1.691 & 401 & 0.329 & 0.070 & 0.190 & 0.466 & 0.211 & 1.262 & 72 \\
\hline Preparatory & 0.085 & 0.017 & 0.052 & 0.119 & 0.200 & 1.313 & 339 & 0.288 & 0.056 & 0.180 & 0.397 & 0.193 & 0.966 & 71 \\
\hline General secondary & 0.118 & 0.037 & 0.045 & 0.191 & 0.315 & 1.088 & 73 & 0.421 & 0.152 & 0.120 & 0.719 & 0.360 & 1.238 & 14 \\
\hline Vocational secondary & 0.120 & 0.011 & 0.098 & 0.143 & 0.095 & 1.808 & 1,420 & 0.538 & 0.035 & 0.470 & 0.606 & 0.064 & 1.440 & 350 \\
\hline Post-secondary institute & 0.159 & 0.034 & 0.092 & 0.227 & 0.216 & 1.143 & 120 & 0.493 & 0.087 & 0.320 & 0.665 & 0.177 & 1.423 & 50 \\
\hline University and above & 0.181 & 0.020 & 0.143 & 0.220 & 0.108 & 1.610 & 585 & 0.416 & 0.032 & 0.350 & 0.478 & 0.076 & 1.315 & 340 \\
\hline \multicolumn{15}{|l|}{ Wealth quintile } \\
\hline Lowest & 0.083 & 0.014 & 0.055 & 0.110 & 0.171 & 1.618 & 553 & 0.349 & 0.045 & 0.260 & 0.437 & 0.128 & 1.234 & 147 \\
\hline Second & 0.090 & 0.014 & 0.062 & 0.118 & 0.160 & 1.776 & 638 & 0.377 & 0.049 & 0.280 & 0.474 & 0.130 & 1.640 & 170 \\
\hline Middle & 0.146 & 0.017 & 0.113 & 0.178 & 0.114 & 1.404 & 651 & 0.395 & 0.049 & 0.300 & 0.492 & 0.124 & 1.366 & 157 \\
\hline Fourth & 0.132 & 0.016 & 0.101 & 0.163 & 0.119 & 1.411 & 671 & 0.537 & 0.042 & 0.450 & 0.620 & 0.078 & 1.173 & 200 \\
\hline Highest & 0.145 & 0.015 & 0.117 & 0.174 & 0.100 & 1.219 & 701 & 0.441 & 0.036 & 0.370 & 0.512 & 0.082 & 1.409 & 301 \\
\hline
\end{tabular}


Table S4 Indicator: Unemployment rate (market definition)

\begin{tabular}{|c|c|c|c|c|c|c|c|c|c|c|c|c|c|c|}
\hline \multirow[b]{3}{*}{ Characteristic } & \multirow[b]{3}{*}{ Estimate } & \multirow[b]{3}{*}{ SE } & \multicolumn{3}{|c|}{ Male } & \multirow{3}{*}{$\begin{array}{l}\text { Design } \\
\text { effect }\end{array}$} & \multirow[b]{3}{*}{ Count } & \multirow[b]{3}{*}{ Estimate } & \multirow[b]{3}{*}{ SE } & \multicolumn{2}{|c|}{ Female } & \multirow{3}{*}{$\begin{array}{l}\text { CV } \\
(\%)\end{array}$} & \multirow{3}{*}{$\begin{array}{c}\text { Design } \\
\text { effect }\end{array}$} & \multirow[b]{3}{*}{ Count } \\
\hline & & & \multicolumn{2}{|c|}{ 95\% C.I } & \multirow{2}{*}{$\begin{array}{l}\text { CV } \\
(\%)\end{array}$} & & & & & \multicolumn{2}{|c|}{ 95\% C.I } & & & \\
\hline & & & Lower & Upper & & & & & & Lower & Upper & & & \\
\hline \multicolumn{15}{|l|}{ Urban-rural residence } \\
\hline Urban & 0.098 & 0.013 & 0.072 & 0.124 & 0.134 & 1.842 & 962 & 0.249 & 0.036 & 0.179 & 0.319 & 0.143 & 1.713 & 261 \\
\hline Rural & 0.075 & 0.007 & 0.061 & 0.089 & 0.096 & 1.435 & 1,840 & 0.316 & 0.028 & 0.262 & 0.371 & 0.088 & 1.409 & 453 \\
\hline Informal urban areas & 0.054 & 0.015 & 0.024 & 0.083 & 0.279 & 1.190 & 265 & 0.338 & 0.068 & 0.205 & 0.472 & 0.201 & 1.126 & 71 \\
\hline \multicolumn{15}{|l|}{ Region } \\
\hline Urban Governorates & 0.103 & 0.013 & 0.078 & 0.129 & 0.126 & 1.139 & 627 & 0.221 & 0.042 & 0.140 & 0.304 & 0.192 & 1.886 & 176 \\
\hline Urban Lower Egypt & 0.094 & 0.025 & 0.046 & 0.142 & 0.262 & 2.282 & 312 & 0.309 & 0.063 & 0.190 & 0.433 & 0.203 & 1.497 & 92 \\
\hline Rural Lower Egypt & 0.097 & 0.011 & 0.076 & 0.118 & 0.109 & 1.286 & 1,000 & 0.382 & 0.038 & 0.310 & 0.457 & 0.100 & 1.265 & 256 \\
\hline Urban Upper Egypt & 0.040 & 0.023 & 0.000 & 0.086 & 0.579 & 3.438 & 156 & 0.342 & 0.088 & 0.170 & 0.514 & 0.257 & 1.297 & 31 \\
\hline Rural Upper Egypt & 0.051 & 0.009 & 0.034 & 0.068 & 0.172 & 1.418 & 674 & 0.246 & 0.039 & 0.170 & 0.322 & 0.158 & 1.517 & 184 \\
\hline Frontier Governorates & 0.073 & 0.023 & 0.027 & 0.119 & 0.323 & 0.500 & 298 & 0.395 & 0.100 & 0.200 & 0.593 & 0.254 & 0.362 & 46 \\
\hline \multicolumn{15}{|l|}{ Age group } \\
\hline 13-17 years & 0.042 & 0.015 & 0.011 & 0.072 & 0.369 & 1.146 & 209 & 0.083 & 0.042 & -0.000 & 0.166 & 0.510 & 0.660 & 26 \\
\hline $18-24$ years & 0.123 & 0.012 & 0.100 & 0.146 & 0.096 & 1.505 & 1,105 & 0.343 & 0.036 & 0.270 & 0.414 & 0.105 & 1.418 & 240 \\
\hline $25-29$ years & 0.070 & 0.010 & 0.051 & 0.089 & 0.140 & 1.556 & 996 & 0.327 & 0.033 & 0.260 & 0.392 & 0.100 & 1.157 & 280 \\
\hline $30-35$ years & 0.037 & 0.008 & 0.021 & 0.053 & 0.222 & 1.411 & 757 & 0.226 & 0.029 & 0.160 & 0.277 & 0.130 & 0.915 & 239 \\
\hline \multicolumn{15}{|l|}{ Education level } \\
\hline Illiterate & 0.024 & 0.011 & 0.001 & 0.046 & 0.479 & 1.211 & 230 & 0.030 & 0.021 & -0.010 & 0.071 & 0.721 & 0.858 & 61 \\
\hline Read and write & 0.019 & 0.019 & 0.000 & 0.056 & 0.962 & 0.616 & 36 & 0.000 & 0.000 & 0.000 & 0.000 & 0.000 & 0.000 & 5 \\
\hline Primary & 0.053 & 0.016 & 0.022 & 0.084 & 0.298 & 1.901 & 389 & 0.208 & 0.069 & 0.070 & 0.344 & 0.334 & 1.418 & 59 \\
\hline Preparatory & 0.056 & 0.014 & 0.028 & 0.085 & 0.258 & 1.337 & 328 & 0.113 & 0.039 & 0.040 & 0.190 & 0.347 & 0.783 & 56 \\
\hline General secondary & 0.075 & 0.031 & 0.014 & 0.136 & 0.412 & 1.069 & 69 & 0.241 & 0.157 & -0.070 & 0.550 & 0.654 & 1.345 & 12 \\
\hline Vocational secondary & 0.073 & 0.009 & 0.056 & 0.090 & 0.117 & 1.501 & 1,348 & 0.377 & 0.037 & 0.300 & 0.451 & 0.099 & 1.315 & 254 \\
\hline Post-secondary institute & 0.116 & 0.031 & 0.056 & 0.176 & 0.263 & 1.110 & 113 & 0.466 & 0.091 & 0.290 & 0.645 & 0.196 & 1.466 & 47 \\
\hline University and above & 0.143 & 0.018 & 0.108 & 0.179 & 0.125 & 1.564 & 554 & 0.309 & 0.033 & 0.250 & 0.374 & 0.106 & 1.343 & 291 \\
\hline \multicolumn{15}{|l|}{ Wealth quintile } \\
\hline Lowest & 0.061 & 0.013 & 0.036 & 0.087 & 0.211 & 1.734 & 537 & 0.226 & 0.043 & 0.140 & 0.310 & 0.188 & 1.219 & 120 \\
\hline Second & 0.054 & 0.011 & 0.033 & 0.076 & 0.204 & 1.612 & 613 & 0.244 & 0.045 & 0.160 & 0.332 & 0.184 & 1.427 & 142 \\
\hline Middle & 0.090 & 0.013 & 0.064 & 0.117 & 0.148 & 1.285 & 612 & 0.273 & 0.051 & 0.170 & 0.373 & 0.186 & 1.462 & 130 \\
\hline Fourth & 0.088 & 0.014 & 0.061 & 0.114 & 0.155 & 1.420 & 636 & 0.412 & 0.047 & 0.320 & 0.505 & 0.115 & 1.201 & 152 \\
\hline Highest & 0.106 & 0.013 & 0.081 & 0.130 & 0.119 & 1.118 & 669 & 0.301 & 0.038 & 0.230 & 0.375 & 0.125 & 1.421 & 241 \\
\hline
\end{tabular}




\begin{tabular}{|c|c|c|c|c|c|c|c|c|c|c|c|c|c|c|}
\hline \multirow[b]{3}{*}{ Characteristic } & \multirow[b]{3}{*}{ Estimate } & \multirow[b]{3}{*}{ SE } & \multicolumn{3}{|c|}{ Male } & \multirow{3}{*}{$\begin{array}{c}\text { Design } \\
\text { effect }\end{array}$} & \multirow[b]{3}{*}{ Count } & \multirow[b]{3}{*}{ Estimate } & \multirow[b]{3}{*}{ SE } & \multicolumn{2}{|c|}{ Female } & \multirow{3}{*}{$\begin{array}{l}\text { CV } \\
(\%)\end{array}$} & \multirow{3}{*}{$\begin{array}{c}\text { Design } \\
\text { effect }\end{array}$} & \multirow[b]{3}{*}{ Count } \\
\hline & & & \multicolumn{2}{|c|}{ 95\% C.I } & \multirow{2}{*}{$\begin{array}{l}\text { CV } \\
(\%)\end{array}$} & & & & & \multicolumn{2}{|c|}{ 95\% C.I } & & & \\
\hline & & & Lower & Upper & & & & & & Lower & Upper & & & \\
\hline \multicolumn{15}{|l|}{ Urban-rural residence } \\
\hline Urban & 0.902 & 0.013 & 0.876 & 0.928 & 0.015 & 1.842 & 962 & 0.751 & 0.036 & 0.681 & 0.821 & 0.048 & 1.713 & 261 \\
\hline Rural & 0.925 & 0.007 & 0.911 & 0.939 & 0.008 & 1.435 & 1,840 & 0.684 & 0.028 & 0.629 & 0.738 & 0.041 & 1.409 & 453 \\
\hline Informal urban areas & 0.946 & 0.015 & 0.917 & 0.976 & 0.016 & 1.190 & 265 & 0.662 & 0.068 & 0.528 & 0.795 & 0.103 & 1.126 & 71 \\
\hline \multicolumn{15}{|l|}{ Region } \\
\hline Urban Governorates & 0.897 & 0.013 & 0.871 & 0.922 & 0.015 & 1.139 & 627 & 0.779 & 0.042 & 0.700 & 0.862 & 0.054 & 1.886 & 176 \\
\hline Urban Lower Egypt & 0.906 & 0.025 & 0.858 & 0.954 & 0.027 & 2.282 & 312 & 0.690 & 0.063 & 0.570 & 0.814 & 0.091 & 1.497 & 92 \\
\hline Rural Lower Egypt & 0.903 & 0.011 & 0.882 & 0.924 & 0.012 & 1.286 & 1,000 & 0.618 & 0.038 & 0.540 & 0.693 & 0.062 & 1.265 & 256 \\
\hline Urban Upper Egypt & 0.960 & 0.023 & 0.914 & 1.006 & 0.024 & 3.438 & 156 & 0.658 & 0.088 & 0.490 & 0.831 & 0.133 & 1.297 & 31 \\
\hline Rural Upper Egypt & 0.949 & 0.009 & 0.932 & 0.966 & 0.009 & 1.418 & 674 & 0.754 & 0.039 & 0.680 & 0.831 & 0.052 & 1.517 & 184 \\
\hline Frontier Governorates & 0.927 & 0.023 & 0.881 & 0.973 & 0.025 & 0.500 & 298 & 0.604 & 0.100 & 0.410 & 0.802 & 0.166 & 0.362 & 46 \\
\hline \multicolumn{15}{|l|}{ Age group } \\
\hline 13-17 years & 0.958 & 0.015 & 0.928 & 0.989 & 0.016 & 1.146 & 209 & 0.917 & 0.042 & 0.830 & 1.000 & 0.046 & 0.66 & 26 \\
\hline $18-24$ years & 0.877 & 0.012 & 0.854 & 0.900 & 0.014 & 1.505 & 1,105 & 0.657 & 0.036 & 0.590 & 0.728 & 0.055 & 1.418 & 240 \\
\hline $25-29$ years & 0.930 & 0.010 & 0.911 & 0.949 & 0.011 & 1.556 & 996 & 0.672 & 0.033 & 0.610 & 0.737 & 0.049 & 1.157 & 280 \\
\hline $30-35$ years & 0.963 & 0.008 & 0.947 & 0.979 & 0.009 & 1.411 & 757 & 0.779 & 0.029 & 0.720 & 0.836 & 0.037 & 0.915 & 239 \\
\hline \multicolumn{15}{|l|}{ Education level } \\
\hline Illiterate & 0.976 & 0.011 & 0.954 & 0.999 & 0.012 & 1.211 & 230 & 0.971 & 0.021 & 0.930 & 1.012 & 0.022 & 0.858 & 61 \\
\hline Read and write & 0.981 & 0.019 & 0.944 & 1.017 & 0.019 & 0.616 & 36 & 0.000 & 0.000 & 0.000 & 0.000 & 0.000 & 0.000 & 5 \\
\hline Primary & 0.947 & 0.016 & 0.916 & 0.978 & 0.017 & 1.901 & 389 & 0.792 & 0.069 & 0.660 & 0.929 & 0.088 & 1.418 & 59 \\
\hline Preparatory & 0.944 & 0.014 & 0.915 & 0.972 & 0.015 & 1.337 & 328 & 0.887 & 0.039 & 0.810 & 0.964 & 0.044 & 0.783 & 56 \\
\hline General secondary & 0.925 & 0.031 & 0.864 & 0.986 & 0.034 & 1.069 & 69 & 0.759 & 0.157 & 0.450 & 1.069 & 0.207 & 1.345 & 12 \\
\hline Vocational secondary & 0.927 & 0.009 & 0.910 & 0.944 & 0.009 & 1.501 & 1,348 & 0.623 & 0.037 & 0.550 & 0.697 & 0.060 & 1.315 & 254 \\
\hline Post-secondary institute & 0.884 & 0.031 & 0.824 & 0.944 & 0.035 & 1.110 & 113 & 0.534 & 0.091 & 0.350 & 0.713 & 0.171 & 1.466 & 47 \\
\hline University and above & 0.857 & 0.018 & 0.821 & 0.892 & 0.021 & 1.564 & 554 & 0.691 & 0.033 & 0.630 & 0.755 & 0.048 & 1.343 & 291 \\
\hline \multicolumn{15}{|l|}{ Wealth quintile } \\
\hline Lowest & 0.939 & 0.013 & 0.913 & 0.964 & 0.014 & 1.734 & 537 & 0.774 & 0.043 & 0.690 & 0.858 & 0.055 & 1.219 & 120 \\
\hline Second & 0.946 & 0.011 & 0.924 & 0.967 & 0.012 & 1.612 & 613 & 0.756 & 0.045 & 0.670 & 0.844 & 0.059 & 1.427 & 142 \\
\hline Middle & 0.910 & 0.013 & 0.883 & 0.936 & 0.015 & 1.285 & 612 & 0.727 & 0.051 & 0.630 & 0.827 & 0.070 & 1.462 & 130 \\
\hline Fourth & 0.912 & 0.014 & 0.886 & 0.939 & 0.015 & 1.420 & 636 & 0.587 & 0.047 & 0.490 & 0.681 & 0.081 & 1.201 & 152 \\
\hline Highest & 0.894 & 0.013 & 0.870 & 0.919 & 0.014 & 1.118 & 669 & 0.698 & 0.038 & 0.620 & 0.773 & 0.054 & 1.421 & 241 \\
\hline
\end{tabular}




\begin{tabular}{|c|c|c|c|c|c|c|c|c|c|c|c|c|c|c|}
\hline \multirow[b]{3}{*}{ Characteristic } & \multirow[b]{3}{*}{ Estimate } & \multirow[b]{3}{*}{ SE } & \multicolumn{3}{|c|}{ Male } & \multirow{3}{*}{$\begin{array}{l}\text { Design } \\
\text { effect }\end{array}$} & \multirow[b]{3}{*}{ Count } & \multirow[b]{3}{*}{ Estimate } & \multirow[b]{3}{*}{ SE } & \multirow{2}{*}{\multicolumn{2}{|c|}{$\begin{array}{l}\text { Female } \\
5 \% \text { C.I }\end{array}$}} & \multirow{3}{*}{$\begin{array}{l}\text { CV } \\
(\%)\end{array}$} & \multirow{3}{*}{$\begin{array}{c}\text { Design } \\
\text { effect }\end{array}$} & \multirow[b]{3}{*}{ Count } \\
\hline & & & \multicolumn{2}{|c|}{ 95\% C.I } & \multirow{2}{*}{$\begin{array}{l}\text { CV } \\
(\%)\end{array}$} & & & & & & & & & \\
\hline & & & Lower & Upper & & & & & & Lower & Upper & & & \\
\hline Urban-rural residence & & & & & & & & & & & & & & \\
\hline Urban & 50.214 & 0.846 & 48.552 & 51.877 & 0.017 & 2.242 & 872 & 46.545 & 1.259 & 44.070 & 49.020 & 0.027 & 1.504 & 194 \\
\hline Rural & 45.644 & 0.779 & 44.112 & 47.175 & 0.017 & 3.298 & 1,706 & 39.732 & 1.171 & 37.429 & 42.034 & 0.029 & 1.160 & 310 \\
\hline Informal urban areas & 49.151 & 1.510 & 46.184 & 52.119 & 0.031 & 2.218 & 250 & 45.942 & 2.537 & 40.956 & 50.928 & 0.055 & 1.065 & 47 \\
\hline Region & & & & & & & & & & & & & & \\
\hline Urban Governorates & 52.089 & 0.791 & 50.534 & 53.644 & 0.015 & 1.219 & 568 & 48.326 & 1.437 & 45.502 & 51.149 & 0.030 & 1.369 & 136 \\
\hline Rural Lower Egypt & 49.517 & 1.034 & 47.485 & 51.549 & 0.021 & 3.217 & 910 & 42.673 & 1.592 & 39.544 & 45.801 & 0.037 & 0.999 & 155 \\
\hline Urban Upper Egypt & 43.239 & 1.406 & 40.476 & 46.002 & 0.033 & 2.377 & 149 & 40.441 & 1.849 & 36.807 & 44.076 & 0.046 & 1.038 & 21 \\
\hline Rural Upper Egypt & 41.416 & 0.961 & 39.528 & 43.305 & 0.023 & 2.389 & 637 & 37.085 & 1.710 & 33.724 & 40.446 & 0.046 & 1.361 & 143 \\
\hline Frontier Governorates & 49.213 & 1.810 & 45.655 & 52.771 & 0.037 & 0.875 & 276 & 39.825 & 2.145 & 35.610 & 44.041 & 0.054 & 0.216 & 34 \\
\hline Age group & & & & & & & & & & & & & & \\
\hline $13-17$ years & 45.094 & 1.852 & 41.454 & 48.733 & 0.041 & 1.520 & 201 & 39.146 & 4.376 & 30.547 & 47.746 & 0.112 & 1.340 & 23 \\
\hline $18-24$ years & 46.560 & 0.815 & 44.958 & 48.162 & 0.018 & 2.035 & 961 & 44.622 & 1.472 & 41.729 & 47.516 & 0.033 & 1.145 & 153 \\
\hline $25-29$ years & 47.740 & 0.714 & 46.337 & 49.143 & 0.015 & 1.727 & 933 & 41.899 & 1.291 & 39.361 & 44.436 & 0.031 & 1.071 & 189 \\
\hline $30-35$ years & 48.312 & 0.778 & 46.783 & 49.842 & 0.016 & 1.540 & 733 & 42.326 & 1.218 & 39.933 & 44.719 & 0.029 & 0.858 & 186 \\
\hline Illiterate & 47.036 & 1.926 & 43.250 & 50.823 & 0.041 & 2.389 & 223 & 42.941 & 3.098 & 36.853 & 49.029 & 0.072 & 1.057 & 59 \\
\hline Read and write & 51.615 & 2.365 & 46.967 & 56.263 & 0.046 & 1.231 & 35 & 47.797 & 6.326 & 35.363 & 60.230 & 0.132 & 0.840 & 6 \\
\hline Primary & 48.446 & 1.135 & 46.216 & 50.676 & 0.023 & 1.574 & 373 & 46.628 & 3.314 & 40.114 & 53.141 & 0.071 & 1.084 & 47 \\
\hline Preparatory & 48.858 & 1.465 & 45.979 & 51.737 & 0.030 & 1.763 & 310 & 43.795 & 2.983 & 37.932 & 49.657 & 0.068 & 1.202 & 47 \\
\hline General secondary & 42.793 & 2.253 & 38.364 & 47.221 & 0.053 & 1.296 & 63 & 36.877 & 5.795 & 25.488 & 48.266 & 0.157 & 0.785 & 10 \\
\hline Vocational secondary & 46.759 & 0.777 & 45.232 & 48.286 & 0.017 & 2.351 & 1,246 & 42.383 & 1.596 & 39.246 & 45.519 & 0.038 & 1.227 & 153 \\
\hline Post-secondary institute & 48.466 & 2.392 & 43.765 & 53.168 & 0.049 & 1.501 & 97 & 46.423 & 3.099 & 40.333 & 52.513 & 0.067 & 0.977 & 27 \\
\hline University and above & 47.083 & 0.786 & 45.537 & 48.628 & 0.017 & 1.532 & 481 & 41.570 & 1.136 & 39.337 & 43.804 & 0.027 & 1.406 & 202 \\
\hline Wealth quintile & & & & & & & & & & & & & & \\
\hline Lowest & 46.040 & 1.080 & 43.918 & 48.162 & 0.023 & 2.003 & 509 & 40.933 & 1.928 & 37.144 & 44.721 & 0.047 & 1.005 & 91 \\
\hline Second & 45.870 & 1.073 & 43.761 & 47.979 & 0.023 & 2.412 & 581 & 47.352 & 1.751 & 43.912 & 50.793 & 0.037 & 1.020 & 107 \\
\hline Middle & 47.372 & 1.065 & 45.279 & 49.466 & 0.022 & 1.983 & 557 & 41.202 & 1.919 & 37.431 & 44.974 & 0.047 & 0.953 & 95 \\
\hline Fourth & 48.482 & 1.065 & 46.389 & 50.575 & 0.022 & 1.999 & 584 & 44.240 & 1.854 & 40.596 & 47.883 & 0.042 & 1.035 & 92 \\
\hline Highest & 48.820 & 0.920 & 47.011 & 50.629 & 0.019 & 1.735 & 597 & 40.913 & 1.301 & 38.356 & 43.470 & 0.032 & 1.271 & 166 \\
\hline
\end{tabular}


Table S7 Indicator: Average total monthly earnings for wage and salaried workers in reference 3 months

\begin{tabular}{|c|c|c|c|c|c|c|c|c|c|c|c|c|c|c|}
\hline \multirow[b]{2}{*}{ Characteristic } & \multirow[b]{2}{*}{ Estimate } & \multirow[b]{2}{*}{ SE } & \multicolumn{2}{|c|}{$\begin{array}{c}\text { Male } \\
95 \% \text { C.I }\end{array}$} & \multirow{2}{*}{$\begin{array}{l}\text { CV } \\
(\%)\end{array}$} & \multirow{2}{*}{$\begin{array}{l}\text { Design } \\
\text { effect }\end{array}$} & \multirow[b]{2}{*}{ Count } & \multirow[b]{2}{*}{ Estimate } & \multirow[b]{2}{*}{ SE } & \multicolumn{2}{|c|}{$\begin{array}{l}\text { Female } \\
95 \% \text { C.I }\end{array}$} & \multirow{2}{*}{$\begin{array}{l}\text { CV } \\
\text { (\%) }\end{array}$} & \multirow{2}{*}{$\begin{array}{l}\text { Design } \\
\text { effect }\end{array}$} & \multirow[b]{2}{*}{ Count } \\
\hline & & & Lower & Upper & & & & & & Lower & Upper & & & \\
\hline \multicolumn{15}{|l|}{ Urban-rural residence } \\
\hline Urban & 1,883.615 & 246.409 & 1,399.339 & $2,367.892$ & 0.131 & 2.490 & 873 & $1,321.103$ & 231.875 & 865.392 & $1,776.814$ & 0.176 & 0.987 & 195 \\
\hline Rural & $1,633.386$ & 166.001 & $1,307.138$ & $1,959.634$ & 0.102 & 0.980 & 1,706 & $1,177.544$ & 340.229 & 508.880 & $1,846.208$ & 0.289 & 2.312 & 311 \\
\hline Informal urban areas & $2,295.441$ & 617.819 & $1,081.220$ & $3,509.661$ & 0.269 & 1.032 & 250 & $2,599.616$ & $1,026.037$ & 583.113 & $4,616.120$ & 0.395 & 0.659 & 47 \\
\hline \multicolumn{15}{|l|}{ Region } \\
\hline Urban Governorates & $2,164.423$ & 365.321 & $1,446.446$ & $2,882.401$ & 0.169 & 2.672 & 568 & $1,196.708$ & 214.938 & 774.284 & 1,619.132 & 0.180 & 0.645 & 137 \\
\hline Urban Lower Egypt & $2,062.271$ & 498.237 & $1,083.069$ & $3,041.473$ & 0.242 & 0.947 & 288 & $1,384.443$ & 460.372 & 479.660 & $2,289.227$ & 0.333 & 0.689 & 62 \\
\hline Rural Lower Egypt & $1,969.302$ & 284.635 & $1,409.900$ & $2,528.704$ & 0.145 & 0.854 & 910 & $1,018.681$ & 161.677 & 700.932 & $1,336.431$ & 0.159 & 0.762 & 155 \\
\hline Urban Upper Egypt & $1,548.288$ & 307.387 & 944.169 & $2,152.406$ & 0.199 & 1.209 & 149 & $3,594.129$ & $1,676.413$ & 299.418 & $6,888.840$ & 0.466 & 1.168 & 21 \\
\hline Rural Upper Egypt & $1,304.669$ & 161.037 & 988.177 & $1,621.161$ & 0.123 & 1.682 & 637 & $1,328.487$ & 632.485 & 85.444 & $2,571.530$ & 0.476 & 2.562 & 144 \\
\hline Frontier Governorates & 843.510 & 96.529 & 653.799 & $1,033.221$ & 0.114 & 0.485 & 277 & $1,748.106$ & 788.772 & 197.906 & $3,298.305$ & 0.451 & 0.224 & 34 \\
\hline \multicolumn{15}{|l|}{ Age group } \\
\hline $13-17$ years & $1,529.981$ & 369.946 & 802.914 & $2,257.048$ & 0.242 & 1.067 & 201 & 327.742 & 119.323 & 93.234 & 562.251 & 0.364 & 1.063 & 23 \\
\hline $18-24$ years & $1,519.544$ & 173.903 & $1,177.766$ & $1,861.322$ & 0.114 & 1.809 & 961 & $1,129.509$ & 276.774 & 585.557 & $1,673.461$ & 0.245 & 1.026 & 153 \\
\hline $25-29$ years & $1,944.762$ & 265.859 & $1,422.260$ & $2,467.264$ & 0.137 & 0.793 & 934 & $1,350.414$ & 353.150 & 656.356 & $2,044.472$ & 0.262 & 1.436 & 191 \\
\hline $30-35$ years & $1,927.479$ & 215.571 & $1,503.811$ & $2,351.147$ & 0.112 & 0.994 & 733 & $1,717.583$ & 376.264 & 978.099 & $2,457.067$ & 0.219 & 0.889 & 186 \\
\hline \multicolumn{15}{|l|}{ Education level } \\
\hline Illiterate & $1,485.489$ & 364.640 & 768.850 & $2,202.130$ & 0.245 & 1.895 & 223 & 153.042 & 56.561 & 41.880 & 264.204 & 0.370 & 0.976 & 59 \\
\hline Read and write & $1,533.811$ & 907.400 & -249.530 & $3,317.146$ & 0.592 & 1.560 & 35 & 322.278 & 186.390 & -44.040 & 688.597 & 0.578 & 1.493 & 6 \\
\hline Primary & $1,546.113$ & 263.080 & $1,029.070$ & $2,063.161$ & 0.170 & 1.141 & 373 & $1,376.157$ & 706.974 & -13.281 & $2,765.596$ & 0.514 & 0.581 & 47 \\
\hline Preparatory & $1,679.740$ & 245.760 & $1,196.750$ & $2,162.734$ & 0.146 & 1.098 & 311 & 550.481 & 259.579 & 40.322 & $1,060.640$ & 0.472 & 0.817 & 48 \\
\hline General secondary & $2,094.484$ & 768.190 & 584.734 & $3,604.235$ & 0.367 & 1.235 & 63 & 672.053 & 255.766 & 169.387 & $1,174.719$ & 0.381 & 0.624 & 10 \\
\hline Vocational secondary & $1,411.793$ & 102.220 & $1,210.903$ & $1,612.684$ & 0.072 & 1.130 & 1,246 & 873.683 & 203.455 & 473.826 & $1,273.539$ & 0.233 & 1.695 & 153 \\
\hline Post-secondary institute & $4,766.373$ & $2,137.400$ & 565.671 & $8,967.075$ & 0.448 & 0.724 & 97 & $2,918.650$ & $1,311.253$ & 341.601 & $5,495.699$ & 0.449 & 0.779 & 27 \\
\hline University and above & $2,316.377$ & 320.880 & $1,685.744$ & $2,947.011$ & 0.139 & 1.201 & 481 & $2,067.404$ & 512.072 & $1,061.012$ & $3,073.796$ & 0.248 & 2.052 & 203 \\
\hline \multicolumn{15}{|l|}{ Wealth quintile } \\
\hline Lowest & $1,418.002$ & 192.880 & $1,038.930$ & $1,797.075$ & 0.136 & 1.882 & 509 & 735.106 & 292.625 & 160.000 & $1,310.212$ & 0.398 & 0.598 & 91 \\
\hline Second & $1,431.224$ & 189.442 & $1,058.908$ & $1,803.540$ & 0.132 & 2.095 & 582 & 705.502 & 109.169 & 490.948 & 920.056 & 0.155 & 1.175 & 107 \\
\hline Middle & $1,731.824$ & 250.927 & $1,238.669$ & $2,224.978$ & 0.145 & 0.987 & 557 & $1,323.577$ & 441.148 & 456.575 & $2,190.579$ & 0.333 & 0.790 & 96 \\
\hline Fourth & $1,796.184$ & 234.905 & $1,334.518$ & $2,257.849$ & 0.131 & 1.376 & 584 & $1,609.883$ & 670.131 & 292.852 & $2,926.914$ & 0.416 & 1.196 & 92 \\
\hline Highest & $2,444.688$ & 435.689 & $1,588.415$ & $3,300.962$ & 0.178 & 0.787 & 597 & $1,997.641$ & 397.301 & $1,216.812$ & $2,778.470$ & 0.199 & 1.376 & 167 \\
\hline
\end{tabular}


Table S8 Indicator: Percentage of wage workers

\begin{tabular}{|c|c|c|c|c|c|c|c|c|c|c|c|c|c|c|}
\hline \multirow[b]{3}{*}{ Characteristic } & \multirow[b]{3}{*}{ Estimate } & \multirow[b]{3}{*}{ SE } & \multirow{2}{*}{\multicolumn{2}{|c|}{$\begin{array}{r}\text { Male } \\
95 \% \text { C.I }\end{array}$}} & \multirow{3}{*}{$\begin{array}{l}\text { CV } \\
(\%)\end{array}$} & \multirow{3}{*}{$\begin{array}{l}\text { Design } \\
\text { effect }\end{array}$} & \multirow[b]{3}{*}{ Count } & \multirow[b]{3}{*}{ Estimate } & \multirow[b]{3}{*}{ SE } & \multirow{2}{*}{\multicolumn{2}{|c|}{$\begin{array}{l}\text { Female } \\
95 \% \text { C.I }\end{array}$}} & \multirow{3}{*}{$\begin{array}{l}\text { CV } \\
(\%)\end{array}$} & \multirow{3}{*}{$\begin{array}{c}\text { Design } \\
\text { effect }\end{array}$} & \multirow[b]{3}{*}{ Count } \\
\hline & & & & & & & & & & & & & & \\
\hline & & & Lower & Upper & & & & & & Lower & Upper & & & \\
\hline \multicolumn{15}{|l|}{ Urban-rural residence } \\
\hline Urban & 0.817 & 0.016 & 0.786 & 0.848 & 0.020 & 1.445 & 871 & 0.910 & 0.026 & 0.860 & 0.960 & 0.028 & 1.500 & 195 \\
\hline Rural & 0.752 & 0.014 & 0.724 & 0.779 & 0.019 & 1.916 & 1,706 & 0.556 & 0.040 & 0.477 & 0.635 & 0.072 & 1.760 & 310 \\
\hline Informal urban areas & 0.722 & 0.038 & 0.648 & 0.795 & 0.052 & 1.781 & 250 & 0.803 & 0.074 & 0.658 & 0.948 & 0.092 & 1.234 & 47 \\
\hline \multicolumn{15}{|l|}{ Region } \\
\hline Urban Governorates & 0.825 & 0.019 & 0.787 & 0.863 & 0.023 & 1.440 & 567 & 0.906 & 0.033 & 0.841 & 0.971 & 0.036 & 1.791 & 137 \\
\hline Urban Lower Egypt & 0.711 & 0.034 & 0.643 & 0.779 & 0.048 & 1.661 & 288 & 0.893 & 0.042 & 0.810 & 0.975 & 0.047 & 1.026 & 62 \\
\hline Rural Lower Egypt & 0.726 & 0.020 & 0.687 & 0.765 & 0.027 & 1.767 & 910 & 0.712 & 0.043 & 0.627 & 0.797 & 0.061 & 1.155 & 155 \\
\hline Urban Upper Egypt & 0.828 & 0.033 & 0.764 & 0.892 & 0.039 & 1.743 & 149 & 0.825 & 0.084 & 0.660 & 0.990 & 0.102 & 1.218 & 21 \\
\hline Rural Upper Egypt & 0.785 & 0.020 & 0.747 & 0.824 & 0.025 & 1.946 & 637 & 0.415 & 0.056 & 0.305 & 0.525 & 0.135 & 1.799 & 143 \\
\hline Frontier Governorates & 0.679 & 0.066 & 0.550 & 0.809 & 0.097 & 1.126 & 276 & 0.743 & 0.138 & 0.472 & 1.014 & 0.186 & 0.514 & 34 \\
\hline \multicolumn{15}{|l|}{ Age group } \\
\hline $13-17$ years & 0.693 & 0.036 & 0.623 & 0.764 & 0.052 & 1.125 & 201 & 0.438 & 0.120 & 0.202 & 0.673 & 0.274 & 1.490 & 23 \\
\hline $18-24$ years & 0.768 & 0.017 & 0.734 & 0.802 & 0.023 & 1.714 & 961 & 0.717 & 0.047 & 0.624 & 0.810 & 0.066 & 1.763 & 153 \\
\hline $25-29$ years & 0.793 & 0.017 & 0.759 & 0.827 & 0.022 & 1.788 & 933 & 0.754 & 0.036 & 0.682 & 0.825 & 0.048 & 1.121 & 190 \\
\hline $30-35$ years & 0.754 & 0.019 & 0.717 & 0.791 & 0.025 & 1.374 & 732 & 0.701 & 0.038 & 0.626 & 0.777 & 0.055 & 1.040 & 186 \\
\hline \multicolumn{15}{|l|}{ Education level } \\
\hline Illiterate & 0.770 & 0.036 & 0.699 & 0.841 & 0.047 & 1.580 & 223 & 0.258 & 0.073 & 0.115 & 0.402 & 0.283 & 1.476 & 59 \\
\hline Read and write & 0.679 & 0.094 & 0.494 & 0.863 & 0.138 & 1.338 & 35 & 0.478 & 0.247 & -0.007 & 0.962 & 0.516 & 1.336 & 5 \\
\hline Primary & 0.725 & 0.027 & 0.672 & 0.778 & 0.037 & 1.315 & 372 & 0.615 & 0.084 & 0.449 & 0.781 & 0.137 & 1.148 & 47 \\
\hline Preparatory & 0.767 & 0.028 & 0.713 & 0.822 & 0.036 & 1.367 & 310 & 0.565 & 0.089 & 0.389 & 0.741 & 0.158 & 1.464 & 48 \\
\hline General secondary & 0.754 & 0.062 & 0.633 & 0.876 & 0.082 & 1.463 & 63 & 0.663 & 0.157 & 0.355 & 0.971 & 0.236 & 0.824 & 10 \\
\hline Vocational secondary & 0.769 & 0.016 & 0.738 & 0.799 & 0.020 & 1.739 & 1,246 & 0.678 & 0.046 & 0.587 & 0.769 & 0.069 & 1.350 & 153 \\
\hline Post-secondary institute & 0.817 & 0.043 & 0.732 & 0.902 & 0.053 & 1.351 & 97 & 0.871 & 0.063 & 0.748 & 0.995 & 0.072 & 0.818 & 27 \\
\hline University and above & 0.795 & 0.023 & 0.750 & 0.840 & 0.029 & 1.627 & 481 & 0.908 & 0.028 & 0.853 & 0.962 & 0.031 & 1.697 & 203 \\
\hline \multicolumn{15}{|l|}{ Wealth quintile } \\
\hline Lowest & 0.814 & 0.019 & 0.776 & 0.851 & 0.023 & 1.333 & 509 & 0.489 & 0.073 & 0.345 & 0.634 & 0.150 & 1.951 & 91 \\
\hline Second & 0.770 & 0.021 & 0.728 & 0.812 & 0.028 & 1.611 & 581 & 0.680 & 0.053 & 0.576 & 0.783 & 0.078 & 1.256 & 107 \\
\hline Middle & 0.727 & 0.023 & 0.681 & 0.773 & 0.032 & 1.484 & 556 & 0.594 & 0.061 & 0.474 & 0.714 & 0.103 & 1.260 & 96 \\
\hline Fourth & 0.777 & 0.023 & 0.732 & 0.821 & 0.029 & 1.665 & 584 & 0.822 & 0.045 & 0.732 & 0.911 & 0.055 & 1.069 & 91 \\
\hline Highest & 0.753 & 0.021 & 0.710 & 0.795 & 0.029 & 1.482 & 597 & 0.872 & 0.032 & 0.809 & 0.935 & 0.037 & 1.360 & 167 \\
\hline
\end{tabular}


Table S9 Indicator: Percentage working in agriculture

\begin{tabular}{|c|c|c|c|c|c|c|c|c|c|c|c|c|c|c|}
\hline \multirow[b]{3}{*}{ Characteristic } & \multirow[b]{3}{*}{ Estimate } & \multirow[b]{3}{*}{ SE } & \multirow{2}{*}{\multicolumn{2}{|c|}{$\begin{array}{r}\text { Male } \\
95 \% \text { C.I }\end{array}$}} & \multirow{3}{*}{$\begin{array}{l}\text { CV } \\
(\%)\end{array}$} & \multirow{3}{*}{$\begin{array}{l}\text { Design } \\
\text { effect }\end{array}$} & \multirow[b]{3}{*}{ Count } & \multirow[b]{3}{*}{ Estimate } & \multirow[b]{3}{*}{ SE } & \multirow{2}{*}{\multicolumn{2}{|c|}{$\begin{array}{l}\text { Female } \\
95 \% \text { C.I }\end{array}$}} & \multirow{3}{*}{$\begin{array}{l}\text { CV } \\
(\%)\end{array}$} & \multirow{3}{*}{$\begin{array}{c}\text { Design } \\
\text { effect }\end{array}$} & \multirow[b]{3}{*}{ Count } \\
\hline & & & & & & & & & & & & & & \\
\hline & & & Lower & Upper & & & & & & Lower & Upper & & & \\
\hline \multicolumn{15}{|l|}{ Urban-rural residence } \\
\hline Urban & 0.035 & 0.009 & 0.017 & 0.053 & 0.259 & 2.074 & 871 & 0.015 & 0.009 & -0.003 & 0.033 & 0.623 & 1.072 & 193 \\
\hline Rural & 0.274 & 0.020 & 0.235 & 0.314 & 0.073 & 3.605 & 1,702 & 0.339 & 0.037 & 0.266 & 0.413 & 0.110 & 1.673 & 310 \\
\hline Informal urban areas & 0.048 & 0.018 & 0.012 & 0.084 & 0.385 & 1.881 & 249 & 0.000 & 0.000 & 0.000 & 0.000 & 0.000 & 0.000 & 47 \\
\hline \multicolumn{15}{|l|}{ Region } \\
\hline Urban Governorates & 0.016 & 0.006 & 0.004 & 0.028 & 0.395 & 1.410 & 566 & 0.003 & 0.003 & -0.003 & 0.009 & 0.990 & 0.423 & 135 \\
\hline Urban Lower Egypt & 0.039 & 0.015 & 0.009 & 0.069 & 0.387 & 1.755 & 287 & 0.000 & 0.000 & 0.000 & 0.000 & 0.000 & 0.000 & 62 \\
\hline Rural Lower Egypt & 0.223 & 0.028 & 0.168 & 0.278 & 0.125 & 4.061 & 907 & 0.208 & 0.043 & 0.124 & 0.293 & 0.206 & 1.421 & 155 \\
\hline Urban Upper Egypt & 0.064 & 0.028 & 0.010 & 0.118 & 0.428 & 2.937 & 149 & 0.092 & 0.065 & -0.036 & 0.220 & 0.707 & 1.264 & 21 \\
\hline Rural Upper Egypt & 0.320 & 0.029 & 0.263 & 0.378 & 0.091 & 3.321 & 636 & 0.457 & 0.055 & 0.349 & 0.565 & 0.121 & 1.707 & 143 \\
\hline Frontier Governorates & 0.390 & 0.081 & 0.231 & 0.549 & 0.207 & 1.559 & 277 & 0.176 & 0.146 & -0.112 & 0.463 & 0.834 & 0.764 & 34 \\
\hline \multicolumn{15}{|l|}{ Age group } \\
\hline $13-17$ years & 0.364 & 0.043 & 0.280 & 0.449 & 0.118 & 1.468 & 200 & 0.653 & 0.108 & 0.442 & 0.865 & 0.165 & 1.312 & 23 \\
\hline $18-24$ years & 0.223 & 0.019 & 0.185 & 0.261 & 0.087 & 2.157 & 957 & 0.188 & 0.040 & 0.110 & 0.266 & 0.211 & 1.641 & 152 \\
\hline $25-29$ years & 0.147 & 0.017 & 0.113 & 0.182 & 0.119 & 2.387 & 933 & 0.142 & 0.030 & 0.083 & 0.200 & 0.210 & 1.132 & 189 \\
\hline $30-35$ years & 0.132 & 0.017 & 0.098 & 0.166 & 0.130 & 1.835 & 732 & 0.168 & 0.032 & 0.106 & 0.231 & 0.190 & 1.082 & 186 \\
\hline \multicolumn{15}{|l|}{ Education level } \\
\hline Illiterate & 0.278 & 0.045 & 0.190 & 0.366 & 0.161 & 2.113 & 223 & 0.676 & 0.075 & 0.527 & 0.824 & 0.112 & 1.381 & 59 \\
\hline Read and write & 0.198 & 0.085 & 0.032 & 0.365 & 0.427 & 1.494 & 35 & 0.292 & 0.204 & -0.108 & 0.692 & 0.697 & 1.177 & 6 \\
\hline Primary & 0.228 & 0.031 & 0.167 & 0.289 & 0.136 & 1.981 & 373 & 0.356 & 0.085 & 0.190 & 0.523 & 0.238 & 1.197 & 47 \\
\hline Preparatory & 0.216 & 0.030 & 0.157 & 0.274 & 0.137 & 1.641 & 310 & 0.282 & 0.086 & 0.113 & 0.452 & 0.306 & 1.645 & 47 \\
\hline General secondary & 0.081 & 0.032 & 0.018 & 0.145 & 0.399 & 1.007 & 63 & 0.225 & 0.139 & -0.049 & 0.498 & 0.621 & 0.837 & 10 \\
\hline Vocational secondary & 0.214 & 0.021 & 0.173 & 0.254 & 0.096 & 3.201 & 1,241 & 0.196 & 0.041 & 0.116 & 0.275 & 0.207 & 1.428 & 153 \\
\hline Post-secondary institute & 0.090 & 0.033 & 0.025 & 0.155 & 0.366 & 1.430 & 97 & 0.068 & 0.047 & -0.025 & 0.160 & 0.694 & 0.816 & 27 \\
\hline University and above & 0.050 & 0.012 & 0.026 & 0.074 & 0.244 & 1.589 & 480 & 0.000 & 0.000 & 0.000 & 0.000 & 0.000 & 0.000 & 201 \\
\hline \multicolumn{15}{|l|}{ Wealth quintile } \\
\hline Lowest & 0.266 & 0.029 & 0.209 & 0.323 & 0.109 & 2.420 & 508 & 0.408 & 0.063 & 0.284 & 0.531 & 0.154 & 1.484 & 91 \\
\hline Second & 0.215 & 0.027 & 0.162 & 0.267 & 0.124 & 2.677 & 581 & 0.210 & 0.044 & 0.124 & 0.296 & 0.208 & 1.133 & 107 \\
\hline Middle & 0.170 & 0.022 & 0.127 & 0.214 & 0.130 & 1.848 & 556 & 0.274 & 0.063 & 0.150 & 0.397 & 0.230 & 1.612 & 95 \\
\hline Fourth & 0.153 & 0.021 & 0.112 & 0.195 & 0.139 & 1.950 & 582 & 0.105 & 0.037 & 0.032 & 0.178 & 0.355 & 1.125 & 92 \\
\hline Highest & 0.114 & 0.016 & 0.082 & 0.145 & 0.142 & 1.549 & 595 & 0.042 & 0.018 & 0.007 & 0.078 & 0.430 & 1.174 & 165 \\
\hline
\end{tabular}


Table S10 Indicator: Percentage working in industry

\begin{tabular}{|c|c|c|c|c|c|c|c|c|c|c|c|c|c|c|}
\hline \multirow[b]{3}{*}{ Characteristic } & \multirow[b]{3}{*}{ Estimate } & \multirow[b]{3}{*}{ SE } & \multicolumn{3}{|c|}{ Male } & \multirow{3}{*}{$\begin{array}{c}\text { Design } \\
\text { effect }\end{array}$} & \multirow[b]{3}{*}{ Count } & \multirow[b]{3}{*}{ Estimate } & \multirow[b]{3}{*}{ SE } & \multirow{2}{*}{\multicolumn{2}{|c|}{ Female }} & \multirow{3}{*}{$\begin{array}{l}\text { CV } \\
\text { (\%) }\end{array}$} & \multirow{3}{*}{$\begin{array}{c}\text { Design } \\
\text { effect }\end{array}$} & \multirow[b]{3}{*}{ Count } \\
\hline & & & 95 & C.I & CV & & & & & & & & & \\
\hline & & & Lower & Upper & (\%) & & & & & Lower & Upper & & & \\
\hline \multicolumn{15}{|l|}{ Urban-rural residence } \\
\hline Urban & 0.691 & 0.020 & 0.650 & 0.731 & 0.030 & 1.670 & 871 & 0.357 & 0.044 & 0.269 & 0.444 & 0.125 & 1.595 & 193 \\
\hline Rural & 0.556 & 0.022 & 0.514 & 0.599 & 0.039 & 3.351 & 1,702 & 0.227 & 0.026 & 0.176 & 0.278 & 0.115 & 1.045 & 310 \\
\hline Informal urban areas & 0.731 & 0.038 & 0.658 & 0.805 & 0.051 & 1.823 & 249 & 0.184 & 0.073 & 0.040 & 0.328 & 0.398 & 1.284 & 47 \\
\hline \multicolumn{15}{|l|}{ Region } \\
\hline Urban Governorates & 0.685 & 0.027 & 0.631 & 0.739 & 0.040 & 1.940 & 566 & 0.405 & 0.051 & 0.304 & 0.506 & 0.127 & 1.503 & 135 \\
\hline Urban Lower Egypt & 0.763 & 0.025 & 0.714 & 0.812 & 0.033 & 0.983 & 287 & 0.275 & 0.077 & 0.124 & 0.426 & 0.280 & 1.655 & 62 \\
\hline Rural Lower Egypt & 0.582 & 0.027 & 0.529 & 0.634 & 0.046 & 2.654 & 907 & 0.288 & 0.037 & 0.216 & 0.361 & 0.128 & 0.842 & 155 \\
\hline Urban Upper Egypt & 0.686 & 0.040 & 0.606 & 0.765 & 0.059 & 1.771 & 149 & 0.051 & 0.051 & -0.048 & 0.151 & 0.990 & 1.314 & 21 \\
\hline Rural Upper Egypt & 0.539 & 0.035 & 0.470 & 0.608 & 0.065 & 4.191 & 636 & 0.174 & 0.037 & 0.101 & 0.248 & 0.214 & 1.350 & 143 \\
\hline Frontier Governorates & 0.362 & 0.062 & 0.241 & 0.482 & 0.170 & 0.929 & 277 & 0.089 & 0.057 & -0.022 & 0.201 & 0.636 & 0.205 & 34 \\
\hline \multicolumn{15}{|l|}{ Age group } \\
\hline 13-17 years & 0.596 & 0.043 & 0.511 & 0.680 & 0.072 & 1.422 & 200 & 0.268 & 0.095 & 0.081 & 0.455 & 0.355 & 1.180 & 23 \\
\hline 18-24 years & 0.613 & 0.022 & 0.570 & 0.655 & 0.035 & 1.989 & 957 & 0.353 & 0.044 & 0.266 & 0.439 & 0.125 & 1.350 & 152 \\
\hline 25-29 years & 0.620 & 0.023 & 0.576 & 0.665 & 0.037 & 2.140 & 933 & 0.230 & 0.039 & 0.154 & 0.306 & 0.169 & 1.323 & 189 \\
\hline $30-35$ years & 0.601 & 0.021 & 0.559 & 0.643 & 0.036 & 1.374 & 732 & 0.233 & 0.038 & 0.158 & 0.309 & 0.164 & 1.216 & 186 \\
\hline \multicolumn{15}{|l|}{ Education level } \\
\hline Illiterate & 0.636 & 0.047 & 0.544 & 0.729 & 0.074 & 2.043 & 223 & 0.262 & 0.070 & 0.123 & 0.400 & 0.269 & 1.363 & 59 \\
\hline Read and write & 0.638 & 0.095 & 0.452 & 0.825 & 0.149 & 1.294 & 35 & 0.261 & 0.176 & -0.085 & 0.607 & 0.673 & 0.943 & 6 \\
\hline Primary & 0.688 & 0.033 & 0.623 & 0.754 & 0.049 & 1.884 & 373 & 0.426 & 0.080 & 0.269 & 0.583 & 0.188 & 0.998 & 47 \\
\hline Preparatory & 0.646 & 0.034 & 0.580 & 0.712 & 0.052 & 1.557 & 310 & 0.549 & 0.081 & 0.390 & 0.707 & 0.147 & 1.172 & 47 \\
\hline General secondary & 0.738 & 0.052 & 0.636 & 0.841 & 0.071 & 1.010 & 63 & 0.457 & 0.164 & 0.135 & 0.779 & 0.358 & 0.812 & 10 \\
\hline Vocational secondary & 0.613 & 0.023 & 0.568 & 0.657 & 0.037 & 2.781 & 1,241 & 0.374 & 0.046 & 0.283 & 0.465 & 0.124 & 1.251 & 153 \\
\hline Post-secondary institute & 0.599 & 0.060 & 0.482 & 0.717 & 0.100 & 1.605 & 97 & 0.080 & 0.046 & -0.011 & 0.170 & 0.578 & 0.674 & 27 \\
\hline University and above & 0.504 & 0.028 & 0.449 & 0.559 & 0.056 & 1.593 & 480 & 0.117 & 0.028 & 0.062 & 0.173 & 0.242 & 1.395 & 201 \\
\hline \multicolumn{15}{|l|}{ Wealth quintile } \\
\hline Lowest & 0.606 & 0.034 & 0.539 & 0.672 & 0.056 & 2.683 & 508 & 0.412 & 0.064 & 0.285 & 0.538 & 0.156 & 1.552 & 91 \\
\hline Second & 0.620 & 0.029 & 0.562 & 0.678 & 0.047 & 2.317 & 581 & 0.387 & 0.052 & 0.284 & 0.490 & 0.135 & 1.136 & 107 \\
\hline Middle & 0.618 & 0.027 & 0.565 & 0.672 & 0.044 & 1.704 & 556 & 0.285 & 0.055 & 0.177 & 0.393 & 0.192 & 1.194 & 95 \\
\hline Fourth & 0.627 & 0.026 & 0.577 & 0.678 & 0.041 & 1.578 & 582 & 0.242 & 0.051 & 0.142 & 0.343 & 0.211 & 1.080 & 92 \\
\hline Highest & 0.586 & 0.026 & 0.535 & 0.638 & 0.045 & 1.691 & 595 & 0.116 & 0.027 & 0.063 & 0.169 & 0.232 & 1.014 & 165 \\
\hline
\end{tabular}


Table S11 Indicator: Percentage working in services

\begin{tabular}{|c|c|c|c|c|c|c|c|c|c|c|c|c|c|c|}
\hline \multirow[b]{3}{*}{ Characteristic } & \multirow[b]{3}{*}{ Estimate } & \multirow[b]{3}{*}{ SE } & \multicolumn{3}{|c|}{ Male } & \multirow{3}{*}{$\begin{array}{c}\text { Design } \\
\text { effect }\end{array}$} & \multirow[b]{3}{*}{ Count } & \multirow[b]{3}{*}{ Estimate } & \multirow[b]{3}{*}{ SE } & \multirow{2}{*}{\multicolumn{2}{|c|}{$\begin{array}{l}\text { Female } \\
95 \% \text { C.I }\end{array}$}} & \multirow{3}{*}{$\begin{array}{l}\text { CV } \\
\text { (\%) }\end{array}$} & \multirow{3}{*}{$\begin{array}{l}\text { Design } \\
\text { effect }\end{array}$} & \multirow[b]{3}{*}{ Count } \\
\hline & & & 95 & C.I & CV & & & & & & & & & \\
\hline & & & Lower & Upper & (\%) & & & & & Lower & Upper & & & \\
\hline \multicolumn{15}{|l|}{ Urban-rural residence } \\
\hline Urban & 0.274 & 0.020 & 0.236 & 0.313 & 0.072 & 1.668 & 871 & 0.629 & 0.045 & 0.540 & 0.717 & 0.072 & 1.620 & 193 \\
\hline Rural & 0.170 & 0.013 & 0.144 & 0.195 & 0.076 & 2.101 & 1,702 & 0.434 & 0.036 & 0.363 & 0.504 & 0.083 & 1.417 & 310 \\
\hline Informal urban areas & 0.221 & 0.038 & 0.146 & 0.296 & 0.174 & 2.173 & 249 & 0.816 & 0.073 & 0.672 & 0.960 & 0.090 & 1.284 & 47 \\
\hline \multicolumn{15}{|l|}{ Region } \\
\hline Urban Governorates & 0.299 & 0.027 & 0.245 & 0.353 & 0.092 & 1.986 & 566 & 0.592 & 0.052 & 0.489 & 0.694 & 0.088 & 1.537 & 135 \\
\hline Urban Lower Egypt & 0.198 & 0.025 & 0.148 & 0.248 & 0.128 & 1.159 & 287 & 0.725 & 0.077 & 0.574 & 0.876 & 0.106 & 1.655 & 62 \\
\hline Rural Lower Egypt & 0.195 & 0.018 & 0.160 & 0.231 & 0.092 & 1.837 & 907 & 0.503 & 0.042 & 0.420 & 0.587 & 0.084 & 0.909 & 155 \\
\hline Urban Upper Egypt & 0.250 & 0.037 & 0.177 & 0.322 & 0.148 & 1.700 & 149 & 0.857 & 0.082 & 0.697 & 1.017 & 0.095 & 1.347 & 21 \\
\hline Rural Upper Egypt & 0.141 & 0.019 & 0.105 & 0.178 & 0.132 & 2.408 & 636 & 0.369 & 0.055 & 0.261 & 0.476 & 0.148 & 1.792 & 143 \\
\hline Frontier Governorates & 0.249 & 0.035 & 0.179 & 0.318 & 0.142 & 0.380 & 277 & 0.735 & 0.135 & 0.470 & 1.001 & 0.184 & 0.484 & 34 \\
\hline \multicolumn{15}{|l|}{ Age group } \\
\hline $13-17$ years & 0.040 & 0.014 & 0.012 & 0.068 & 0.361 & 1.000 & 200 & 0.079 & 0.058 & -0.034 & 0.192 & 0.731 & 1.169 & 23 \\
\hline $18-24$ years & 0.165 & 0.015 & 0.136 & 0.194 & 0.089 & 1.566 & 957 & 0.460 & 0.048 & 0.365 & 0.554 & 0.105 & 1.485 & 152 \\
\hline $25-29$ years & 0.233 & 0.017 & 0.200 & 0.265 & 0.071 & 1.501 & 933 & 0.628 & 0.042 & 0.545 & 0.712 & 0.068 & 1.204 & 189 \\
\hline $30-35$ years & 0.267 & 0.019 & 0.229 & 0.304 & 0.071 & 1.316 & 732 & 0.598 & 0.045 & 0.510 & 0.686 & 0.075 & 1.241 & 186 \\
\hline \multicolumn{15}{|l|}{ Education level } \\
\hline Illiterate & 0.085 & 0.022 & 0.043 & 0.128 & 0.253 & 1.275 & 223 & 0.062 & 0.038 & -0.013 & 0.138 & 0.616 & 1.338 & 59 \\
\hline Read and write & 0.163 & 0.065 & 0.035 & 0.291 & 0.399 & 1.028 & 35 & 0.446 & 0.236 & -0.017 & 0.910 & 0.528 & 1.322 & 6 \\
\hline Primary & 0.084 & 0.018 & 0.048 & 0.120 & 0.218 & 1.567 & 373 & 0.218 & 0.060 & 0.099 & 0.337 & 0.277 & 0.818 & 47 \\
\hline Preparatory & 0.139 & 0.024 & 0.091 & 0.187 & 0.175 & 1.563 & 310 & 0.169 & 0.059 & 0.054 & 0.284 & 0.347 & 1.089 & 47 \\
\hline General secondary & 0.180 & 0.045 & 0.092 & 0.269 & 0.250 & 0.980 & 63 & 0.318 & 0.144 & 0.036 & 0.601 & 0.452 & 0.716 & 10 \\
\hline Vocational secondary & 0.174 & 0.015 & 0.145 & 0.202 & 0.084 & 1.879 & 1,241 & 0.431 & 0.046 & 0.341 & 0.520 & 0.106 & 1.157 & 153 \\
\hline Post-secondary institute & 0.310 & 0.055 & 0.202 & 0.418 & 0.177 & 1.518 & 97 & 0.853 & 0.065 & 0.726 & 0.980 & 0.076 & 0.774 & 27 \\
\hline University and above & 0.446 & 0.027 & 0.393 & 0.500 & 0.061 & 1.501 & 480 & 0.883 & 0.028 & 0.827 & 0.938 & 0.032 & 1.395 & 201 \\
\hline \multicolumn{15}{|l|}{ Wealth quintile } \\
\hline Lowest & 0.128 & 0.017 & 0.095 & 0.162 & 0.132 & 1.433 & 508 & 0.181 & 0.050 & 0.083 & 0.278 & 0.275 & 1.515 & 91 \\
\hline Second & 0.166 & 0.019 & 0.129 & 0.202 & 0.113 & 1.610 & 581 & 0.403 & 0.055 & 0.294 & 0.512 & 0.137 & 1.255 & 107 \\
\hline Middle & 0.211 & 0.023 & 0.165 & 0.257 & 0.111 & 1.756 & 556 & 0.441 & 0.063 & 0.317 & 0.566 & 0.144 & 1.321 & 95 \\
\hline Fourth & 0.219 & 0.021 & 0.178 & 0.261 & 0.097 & 1.472 & 582 & 0.653 & 0.056 & 0.544 & 0.762 & 0.085 & 1.035 & 92 \\
\hline Highest & 0.300 & 0.025 & 0.252 & 0.348 & 0.082 & 1.718 & 595 & 0.842 & 0.031 & 0.781 & 0.902 & 0.036 & 1.009 & 165 \\
\hline
\end{tabular}


Table S12 Indicator: Percentage working in private sector

\begin{tabular}{|c|c|c|c|c|c|c|c|c|c|c|c|c|c|c|}
\hline \multirow[b]{3}{*}{ Characteristic } & \multirow[b]{3}{*}{ Estimate } & \multirow[b]{3}{*}{ SE } & \multicolumn{3}{|c|}{ Male } & \multirow{3}{*}{$\begin{array}{c}\text { Design } \\
\text { effect }\end{array}$} & \multirow[b]{3}{*}{ Count } & \multirow[b]{3}{*}{ Estimate } & \multirow[b]{3}{*}{ SE } & \multicolumn{2}{|c|}{ Female } & \multirow{3}{*}{$\begin{array}{l}\text { CV } \\
(\%)\end{array}$} & \multirow{3}{*}{$\begin{array}{l}\text { Design } \\
\text { effect }\end{array}$} & \multirow[b]{3}{*}{ Count } \\
\hline & & & \multicolumn{2}{|c|}{ 95\% C.I } & \multirow{2}{*}{$\begin{array}{l}\text { CV } \\
\text { (\%) }\end{array}$} & & & & & \multicolumn{2}{|c|}{ 95\% C.I } & & & \\
\hline & & & Lower & Upper & & & & & & Lower & Upper & & & \\
\hline \multicolumn{15}{|l|}{ Urban-rural residence } \\
\hline Urban & 0.846 & 0.016 & 0.815 & 0.878 & 0.019 & 1.659 & 873 & 0.657 & 0.044 & 0.572 & 0.743 & 0.066 & 1.573 & 194 \\
\hline Rural & 0.874 & 0.009 & 0.855 & 0.892 & 0.011 & 1.401 & 1,706 & 0.680 & 0.031 & 0.618 & 0.741 & 0.046 & 1.218 & 310 \\
\hline Informal urban areas & 0.893 & 0.022 & 0.849 & 0.936 & 0.025 & 1.305 & 250 & 0.384 & 0.104 & 0.179 & 0.589 & 0.272 & 1.661 & 47 \\
\hline \multicolumn{15}{|l|}{ Region } \\
\hline Urban Governorates & 0.851 & 0.020 & 0.812 & 0.891 & 0.023 & 1.760 & 568 & 0.761 & 0.041 & 0.680 & 0.842 & 0.054 & 1.287 & 136 \\
\hline Urban Lower Egypt & 0.889 & 0.022 & 0.846 & 0.932 & 0.024 & 1.376 & 288 & 0.420 & 0.087 & 0.250 & 0.590 & 0.206 & 1.712 & 62 \\
\hline Rural Lower Egypt & 0.856 & 0.014 & 0.828 & 0.883 & 0.016 & 1.444 & 910 & 0.604 & 0.042 & 0.522 & 0.687 & 0.070 & 0.939 & 155 \\
\hline Urban Upper Egypt & 0.837 & 0.032 & 0.774 & 0.901 & 0.039 & 1.793 & 149 & 0.289 & 0.100 & 0.093 & 0.485 & 0.345 & 1.203 & 21 \\
\hline Rural Upper Egypt & 0.893 & 0.012 & 0.869 & 0.917 & 0.014 & 1.342 & 637 & 0.750 & 0.042 & 0.668 & 0.831 & 0.055 & 1.281 & 143 \\
\hline Frontier Governorates & 0.841 & 0.031 & 0.779 & 0.902 & 0.037 & 0.412 & 277 & 0.284 & 0.155 & -0.021 & 0.590 & 0.547 & 0.613 & 34 \\
\hline \multicolumn{15}{|l|}{ Age group } \\
\hline $13-17$ years & 0.987 & 0.008 & 0.972 & 1.002 & 0.008 & 0.831 & 201 & 0.000 & 0.000 & 0.000 & 0.000 & 0.000 & 0.000 & 23 \\
\hline $18-24$ years & 0.932 & 0.009 & 0.915 & 0.950 & 0.010 & 1.253 & 961 & 0.780 & 0.039 & 0.702 & 0.857 & 0.051 & 1.447 & 153 \\
\hline $25-29$ years & 0.828 & 0.014 & 0.801 & 0.855 & 0.017 & 1.302 & 934 & 0.588 & 0.042 & 0.506 & 0.671 & 0.072 & 1.143 & 189 \\
\hline $30-35$ years & 0.798 & 0.018 & 0.764 & 0.833 & 0.022 & 1.370 & 733 & 0.513 & 0.044 & 0.426 & 0.599 & 0.086 & 1.154 & 186 \\
\hline \multicolumn{15}{|l|}{ Education level } \\
\hline Illiterate & 0.953 & 0.017 & 0.920 & 0.986 & 0.018 & 1.316 & 223 & 0.986 & 0.014 & 0.958 & 1.014 & 0.014 & 0.767 & 59 \\
\hline Read and write & 0.922 & 0.046 & 0.832 & 1.011 & 0.049 & 0.948 & 35 & 0.884 & 0.115 & 0.657 & 1.110 & 0.131 & 0.759 & 6 \\
\hline Primary & 0.948 & 0.014 & 0.921 & 0.975 & 0.015 & 1.411 & 373 & 0.919 & 0.039 & 0.842 & 0.995 & 0.043 & 0.778 & 47 \\
\hline Preparatory & 0.948 & 0.015 & 0.919 & 0.976 & 0.015 & 1.358 & 311 & 0.960 & 0.028 & 0.905 & 1.014 & 0.029 & 0.889 & 47 \\
\hline General secondary & 0.904 & 0.035 & 0.835 & 0.973 & 0.039 & 1.021 & 63 & 0.946 & 0.054 & 0.839 & 1.052 & 0.057 & 0.430 & 10 \\
\hline Vocational secondary & 0.884 & 0.010 & 0.864 & 0.903 & 0.011 & 1.233 & 1,246 & 0.689 & 0.044 & 0.602 & 0.776 & 0.064 & 1.249 & 153 \\
\hline Post-secondary institute & 0.789 & 0.043 & 0.704 & 0.874 & 0.055 & 1.205 & 97 & 0.432 & 0.111 & 0.214 & 0.649 & 0.256 & 1.161 & 27 \\
\hline University and above & 0.690 & 0.024 & 0.643 & 0.737 & 0.035 & 1.355 & 481 & 0.396 & 0.046 & 0.306 & 0.486 & 0.116 & 1.587 & 202 \\
\hline \multicolumn{15}{|l|}{ Wealth quintile } \\
\hline Lowest & 0.918 & 0.014 & 0.889 & 0.946 & 0.016 & 1.517 & 509 & 0.855 & 0.049 & 0.759 & 0.951 & 0.057 & 1.743 & 91 \\
\hline Second & 0.899 & 0.014 & 0.871 & 0.926 & 0.015 & 1.330 & 582 & 0.787 & 0.041 & 0.706 & 0.869 & 0.053 & 1.010 & 107 \\
\hline Middle & 0.887 & 0.017 & 0.855 & 0.920 & 0.019 & 1.474 & 557 & 0.702 & 0.053 & 0.598 & 0.806 & 0.076 & 1.087 & 95 \\
\hline Fourth & 0.842 & 0.018 & 0.807 & 0.877 & 0.021 & 1.357 & 584 & 0.654 & 0.053 & 0.549 & 0.759 & 0.082 & 0.959 & 92 \\
\hline Highest & 0.793 & 0.020 & 0.754 & 0.831 & 0.025 & 1.393 & 597 & 0.395 & 0.047 & 0.302 & 0.487 & 0.119 & 1.335 & 166 \\
\hline
\end{tabular}


Table S13 Indicator: Percentage of employers and self-employed workers

\begin{tabular}{|c|c|c|c|c|c|c|c|c|c|c|c|c|c|c|}
\hline \multirow[b]{3}{*}{ Characteristic } & \multirow[b]{3}{*}{ Estimate } & \multirow[b]{3}{*}{ SE } & \multirow{2}{*}{\multicolumn{2}{|c|}{$\begin{aligned} \text { Male } \\
95 \% \text { C.I }\end{aligned}$}} & \multirow{3}{*}{$\begin{array}{l}\text { CV } \\
(\%)\end{array}$} & \multirow{3}{*}{$\begin{array}{l}\text { Design } \\
\text { effect }\end{array}$} & \multirow[b]{3}{*}{ Count } & \multirow[b]{3}{*}{ Estimate } & \multirow[b]{3}{*}{ SE } & \multirow{2}{*}{\multicolumn{2}{|c|}{$\begin{array}{l}\text { Female } \\
95 \% \text { C.I }\end{array}$}} & \multirow{3}{*}{$\begin{array}{l}\text { CV } \\
(\%)\end{array}$} & \multirow{3}{*}{$\begin{array}{l}\text { Design } \\
\text { effect }\end{array}$} & \multirow[b]{3}{*}{ Count } \\
\hline & & & & & & & & & & & & & & \\
\hline & & & Lower & Upper & & & & & & Lower & Upper & & & \\
\hline \multicolumn{15}{|l|}{ Urban-rural residence } \\
\hline Urban & 0.148 & 0.015 & 0.119 & 0.176 & 0.098 & 1.425 & 871 & 0.051 & 0.018 & 0.150 & 0.086 & 0.358 & 1.282 & 195 \\
\hline Rural & 0.146 & 0.015 & 0.126 & 0.167 & 0.105 & 1.585 & 1,706 & 0.120 & 0.018 & 0.083 & 0.156 & 0.155 & 0.871 & 310 \\
\hline Informal urban areas & 0.260 & 0.039 & 0.183 & 0.337 & 0.151 & 2.031 & 250 & 0.160 & 0.070 & 0.225 & 0.298 & 0.437 & 1.314 & 47 \\
\hline \multicolumn{15}{|l|}{ Region } \\
\hline Urban Governorates & 0.146 & 0.018 & 0.111 & 0.181 & 0.122 & 1.417 & 567 & 0.058 & 0.025 & 0.082 & 0.108 & 0.437 & 1.655 & 137 \\
\hline Urban Lower Egypt & 0.257 & 0.034 & 0.189 & 0.324 & 0.134 & 1.779 & 288 & 0.084 & 0.037 & 0.113 & 0.156 & 0.440 & 0.983 & 62 \\
\hline Rural Lower Egypt & 0.180 & 0.015 & 0.151 & 0.210 & 0.082 & 1.351 & 910 & 0.092 & 0.024 & 0.044 & 0.140 & 0.266 & 0.909 & 155 \\
\hline Urban Upper Egypt & 0.140 & 0.032 & 0.780 & 0.203 & 0.226 & 1.947 & 149 & 0.083 & 0.058 & 0.031 & 0.196 & 0.698 & 1.092 & 21 \\
\hline Rural Upper Egypt & 0.108 & 0.014 & 0.081 & 0.135 & 0.129 & 1.693 & 637 & 0.146 & 0.027 & 0.094 & 0.198 & 0.182 & 0.791 & 143 \\
\hline Frontier Governorates & 0.172 & 0.031 & 0.111 & 0.233 & 0.180 & 0.382 & 276 & 0.082 & 0.055 & 0.026 & 0.189 & 0.671 & 0.206 & 34 \\
\hline \multicolumn{15}{|l|}{ Age group } \\
\hline $13-17$ years & 0.043 & 0.015 & 0.015 & 0.134 & 0.348 & 0.998 & 201 & 0.055 & 0.054 & -0.014 & 0.051 & 0.977 & 1.428 & 23 \\
\hline $18-24$ years & 0.120 & 0.013 & 0.013 & 0.195 & 0.109 & 1.637 & 961 & 0.051 & 0.020 & 0.020 & 0.126 & 0.384 & 1.272 & 153 \\
\hline $25-29$ years & 0.165 & 0.016 & 0.016 & 0.134 & 0.096 & 1.771 & 933 & 0.081 & 0.021 & 0.012 & 0.383 & 0.267 & 0.985 & 190 \\
\hline $30-35$ years & 0.227 & 0.019 & 0.019 & 0.190 & 0.083 & 1.439 & 732 & 0.168 & 0.027 & 0.017 & 0.115 & 0.161 & 0.778 & 186 \\
\hline \multicolumn{15}{|l|}{ Education level } \\
\hline Illiterate & 0.159 & 0.031 & 0.097 & 0.221 & 0.198 & 1.574 & 223 & 0.222 & 0.059 & 0.106 & 0.338 & 0.265 & 1.062 & 59 \\
\hline Read and write & 0.213 & 0.072 & 0.070 & 0.355 & 0.341 & 1.038 & 35 & 0.150 & 0.145 & 0.136 & 0.436 & 0.970 & 0.910 & 5 \\
\hline Primary & 0.200 & 0.024 & 0.153 & 0.247 & 0.120 & 1.295 & 372 & 0.114 & 0.050 & 0.016 & 0.212 & 0.436 & 0.934 & 47 \\
\hline Preparatory & 0.159 & 0.025 & 0.110 & 0.208 & 0.156 & 1.476 & 310 & 0.085 & 0.042 & 0.026 & 0.168 & 0.493 & 1.021 & 48 \\
\hline General secondary & 0.166 & 0.055 & 0.059 & 0.274 & 0.329 & 1.538 & 63 & 0.000 & 0.000 & 0.000 & 0.000 & 0.000 & 0.000 & 10 \\
\hline Vocational secondary & 0.142 & 0.012 & 0.119 & 0.166 & 0.084 & 1.502 & 1,246 & 0.100 & 0.025 & 0.050 & 0.150 & 0.255 & 0.988 & 153 \\
\hline Post-secondary institute & 0.099 & 0.034 & 0.033 & 0.165 & 0.340 & 1.359 & 97 & 0.129 & 0.063 & 0.053 & 0.252 & 0.488 & 0.818 & 27 \\
\hline University and above & 0.168 & 0.022 & 0.125 & 0.210 & 0.129 & 1.709 & 481 & 0.054 & 0.018 & 0.019 & 0.089 & 0.329 & 1.131 & 203 \\
\hline \multicolumn{15}{|l|}{ Wealth quintile } \\
\hline Lowest & 0.100 & 0.014 & 0.072 & 0.127 & 0.139 & 1.204 & 509 & 0.177 & 0.040 & 0.098 & 0.256 & 0.227 & 1.005 & 91 \\
\hline Second & 0.146 & 0.017 & 0.113 & 0.179 & 0.115 & 1.441 & 581 & 0.076 & 0.028 & 0.021 & 0.132 & 0.371 & 1.121 & 107 \\
\hline Middle & 0.200 & 0.021 & 0.159 & 0.240 & 0.103 & 1.409 & 556 & 0.102 & 0.029 & 0.045 & 0.160 & 0.286 & 0.755 & 96 \\
\hline Fourth & 0.149 & 0.018 & 0.113 & 0.184 & 0.121 & 1.435 & 584 & 0.093 & 0.030 & 0.034 & 0.152 & 0.322 & 0.806 & 91 \\
\hline Highest & 0.191 & 0.019 & 0.153 & 0.228 & 0.100 & 1.406 & 597 & 0.057 & 0.018 & 0.023 & 0.092 & 0.309 & 0.850 & 167 \\
\hline
\end{tabular}


Table S14 Indicator: Percentage of unpaid family workers

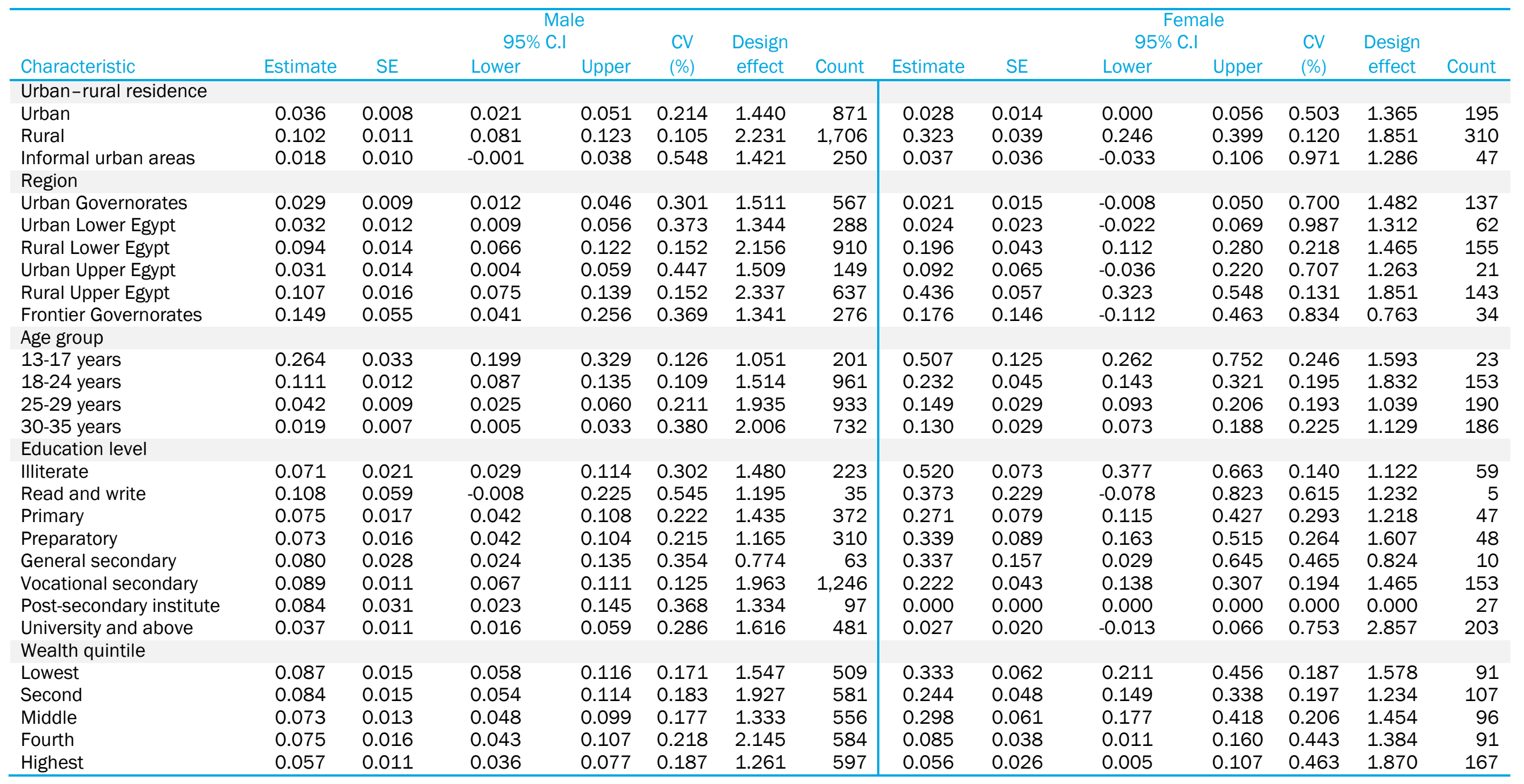


Table S15 Indicator: Proportion of youth dropped out of school

\begin{tabular}{|c|c|c|c|c|c|c|c|c|c|c|c|c|c|c|}
\hline \multirow[b]{2}{*}{ Characteristic } & \multirow[b]{2}{*}{ Estimate } & \multirow[b]{2}{*}{ SE } & \multicolumn{2}{|c|}{$\begin{aligned} \text { Male } \\
95 \% \text { C.I }\end{aligned}$} & \multirow{2}{*}{$\begin{array}{l}\text { CV } \\
\text { (\%) }\end{array}$} & \multirow{2}{*}{$\begin{array}{c}\text { Design } \\
\text { effect }\end{array}$} & \multirow[b]{2}{*}{ Count } & \multirow[b]{2}{*}{ Estimate } & \multirow[b]{2}{*}{ SE } & \multicolumn{2}{|c|}{$\begin{array}{l}\text { Female } \\
95 \% \text { C.I }\end{array}$} & \multirow{2}{*}{$\begin{array}{l}\text { CV } \\
(\%)\end{array}$} & \multirow{2}{*}{$\begin{array}{c}\text { Design } \\
\text { effect }\end{array}$} & \multirow[b]{2}{*}{ Count } \\
\hline & & & Lower & Upper & & & & & & Lower & Upper & & & \\
\hline \multicolumn{15}{|l|}{ Urban-rural residence } \\
\hline Urban & 0.192 & 0.017 & 0.158 & 0.226 & 0.090 & 3.227 & 1,622 & 0.182 & 0.015 & 0.152 & 0.212 & 0.084 & 2.391 & 1,692 \\
\hline Rural & 0.178 & 0.010 & 0.158 & 0.197 & 0.055 & 2.205 & 2,957 & 0.211 & 0.011 & 0.189 & 0.232 & 0.052 & 2.360 & 3,546 \\
\hline Informal urban areas & 0.148 & 0.029 & 0.090 & 0.206 & 0.199 & 3.665 & 494 & 0.130 & 0.022 & 0.086 & 0.174 & 0.171 & 2.360 & 605 \\
\hline \multicolumn{15}{|l|}{ Region } \\
\hline Urban Governorates & 0.231 & 0.023 & 0.187 & 0.276 & 0.098 & 3.134 & 1,025 & 0.217 & 0.019 & 0.180 & 0.253 & 0.086 & 1.964 & 1,039 \\
\hline Urban Lower Egypt & 0.131 & 0.022 & 0.087 & 0.174 & 0.170 & 2.760 & 576 & 0.114 & 0.018 & 0.079 & 0.150 & 0.159 & 2.053 & 641 \\
\hline Rural Lower Egypt & 0.192 & 0.012 & 0.169 & 0.216 & 0.063 & 1.671 & 1,618 & 0.167 & 0.014 & 0.140 & 0.194 & 0.081 & 2.154 & 1,808 \\
\hline Urban Upper Egypt & 0.142 & 0.030 & 0.082 & 0.202 & 0.214 & 3.441 & 289 & 0.142 & 0.035 & 0.074 & 0.209 & 0.244 & 4.171 & 350 \\
\hline Rural Upper Egypt & 0.161 & 0.016 & 0.130 & 0.193 & 0.099 & 2.913 & 1,110 & 0.255 & 0.017 & 0.222 & 0.288 & 0.065 & 2.331 & 1,509 \\
\hline Frontier Governorates & 0.134 & 0.026 & 0.082 & 0.185 & 0.195 & 0.600 & 455 & 0.183 & 0.043 & 0.099 & 0.267 & 0.234 & 1.177 & 496 \\
\hline \multicolumn{15}{|l|}{ Age group } \\
\hline $13-17$ years & 0.113 & 0.011 & 0.091 & 0.136 & 0.099 & 1.393 & 1,091 & 0.165 & 0.015 & 0.135 & 0.195 & 0.093 & 1.925 & 1,105 \\
\hline $18-24$ years & 0.195 & 0.012 & 0.172 & 0.217 & 0.059 & 1.970 & 2,080 & 0.200 & 0.011 & 0.178 & 0.222 & 0.056 & 1.575 & 2,078 \\
\hline $25-29$ years & 0.175 & 0.014 & 0.147 & 0.204 & 0.082 & 1.855 & 1,108 & 0.203 & 0.013 & 0.176 & 0.228 & 0.066 & 1.376 & 1,456 \\
\hline $30-35$ years & 0.227 & 0.019 & 0.190 & 0.264 & 0.083 & 1.740 & 794 & 0.206 & 0.014 & 0.179 & 0.233 & 0.067 & 1.139 & 1,204 \\
\hline \multicolumn{15}{|l|}{ Education level } \\
\hline Illiterate & 0.000 & 0.000 & 0.000 & 0.000 & 0.000 & 0.000 & 288 & 0.000 & 0.000 & 0.000 & 0.000 & 0.000 & 0.000 & 831 \\
\hline Read and write & 0.000 & 0.000 & 0.000 & 0.000 & 0.000 & 0.000 & 38 & 0.000 & 0.000 & 0.000 & 0.000 & 0.000 & 0.000 & 83 \\
\hline Primary & 0.958 & 0.011 & 0.935 & 0.098 & 0.012 & 1.582 & 455 & 0.983 & 0.006 & 0.971 & 0.995 & 0.006 & 0.866 & 439 \\
\hline Preparatory & 0.531 & 0.025 & 0.483 & 0.580 & 0.047 & 1.725 & 646 & 0.596 & 0.022 & 0.552 & 0.639 & 0.037 & 1.653 & 872 \\
\hline General secondary & 0.115 & 0.022 & 0.073 & 0.158 & 0.188 & 2.022 & 417 & 0.101 & 0.014 & 0.073 & 0.129 & 0.141 & 1.076 & 516 \\
\hline Vocational secondary & 0.038 & 0.005 & 0.028 & 0.048 & 0.137 & 1.681 & 2,027 & 0.058 & 0.007 & 0.045 & 0.072 & 0.116 & 1.466 & 1,952 \\
\hline Post-secondary institute & 0.024 & 0.011 & 0.002 & 0.045 & 0.456 & 1.028 & 188 & 0.030 & 0.014 & 0.001 & 0.050 & 0.485 & 1.099 & 165 \\
\hline University and above & 0.014 & 0.005 & 0.004 & 0.023 & 0.350 & 1.950 & 1,014 & 0.016 & 0.004 & 0.008 & 0.024 & 0.258 & 1.088 & 982 \\
\hline \multicolumn{15}{|l|}{ Wealth quintile } \\
\hline Lowest & 0.253 & 0.021 & 0.211 & 0.295 & 0.085 & 2.393 & 829 & 0.271 & 0.019 & 0.234 & 0.309 & 0.070 & 1.830 & 1,021 \\
\hline Second & 0.242 & 0.018 & 0.206 & 0.279 & 0.076 & 2.081 & 954 & 0.244 & 0.016 & 0.212 & 0.276 & 0.067 & 1.565 & 1,145 \\
\hline Middle & 0.202 & 0.015 & 0.171 & 0.232 & 0.076 & 1.509 & 987 & 0.228 & 0.015 & 0.199 & 0.257 & 0.065 & 1.256 & 1,165 \\
\hline Fourth & 0.137 & 0.013 & 0.111 & 0.162 & 0.095 & 1.627 & 1,082 & 0.168 & 0.014 & 0.142 & 0.195 & 0.080 & 1.341 & 1,192 \\
\hline Highest & 0.089 & 0.011 & 0.068 & 0.110 & 0.118 & 1.811 & 1,221 & 0.080 & 0.008 & 0.064 & 0.096 & 0.101 & 1.086 & 1,320 \\
\hline
\end{tabular}


Table S16 Indicator: Proportion aspiring to migrate

\begin{tabular}{|c|c|c|c|c|c|c|c|c|c|c|c|c|c|c|}
\hline \multirow[b]{3}{*}{ Characteristic } & \multirow[b]{3}{*}{ Estimate } & \multirow[b]{3}{*}{ SE } & \multicolumn{3}{|c|}{ Male } & \multirow{3}{*}{$\begin{array}{c}\text { Design } \\
\text { effect }\end{array}$} & \multirow[b]{3}{*}{ Count } & \multirow[b]{3}{*}{ Estimate } & \multirow[b]{3}{*}{ SE } & \multirow{2}{*}{\multicolumn{2}{|c|}{$\begin{array}{l}\text { Female } \\
95 \% \text { C.I }\end{array}$}} & \multirow{3}{*}{$\begin{array}{l}\text { CV } \\
\text { (\%) }\end{array}$} & \multirow{3}{*}{$\begin{array}{c}\text { Design } \\
\text { effect }\end{array}$} & \multirow[b]{3}{*}{ Count } \\
\hline & & & 95 & C.I & CV & & & & & & & & & \\
\hline & & & Lower & Upper & $(\%)$ & & & & & Lower & Upper & & & \\
\hline \multicolumn{15}{|l|}{ Urban-rural residence } \\
\hline Urban & 0.255 & 0.019 & 0.218 & 0.292 & 0.073 & 3.032 & 1,573 & 0.094 & 0.010 & 0.075 & 0.114 & 0.107 & 1.779 & 1,643 \\
\hline Rural & 0.286 & 0.014 & 0.259 & 0.313 & 0.048 & 3.073 & 2,880 & 0.078 & 0.009 & 0.061 & 0.095 & 0.110 & 3.224 & 3,436 \\
\hline Informal urban areas & 0.145 & 0.027 & 0.091 & 0.198 & 0.188 & 3.158 & 488 & 0.037 & 0.009 & 0.019 & 0.056 & 0.255 & 1.336 & 601 \\
\hline \multicolumn{15}{|l|}{ Region } \\
\hline Urban Governorates & 0.248 & 0.019 & 0.210 & 0.285 & 0.077 & 2.030 & 995 & 0.115 & 0.013 & 0.089 & 0.140 & 0.112 & 1.537 & 1,007 \\
\hline Urban Lower Egypt & 0.226 & 0.032 & 0.162 & 0.289 & 0.142 & 3.660 & 561 & 0.053 & 0.011 & 0.031 & 0.076 & 0.214 & 1.589 & 634 \\
\hline Rural Lower Egypt & 0.295 & 0.018 & 0.260 & 0.331 & 0.062 & 2.787 & 1,580 & 0.055 & 0.008 & 0.040 & 0.070 & 0.138 & 1.721 & 1,742 \\
\hline Urban Upper Egypt & 0.193 & 0.046 & 0.102 & 0.285 & 0.241 & 6.223 & 285 & 0.040 & 0.016 & 0.010 & 0.071 & 0.387 & 2.616 & 343 \\
\hline Rural Upper Egypt & 0.278 & 0.022 & 0.235 & 0.320 & 0.078 & 3.462 & 1,076 & 0.102 & 0.015 & 0.072 & 0.132 & 0.150 & 3.955 & 1,468 \\
\hline Frontier Governorates & 0.197 & 0.028 & 0.142 & 0.251 & 0.141 & 0.486 & 444 & 0.054 & 0.015 & 0.024 & 0.083 & 0.279 & 0.416 & 486 \\
\hline \multicolumn{15}{|l|}{ Age group } \\
\hline $13-17$ years & 0.209 & 0.016 & 0.178 & 0.240 & 0.076 & 1.466 & 959 & 0.088 & 0.012 & 0.064 & 0.112 & 0.138 & 1.761 & 942 \\
\hline 18-24 years & 0.299 & 0.014 & 0.270 & 0.327 & 0.048 & 2.275 & 2,080 & 0.093 & 0.009 & 0.075 & 0.111 & 0.100 & 2.059 & 2,078 \\
\hline $25-29$ years & 0.273 & 0.017 & 0.239 & 0.307 & 0.063 & 1.957 & 1,108 & 0.064 & 0.008 & 0.048 & 0.079 & 0.124 & 1.299 & 1,456 \\
\hline $30-35$ years & 0.214 & 0.017 & 0.180 & 0.249 & 0.081 & 1.572 & 794 & 0.058 & 0.008 & 0.043 & 0.074 & 0.138 & 1.157 & 1,204 \\
\hline \multicolumn{15}{|l|}{ Education level } \\
\hline Illiterate & 0.169 & 0.027 & 0.117 & 0.221 & 0.157 & 1.579 & 284 & 0.037 & 0.010 & 0.018 & 0.056 & 0.264 & 1.755 & 825 \\
\hline Read and write & 0.125 & 0.064 & 0.000 & 0.250 & 0.509 & 1.460 & 38 & 0.049 & 0.026 & -0.003 & 0.101 & 0.537 & 1.007 & 83 \\
\hline Primary & 0.266 & 0.026 & 0.216 & 0.317 & 0.097 & 1.640 & 443 & 0.044 & 0.013 & 0.019 & 0.068 & 0.286 & 1.385 & 425 \\
\hline Preparatory & 0.251 & 0.023 & 0.206 & 0.297 & 0.091 & 1.635 & 538 & 0.044 & 0.009 & 0.026 & 0.063 & 0.214 & 1.435 & 742 \\
\hline General secondary & 0.224 & 0.023 & 0.177 & 0.270 & 0.105 & 1.372 & 412 & 0.115 & 0.017 & 0.081 & 0.149 & 0.150 & 1.388 & 506 \\
\hline Vocational secondary & 0.263 & 0.015 & 0.234 & 0.292 & 0.057 & 2.565 & 2,024 & 0.063 & 0.008 & 0.048 & 0.077 & 0.121 & 1.717 & 1,949 \\
\hline Post-secondary institute & 0.238 & 0.037 & 0.165 & 0.311 & 0.156 & 1.564 & 188 & 0.106 & 0.029 & 0.050 & 0.162 & 0.268 & 1.304 & 165 \\
\hline University and above & 0.318 & 0.020 & 0.279 & 0.357 & 0.062 & 2.083 & 1,014 & 0.152 & 0.016 & 0.120 & 0.183 & 0.105 & 1.943 & 982 \\
\hline \multicolumn{15}{|l|}{ Wealth quintile } \\
\hline Lowest & 0.226 & 0.021 & 0.185 & 0.267 & 0.092 & 2.387 & 798 & 0.039 & 0.008 & 0.023 & 0.056 & 0.216 & 1.846 & 989 \\
\hline Second & 0.240 & 0.018 & 0.205 & 0.274 & 0.073 & 1.850 & 928 & 0.068 & 0.010 & 0.048 & 0.087 & 0.146 & 1.623 & 1,118 \\
\hline Middle & 0.250 & 0.019 & 0.213 & 0.287 & 0.075 & 1.901 & 964 & 0.083 & 0.014 & 0.056 & 0.110 & 0.166 & 2.413 & 1,126 \\
\hline Fourth & 0.262 & 0.018 & 0.227 & 0.297 & 0.068 & 1.805 & 1,054 & 0.076 & 0.011 & 0.053 & 0.098 & 0.151 & 1.871 & 1,159 \\
\hline Highest & 0.322 & 0.019 & 0.285 & 0.359 & 0.059 & 2.135 & 1,197 & 0.119 & 0.011 & 0.099 & 0.140 & 0.088 & 1.244 & 1,288 \\
\hline
\end{tabular}


Table S17 Indicator: Proportion who have migrated and returned

\begin{tabular}{|c|c|c|c|c|c|c|c|c|c|c|c|c|c|c|}
\hline \multirow[b]{3}{*}{ Characteristic } & \multirow[b]{3}{*}{ Estimate } & \multirow[b]{3}{*}{ SE } & \multicolumn{3}{|c|}{ Male } & \multirow{3}{*}{$\begin{array}{l}\text { Design } \\
\text { effect }\end{array}$} & \multirow[b]{3}{*}{ Count } & \multirow[b]{3}{*}{ Estimate } & \multirow[b]{3}{*}{ SE } & \multirow{2}{*}{\multicolumn{2}{|c|}{$\begin{array}{l}\text { Female } \\
5 \% \text { C.I }\end{array}$}} & \multirow{3}{*}{$\begin{array}{l}\text { CV } \\
(\%)\end{array}$} & \multirow{3}{*}{$\begin{array}{c}\text { Design } \\
\text { effect }\end{array}$} & \multirow[b]{3}{*}{ Count } \\
\hline & & & \multicolumn{2}{|c|}{ 95\% C.I } & \multirow{2}{*}{$\begin{array}{l}\text { CV } \\
(\%)\end{array}$} & & & & & & & & & \\
\hline & & & Lower & Upper & & & & & & Lower & Upper & & & \\
\hline Urban-rural residence & & & & & & & & & & & & & & \\
\hline Urban & 0.022 & 0.005 & 0.011 & 0.033 & 0.247 & 1.886 & 1,283 & 0.010 & 0.003 & 0.003 & 0.017 & 0.345 & 1.457 & 1,343 \\
\hline Rural & 0.030 & 0.005 & 0.021 & 0.039 & 0.151 & 1.896 & 2,297 & 0.003 & 0.001 & 0.001 & 0.006 & 0.374 & 1.167 & 2,879 \\
\hline Informal urban areas & 0.022 & 0.008 & 0.006 & 0.038 & 0.373 & 1.386 & 402 & 0.007 & 0.004 & -0.001 & 0.015 & 0.610 & 1.142 & 516 \\
\hline Region & & & & & & & & & & & & & & \\
\hline Urban Governorates & 0.021 & 0.006 & 0.010 & 0.032 & 0.265 & 1.332 & 824 & 0.016 & 0.006 & 0.005 & 0.026 & 0.355 & 1.519 & 817 \\
\hline Rural Lower Egypt & 0.032 & 0.006 & 0.020 & 0.045 & 0.197 & 1.838 & 1,276 & 0.003 & 0.002 & -0.001 & 0.006 & 0.628 & 1.350 & 1,459 \\
\hline Urban Upper Egypt & 0.018 & 0.013 & -0.008 & 0.044 & 0.723 & 3.548 & 219 & 0.000 & 0.000 & 0.000 & 0.000 & 0.000 & 0.000 & 281 \\
\hline Rural Upper Egypt & 0.029 & 0.007 & 0.016 & 0.042 & 0.234 & 1.973 & 833 & 0.004 & 0.002 & 0.000 & 0.007 & 0.458 & 1.036 & 1,232 \\
\hline Frontier Governorates & 0.008 & 0.006 & -0.003 & 0.020 & 0.717 & 0.359 & 367 & 0.001 & 0.001 & -0.001 & 0.004 & 0.978 & 0.108 & 400 \\
\hline Age group & & & & & & & & & & & & & & \\
\hline $13-17$ years & 0.000 & 0.000 & 0.000 & 0.000 & 0.000 & 0.000 & 0.000 & 0.000 & 0.000 & 0.000 & 0.000 & 0.000 & 0.000 & \\
\hline $18-24$ years & 0.011 & 0.003 & 0.006 & 0.017 & 0.255 & 1.742 & 2,080 & 0.004 & 0.001 & 0.001 & 0.007 & 0.349 & 1.044 & 2,078 \\
\hline $25-29$ years & 0.047 & 0.008 & 0.032 & 0.063 & 0.166 & 1.787 & 1,108 & 0.009 & 0.003 & 0.002 & 0.016 & 0.379 & 1.649 & 1,456 \\
\hline $30-35$ years & 0.037 & 0.008 & 0.022 & 0.052 & 0.205 & 1.417 & 794 & 0.004 & 0.002 & 0.000 & 0.007 & 0.507 & 0.914 & 1,204 \\
\hline Illiterate & 0.013 & 0.008 & -0.002 & 0.028 & 0.572 & 1.223 & 252 & 0.000 & 0.000 & 0.000 & 0.000 & 0.000 & 0.000 & 790 \\
\hline Read and write & 0.000 & 0.000 & 0.000 & 0.000 & 0.000 & 0.000 & 35 & 0.000 & 0.000 & 0.000 & 0.000 & 0.000 & 0.000 & 82 \\
\hline Primary & 0.029 & 0.010 & 0.010 & 0.048 & 0.338 & 1.391 & 375 & 0.002 & 0.002 & -0.002 & 0.005 & 1.001 & 0.489 & 366 \\
\hline Preparatory & 0.033 & 0.011 & 0.011 & 0.055 & 0.344 & 1.464 & 318 & 0.004 & 0.003 & -0.001 & 0.009 & 0.709 & 0.813 & 508 \\
\hline General secondary & 0.034 & 0.017 & 0.000 & 0.068 & 0.502 & 1.383 & 139 & 0.011 & 0.007 & -0.003 & 0.025 & 0.662 & 0.657 & 166 \\
\hline Vocational secondary & 0.031 & 0.005 & 0.020 & 0.041 & 0.173 & 1.814 & 1,686 & 0.005 & 0.002 & 0.000 & 0.009 & 0.487 & 1.601 & 1,692 \\
\hline Post-secondary institute & 0.010 & 0.006 & -0.003 & 0.022 & 0.645 & 0.766 & 170 & 0.000 & 0.000 & 0.000 & 0.000 & 0.000 & 0.000 & 156 \\
\hline University and above & 0.024 & 0.006 & 0.012 & 0.035 & 0.248 & 1.704 & 1,007 & 0.013 & 0.004 & 0.005 & 0.021 & 0.300 & 1.202 & 975 \\
\hline Wealth quintile & & & & & & & & & & & & & & \\
\hline Lowest & 0.018 & 0.006 & 0.006 & 0.031 & 0.341 & 1.662 & 621 & 0.002 & 0.001 & -0.001 & 0.004 & 0.707 & 0.737 & 841 \\
\hline Second & 0.027 & 0.008 & 0.012 & 0.043 & 0.289 & 2.163 & 756 & 0.001 & 0.001 & -0.001 & 0.003 & 0.995 & 0.892 & 923 \\
\hline Middle & 0.027 & 0.007 & 0.013 & 0.041 & 0.265 & 1.575 & 759 & 0.007 & 0.004 & -0.001 & 0.015 & 0.580 & 1.852 & 943 \\
\hline Fourth & 0.018 & 0.005 & 0.008 & 0.028 & 0.289 & 1.404 & 860 & 0.003 & 0.002 & 0.000 & 0.007 & 0.580 & 0.871 & 957 \\
\hline Highest & 0.040 & 0.007 & 0.026 & 0.054 & 0.176 & 1.397 & 986 & 0.013 & 0.004 & 0.005 & 0.022 & 0.303 & 1.214 & 1,074 \\
\hline
\end{tabular}


Table S18 Indicator: Proportion of youth using internet

\begin{tabular}{|c|c|c|c|c|c|c|c|c|c|c|c|c|c|c|}
\hline \multirow[b]{3}{*}{ Characteristic } & \multirow[b]{3}{*}{ Estimate } & \multirow[b]{3}{*}{ SE } & \multicolumn{3}{|c|}{ Male } & \multirow{3}{*}{$\begin{array}{l}\text { Design } \\
\text { effect }\end{array}$} & \multirow[b]{3}{*}{ Count } & \multirow[b]{3}{*}{ Estimate } & \multirow[b]{3}{*}{ SE } & \multirow{2}{*}{\multicolumn{2}{|c|}{$\begin{array}{l}\text { Female } \\
95 \% \text { C.I }\end{array}$}} & \multirow{3}{*}{$\begin{array}{l}\text { CV } \\
(\%)\end{array}$} & \multirow{3}{*}{$\begin{array}{l}\text { Design } \\
\text { effect }\end{array}$} & \multirow[b]{3}{*}{ Count } \\
\hline & & & 95 & C.I & CV & & & & & & & & & \\
\hline & & & Lower & Upper & (\%) & & & & & Lower & Upper & & & \\
\hline \multicolumn{15}{|l|}{ Urban-rural residence } \\
\hline Urban & 0.449 & 0.030 & 0.390 & 0.508 & 0.067 & 6.202 & 1,619 & 0.384 & 0.028 & 0.329 & 0.439 & 0.073 & 5.094 & 1,690 \\
\hline Rural & 0.197 & 0.012 & 0.174 & 0.220 & 0.059 & 2.904 & 2,949 & 0.094 & 0.008 & 0.079 & 0.110 & 0.084 & 2.366 & 3,536 \\
\hline Informal urban areas & 0.256 & 0.034 & 0.188 & 0.323 & 0.134 & 3.300 & 492 & 0.184 & 0.034 & 0.118 & 0.250 & 0.183 & 4.067 & 603 \\
\hline \multicolumn{15}{|l|}{ Region } \\
\hline Urban Governorates & 0.515 & 0.035 & 0.446 & 0.584 & 0.068 & 5.298 & 1,025 & 0.450 & 0.033 & 0.385 & 0.515 & 0.073 & 4.239 & 1,038 \\
\hline Urban Lower Egypt & 0.367 & 0.035 & 0.299 & 0.435 & 0.095 & 3.289 & 572 & 0.248 & 0.031 & 0.187 & 0.309 & 0.126 & 3.242 & 639 \\
\hline Rural Lower Egypt & 0.196 & 0.016 & 0.163 & 0.228 & 0.084 & 3.068 & 1,616 & 0.108 & 0.011 & 0.086 & 0.131 & 0.105 & 2.163 & 1,807 \\
\hline Urban Upper Egypt & 0.203 & 0.045 & 0.115 & 0.292 & 0.222 & 5.696 & 289 & 0.200 & 0.050 & 0.101 & 0.299 & 0.253 & 6.759 & 349 \\
\hline Rural Upper Egypt & 0.202 & 0.017 & 0.168 & 0.235 & 0.084 & 2.755 & 1,104 & 0.080 & 0.011 & 0.059 & 0.102 & 0.137 & 2.600 & 1,500 \\
\hline Frontier Governorates & 0.161 & 0.030 & 0.102 & 0.221 & 0.189 & 0.700 & 454 & 0.167 & 0.035 & 0.099 & 0.235 & 0.207 & 0.833 & 496 \\
\hline \multicolumn{15}{|l|}{ Age group } \\
\hline $13-17$ years & 0.276 & 0.019 & 0.239 & 0.314 & 0.069 & 2.000 & 1,089 & 0.213 & 0.018 & 0.178 & 0.247 & 0.082 & 2.051 & 1,102 \\
\hline $18-24$ years & 0.313 & 0.015 & 0.283 & 0.342 & 0.048 & 2.441 & 2,079 & 0.233 & 0.015 & 0.203 & 0.263 & 0.065 & 2.568 & 2,075 \\
\hline $25-29$ years & 0.269 & 0.020 & 0.230 & 0.308 & 0.074 & 2.582 & 1,103 & 0.141 & 0.015 & 0.112 & 0.171 & 0.107 & 2.346 & 1,452 \\
\hline $30-35$ years & 0.204 & 0.022 & 0.161 & 0.246 & 0.106 & 2.495 & 789 & 0.120 & 0.015 & 0.091 & 0.150 & 0.124 & 2.052 & 1,200 \\
\hline \multicolumn{15}{|l|}{ Education level } \\
\hline Illiterate & 0.000 & 0.000 & 0.000 & 0.000 & 0.000 & 0.000 & 285 & 0.000 & 0.000 & 0.000 & 0.000 & 0.000 & 0.000 & 828 \\
\hline Read and write & 0.118 & 0.057 & 0.007 & 0.230 & 0.480 & 1.220 & 38 & 0.000 & 0.000 & 0.000 & 0.000 & 0.000 & 0.000 & 82 \\
\hline Primary & 0.064 & 0.017 & 0.030 & 0.097 & 0.269 & 2.420 & 453 & 0.012 & 0.006 & 0.000 & 0.024 & 0.509 & 1.213 & 437 \\
\hline Preparatory & 0.136 & 0.020 & 0.096 & 0.175 & 0.149 & 2.439 & 646 & 0.072 & 0.011 & 0.050 & 0.093 & 0.152 & 1.438 & 868 \\
\hline General secondary & 0.466 & 0.031 & 0.404 & 0.527 & 0.068 & 1.738 & 417 & 0.371 & 0.031 & 0.311 & 0.432 & 0.083 & 1.943 & 515 \\
\hline Vocational secondary & 0.160 & 0.012 & 0.136 & 0.184 & 0.076 & 2.466 & 2,022 & 0.102 & 0.012 & 0.078 & 0.125 & 0.116 & 2.690 & 1,950 \\
\hline Post-secondary institute & 0.393 & 0.045 & 0.305 & 0.481 & 0.114 & 1.724 & 188 & 0.294 & 0.043 & 0.211 & 0.378 & 0.145 & 1.331 & 165 \\
\hline University and above & 0.680 & 0.020 & 0.641 & 0.719 & 0.029 & 2.063 & 1,011 & 0.531 & 0.023 & 0.486 & 0.577 & 0.044 & 2.159 & 981 \\
\hline \multicolumn{15}{|l|}{ Wealth quintile } \\
\hline Lowest & 0.104 & 0.015 & 0.076 & 0.133 & 0.139 & 2.227 & 824 & 0.032 & 0.008 & 0.015 & 0.048 & 0.263 & 2.289 & 1,015 \\
\hline Second & 0.130 & 0.015 & 0.100 & 0.160 & 0.116 & 2.265 & 951 & 0.084 & 0.013 & 0.059 & 0.108 & 0.150 & 2.182 & 1,142 \\
\hline Middle & 0.219 & 0.019 & 0.181 & 0.257 & 0.089 & 2.248 & 986 & 0.108 & 0.014 & 0.081 & 0.135 & 0.128 & 2.003 & 1,163 \\
\hline Fourth & 0.323 & 0.021 & 0.281 & 0.364 & 0.066 & 2.318 & 1,080 & 0.222 & 0.021 & 0.182 & 0.263 & 0.092 & 2.501 & 1,190 \\
\hline Highest & 0.542 & 0.024 & 0.495 & 0.590 & 0.045 & 3.120 & 1,219 & 0.441 & 0.024 & 0.393 & 0.489 & 0.055 & 2.932 & 1,319 \\
\hline
\end{tabular}




\section{Table S19 Indicator: Proportion of youth participating in voluntary work}

\begin{tabular}{|c|c|c|c|c|c|c|c|c|c|c|c|c|c|c|}
\hline \multirow[b]{3}{*}{ Characteristic } & \multirow[b]{3}{*}{ Estimate } & \multirow[b]{3}{*}{ SE } & \multicolumn{3}{|c|}{ Male } & \multirow{3}{*}{$\begin{array}{c}\text { Design } \\
\text { effect }\end{array}$} & \multirow[b]{3}{*}{ Count } & \multirow[b]{3}{*}{ Estimate } & \multirow[b]{3}{*}{ SE } & \multicolumn{2}{|c|}{ Female } & \multirow{3}{*}{$\begin{array}{l}\text { CV } \\
(\%)\end{array}$} & \multirow{3}{*}{$\begin{array}{l}\text { Design } \\
\text { effect }\end{array}$} & \multirow[b]{3}{*}{ Count } \\
\hline & & & \multicolumn{2}{|c|}{ 95\% C.I } & \multirow{2}{*}{$\begin{array}{l}\text { CV } \\
(\%)\end{array}$} & & & & & \multicolumn{2}{|c|}{ 95\% C.I } & & & \\
\hline & & & Lower & Upper & & & & & & Lower & Upper & & & \\
\hline \multicolumn{15}{|l|}{ Urban-rural residence } \\
\hline Urban & 0.040 & 0.007 & 0.026 & 0.054 & 0.179 & 2.239 & 1,619 & 0.041 & 0.008 & 0.026 & 0.056 & 0.190 & 2.361 & 1,690 \\
\hline Rural & 0.033 & 0.005 & 0.022 & 0.043 & 0.164 & 3.055 & 2,949 & 0.017 & 0.003 & 0.011 & 0.022 & 0.161 & 1.429 & 3,536 \\
\hline Informal urban areas & 0.045 & 0.016 & 0.014 & 0.077 & 0.351 & 3.127 & 493 & 0.034 & 0.010 & 0.014 & 0.053 & 0.296 & 1.635 & 603 \\
\hline \multicolumn{15}{|l|}{ Region } \\
\hline Urban Governorates & 0.041 & 0.008 & 0.026 & 0.056 & 0.187 & 1.617 & 1,025 & 0.039 & 0.008 & 0.023 & 0.055 & 0.212 & 1.743 & 1,038 \\
\hline Urban Lower Egypt & 0.054 & 0.017 & 0.020 & 0.088 & 0.323 & 3.779 & 573 & 0.048 & 0.014 & 0.021 & 0.074 & 0.283 & 2.512 & 639 \\
\hline Rural Lower Egypt & 0.029 & 0.006 & 0.017 & 0.041 & 0.209 & 2.333 & 1,616 & 0.018 & 0.004 & 0.010 & 0.025 & 0.208 & 1.260 & 1,807 \\
\hline Urban Upper Egypt & 0.023 & 0.009 & 0.005 & 0.041 & 0.398 & 1.681 & 289 & 0.028 & 0.014 & 0.001 & 0.055 & 0.489 & 2.909 & 349 \\
\hline Rural Upper Egypt & 0.037 & 0.009 & 0.019 & 0.056 & 0.252 & 3.787 & 1,104 & 0.016 & 0.004 & 0.008 & 0.024 & 0.256 & 1.658 & 1,500 \\
\hline Frontier Governorates & 0.038 & 0.011 & 0.018 & 0.059 & 0.275 & 0.309 & 454 & 0.028 & 0.009 & 0.011 & 0.046 & 0.318 & 0.283 & 496 \\
\hline \multicolumn{15}{|l|}{ Age group } \\
\hline $13-17$ years & 0.032 & 0.007 & 0.018 & 0.045 & 0.224 & 1.803 & 1,089 & 0.026 & 0.005 & 0.017 & 0.035 & 0.182 & 0.993 & 1,102 \\
\hline $18-24$ years & 0.038 & 0.006 & 0.026 & 0.049 & 0.161 & 2.349 & 2,079 & 0.032 & 0.005 & 0.022 & 0.042 & 0.163 & 1.762 & 2,075 \\
\hline $25-29$ years & 0.040 & 0.007 & 0.026 & 0.054 & 0.178 & 1.718 & 1,103 & 0.016 & 0.004 & 0.008 & 0.023 & 0.240 & 1.151 & 1,452 \\
\hline $30-35$ years & 0.032 & 0.007 & 0.019 & 0.046 & 0.215 & 1.322 & 790 & 0.023 & 0.006 & 0.012 & 0.034 & 0.247 & 1.392 & 1,200 \\
\hline \multicolumn{15}{|l|}{ Education level } \\
\hline Illiterate & 0.009 & 0.007 & -0.004 & 0.023 & 0.749 & 1.653 & 285 & 0.001 & 0.001 & -0.001 & 0.004 & 0.994 & 0.864 & 828 \\
\hline Read and write & 0.023 & 0.023 & -0.022 & 0.067 & 0.997 & 0.904 & 38 & 0.000 & 0.000 & 0.000 & 0.000 & 0.000 & 0.000 & 82 \\
\hline Primary & 0.007 & 0.004 & -0.002 & 0.016 & 0.616 & 1.362 & 453 & 0.006 & 0.004 & -0.001 & 0.013 & 0.579 & 0.802 & 437 \\
\hline Preparatory & 0.016 & 0.007 & 0.003 & 0.030 & 0.428 & 2.113 & 646 & 0.011 & 0.004 & 0.004 & 0.018 & 0.338 & 1.003 & 868 \\
\hline General secondary & 0.056 & 0.015 & 0.026 & 0.087 & 0.274 & 1.955 & 417 & 0.046 & 0.009 & 0.027 & 0.065 & 0.206 & 0.984 & 515 \\
\hline Vocational secondary & 0.032 & 0.006 & 0.021 & 0.043 & 0.177 & 2.319 & 2,022 & 0.017 & 0.004 & 0.010 & 0.025 & 0.211 & 1.394 & 1,950 \\
\hline Post-secondary institute & 0.041 & 0.016 & 0.009 & 0.073 & 0.394 & 1.357 & 188 & 0.042 & 0.017 & 0.009 & 0.075 & 0.403 & 1.087 & 165 \\
\hline University and above & 0.068 & 0.010 & 0.048 & 0.088 & 0.148 & 1.833 & 1,012 & 0.064 & 0.010 & 0.045 & 0.084 & 0.153 & 1.595 & 981 \\
\hline \multicolumn{15}{|l|}{ Wealth quintile } \\
\hline Lowest & 0.020 & 0.005 & 0.009 & 0.031 & 0.272 & 1.502 & 824 & 0.008 & 0.003 & 0.003 & 0.013 & 0.341 & 0.896 & 1,015 \\
\hline Second & 0.022 & 0.005 & 0.012 & 0.033 & 0.242 & 1.477 & 951 & 0.022 & 0.006 & 0.011 & 0.034 & 0.252 & 1.551 & 1,142 \\
\hline Middle & 0.025 & 0.008 & 0.010 & 0.041 & 0.314 & 2.593 & 986 & 0.015 & 0.004 & 0.008 & 0.023 & 0.258 & 1.036 & 1,163 \\
\hline Fourth & 0.057 & 0.009 & 0.038 & 0.075 & 0.167 & 1.889 & 1,080 & 0.031 & 0.007 & 0.017 & 0.045 & 0.228 & 1.720 & 1,190 \\
\hline Highest & 0.051 & 0.008 & 0.034 & 0.068 & 0.166 & 1.948 & 1,220 & 0.046 & 0.008 & 0.031 & 0.061 & 0.165 & 1.608 & 1,319 \\
\hline
\end{tabular}


Table S20 Indicator: Proportion of youth participating in a group for social work

\begin{tabular}{|c|c|c|c|c|c|c|c|c|c|c|c|c|c|c|}
\hline \multirow[b]{3}{*}{ Characteristic } & \multirow[b]{3}{*}{ Estimate } & \multirow[b]{3}{*}{ SE } & \multirow{2}{*}{\multicolumn{2}{|c|}{$\begin{aligned} & \text { Male } \\
& 95 \% \text { C.I }\end{aligned}$}} & \multirow{3}{*}{$\begin{array}{l}\text { CV } \\
\text { (\%) }\end{array}$} & \multirow{3}{*}{$\begin{array}{l}\text { Design } \\
\text { effect }\end{array}$} & \multirow[b]{3}{*}{ Count } & \multirow[b]{3}{*}{ Estimate } & \multirow[b]{3}{*}{ SE } & \multirow{2}{*}{\multicolumn{2}{|c|}{$\begin{array}{l}\text { Female } \\
95 \% \text { C.I }\end{array}$}} & \multirow{3}{*}{$\begin{array}{l}\text { CV } \\
\text { (\%) }\end{array}$} & \multirow{3}{*}{$\begin{array}{c}\text { Design } \\
\text { effect }\end{array}$} & \multirow[b]{3}{*}{ Count } \\
\hline & & & & & & & & & & & & & & \\
\hline & & & Lower & Upper & & & & & & Lower & Upper & & & \\
\hline \multicolumn{15}{|l|}{ Urban-rural residence } \\
\hline Urban & 0.071 & 0.013 & 0.045 & 0.097 & 0.185 & 4.415 & 1,622 & 0.034 & 0.008 & 0.019 & 0.050 & 0.226 & 2.793 & 1,692 \\
\hline Rural & 0.032 & 0.004 & 0.023 & 0.041 & 0.139 & 2.151 & 2,957 & 0.008 & 0.002 & 0.005 & 0.012 & 0.216 & 1.261 & 3,546 \\
\hline Informal urban areas & 0.003 & 0.002 & -0.001 & 0.006 & 0.693 & 0.653 & 494 & 0.000 & 0.000 & 0.000 & 0.000 & 0.000 & 0.000 & 605 \\
\hline \multicolumn{15}{|l|}{ Region } \\
\hline Urban Governorates & 0.077 & 0.018 & 0.042 & 0.112 & 0.233 & 4.855 & 1,025 & 0.036 & 0.010 & 0.015 & 0.056 & 0.289 & 3.007 & 1,039 \\
\hline Urban Lower Egypt & 0.031 & 0.013 & 0.004 & 0.057 & 0.435 & 3.828 & 576 & 0.009 & 0.004 & 0.001 & 0.017 & 0.442 & 1.095 & 641 \\
\hline Rural Lower Egypt & 0.038 & 0.007 & 0.024 & 0.052 & 0.186 & 2.413 & 1,618 & 0.010 & 0.002 & 0.006 & 0.015 & 0.233 & 0.907 & 1,808 \\
\hline Urban Upper Egypt & 0.034 & 0.016 & 0.003 & 0.065 & 0.468 & 3.480 & 289 & 0.026 & 0.014 & -0.002 & 0.053 & 0.546 & 3.318 & 350 \\
\hline Rural Upper Egypt & 0.026 & 0.005 & 0.016 & 0.037 & 0.200 & 1.652 & 1,110 & 0.006 & 0.003 & 0.001 & 0.012 & 0.426 & 1.827 & 1,509 \\
\hline Frontier Governorates & 0.037 & 0.012 & 0.013 & 0.062 & 0.329 & 0.431 & 455 & 0.019 & 0.008 & 0.003 & 0.034 & 0.414 & 0.314 & 496 \\
\hline \multicolumn{15}{|l|}{ Age group } \\
\hline $13-17$ years & 0.048 & 0.009 & 0.030 & 0.066 & 0.191 & 2.039 & 1,091 & 0.019 & 0.005 & 0.010 & 0.028 & 0.248 & 1.332 & 1,105 \\
\hline $18-24$ years & 0.045 & 0.006 & 0.032 & 0.057 & 0.141 & 2.158 & 2,080 & 0.015 & 0.003 & 0.009 & 0.022 & 0.216 & 1.463 & 2,078 \\
\hline $25-29$ years & 0.034 & 0.007 & 0.020 & 0.047 & 0.198 & 1.772 & 1,108 & 0.011 & 0.004 & 0.004 & 0.019 & 0.340 & 1.653 & 1,456 \\
\hline $30-35$ years & 0.033 & 0.008 & 0.018 & 0.048 & 0.228 & 1.551 & 794 & 0.014 & 0.004 & 0.006 & 0.022 & 0.299 & 1.206 & 1,204 \\
\hline \multicolumn{15}{|l|}{ Education level } \\
\hline Illiterate & 0.000 & 0.000 & 0.000 & 0.000 & 0.000 & 0.000 & 288 & 0.000 & 0.000 & 0.000 & 0.000 & 0.000 & 0.000 & 831 \\
\hline Read and write & 0.000 & 0.000 & 0.000 & 0.000 & 0.000 & 0.000 & 38 & 0.000 & 0.000 & 0.000 & 0.000 & 0.000 & 0.000 & 83 \\
\hline Primary & 0.017 & 0.008 & 0.001 & 0.033 & 0.485 & 1.979 & 455 & 0.000 & 0.000 & 0.000 & 0.000 & 0.000 & 0.000 & 439 \\
\hline Preparatory & 0.023 & 0.008 & 0.007 & 0.038 & 0.354 & 2.028 & 646 & 0.005 & 0.002 & 0.000 & 0.009 & 0.511 & 0.990 & 872 \\
\hline General secondary & 0.097 & 0.017 & 0.063 & 0.131 & 0.179 & 1.498 & 417 & 0.037 & 0.010 & 0.017 & 0.056 & 0.271 & 1.352 & 516 \\
\hline Vocational secondary & 0.021 & 0.004 & 0.012 & 0.029 & 0.215 & 2.184 & 2,027 & 0.005 & 0.002 & 0.002 & 0.008 & 0.314 & 0.919 & 1,952 \\
\hline Post-secondary institute & 0.068 & 0.019 & 0.031 & 0.106 & 0.281 & 1.186 & 188 & 0.012 & 0.012 & -0.011 & 0.034 & 0.995 & 1.775 & 165 \\
\hline University and above & 0.089 & 0.011 & 0.067 & 0.111 & 0.126 & 1.775 & 1,014 & 0.047 & 0.010 & 0.029 & 0.066 & 0.201 & 1.998 & 982 \\
\hline \multicolumn{15}{|l|}{ Wealth quintile } \\
\hline Lowest & 0.018 & 0.006 & 0.006 & 0.029 & 0.321 & 1.821 & 829 & 0.000 & 0.000 & 0.000 & 0.001 & 1.004 & 0.235 & 1,021 \\
\hline Second & 0.021 & 0.005 & 0.011 & 0.032 & 0.249 & 1.516 & 954 & 0.006 & 0.003 & 0.001 & 0.012 & 0.422 & 1.247 & 1,145 \\
\hline Middle & 0.021 & 0.007 & 0.007 & 0.034 & 0.336 & 2.419 & 987 & 0.003 & 0.002 & -0.001 & 0.007 & 0.665 & 1.317 & 1,165 \\
\hline Fourth & 0.039 & 0.007 & 0.025 & 0.053 & 0.184 & 1.567 & 1,082 & 0.016 & 0.004 & 0.008 & 0.024 & 0.256 & 1.105 & 1,192 \\
\hline Highest & 0.093 & 0.012 & 0.069 & 0.117 & 0.132 & 2.356 & 1,221 & 0.044 & 0.009 & 0.027 & 0.060 & 0.196 & 2.113 & 1,320 \\
\hline
\end{tabular}


Table S21 Indicator: Proportion of youth who voted in any election

\begin{tabular}{|c|c|c|c|c|c|c|c|c|c|c|c|c|c|c|}
\hline \multirow[b]{2}{*}{ Characteristic } & \multirow[b]{2}{*}{ Estimate } & \multirow[b]{2}{*}{ SE } & \multicolumn{2}{|c|}{$\begin{array}{r}\text { Male } \\
95 \% \text { C.I }\end{array}$} & \multirow{2}{*}{$\begin{array}{l}\text { CV } \\
\text { (\%) }\end{array}$} & \multirow{2}{*}{$\begin{array}{c}\text { Design } \\
\text { effect }\end{array}$} & \multirow[b]{2}{*}{ Count } & \multirow[b]{2}{*}{ Estimate } & \multirow[b]{2}{*}{ SE } & \multicolumn{2}{|c|}{$\begin{array}{l}\text { Female } \\
95 \% \text { C.I }\end{array}$} & \multirow{2}{*}{$\begin{array}{l}\text { CV } \\
\text { (\%) }\end{array}$} & \multirow{2}{*}{$\begin{array}{c}\text { Design } \\
\text { effect }\end{array}$} & \multirow[b]{2}{*}{ Count } \\
\hline & & & Lower & Upper & & & & & & Lower & Upper & & & \\
\hline \multicolumn{15}{|l|}{ Urban-rural residence } \\
\hline Urban & 0.703 & 0.026 & 0.653 & 0.754 & 0.037 & 4.342 & 1,283 & 0.643 & 0.023 & 0.597 & 0.688 & 0.036 & 2.845 & 1,343 \\
\hline Rural & 0.699 & 0.013 & 0.673 & 0.725 & 0.019 & 2.215 & 2,297 & 0.535 & 0.016 & 0.503 & 0.567 & 0.030 & 2.743 & 2,879 \\
\hline Informal urban areas & 0.791 & 0.031 & 0.729 & 0.852 & 0.040 & 2.659 & 402 & 0.760 & 0.035 & 0.692 & 0.828 & 0.046 & 2.957 & 516 \\
\hline \multicolumn{15}{|l|}{ Region } \\
\hline Urban Governorates & 0.696 & 0.032 & 0.634 & 0.759 & 0.046 & 4.196 & 824 & 0.667 & 0.027 & 0.614 & 0.719 & 0.040 & 2.455 & 817 \\
\hline Urban Lower Egypt & 0.779 & 0.039 & 0.702 & 0.855 & 0.050 & 4.635 & 463 & 0.769 & 0.031 & 0.708 & 0.829 & 0.040 & 2.805 & 549 \\
\hline Rural Lower Egypt & 0.715 & 0.017 & 0.681 & 0.748 & 0.024 & 2.046 & 1,276 & 0.649 & 0.020 & 0.610 & 0.688 & 0.030 & 2.134 & 1,459 \\
\hline Urban Upper Egypt & 0.722 & 0.043 & 0.638 & 0.807 & 0.060 & 3.390 & 219 & 0.553 & 0.046 & 0.463 & 0.643 & 0.083 & 2.855 & 281 \\
\hline Rural Upper Egypt & 0.682 & 0.021 & 0.641 & 0.723 & 0.031 & 2.458 & 833 & 0.427 & 0.021 & 0.387 & 0.468 & 0.048 & 2.214 & 1,232 \\
\hline Frontier Governorates & 0.672 & 0.030 & 0.613 & 0.731 & 0.045 & 0.343 & 367 & 0.497 & 0.047 & 0.405 & 0.588 & 0.094 & 0.657 & 400 \\
\hline \multicolumn{15}{|l|}{ Age group } \\
\hline $13-17$ years & 0.000 & 0.000 & 0.000 & 0.000 & 0.000 & 0.000 & 0.000 & 0.000 & 0.000 & 0.000 & 0.000 & 0.000 & 0.000 & 0.000 \\
\hline $18-24$ years & 0.639 & 0.014 & 0.610 & 0.667 & 0.023 & 2.111 & 2,080 & 0.508 & 0.016 & 0.476 & 0.540 & 0.032 & 2.157 & 2,078 \\
\hline $25-29$ years & 0.785 & 0.017 & 0.751 & 0.819 & 0.022 & 2.320 & 1,108 & 0.664 & 0.017 & 0.631 & 0.697 & 0.025 & 1.580 & 1,456 \\
\hline $30-35$ years & 0.787 & 0.020 & 0.748 & 0.826 & 0.025 & 2.025 & 794 & 0.664 & 0.018 & 0.630 & 0.699 & 0.026 & 1.343 & 1,204 \\
\hline \multicolumn{15}{|l|}{ Education level } \\
\hline Illiterate & 0.547 & 0.039 & 0.471 & 0.624 & 0.071 & 1.707 & 252 & 0.374 & 0.024 & 0.328 & 0.421 & 0.063 & 1.509 & 790 \\
\hline Read and write & 0.756 & 0.078 & 0.602 & 0.910 & 0.104 & 1.240 & 35 & 0.613 & 0.056 & 0.502 & 0.723 & 0.092 & 0.894 & 82 \\
\hline Primary & 0.624 & 0.032 & 0.561 & 0.686 & 0.051 & 1.789 & 375 & 0.538 & 0.033 & 0.474 & 0.603 & 0.061 & 1.362 & 366 \\
\hline Preparatory & 0.651 & 0.031 & 0.590 & 0.712 & 0.048 & 1.549 & 318 & 0.461 & 0.025 & 0.412 & 0.511 & 0.055 & 1.128 & 508 \\
\hline General secondary & 0.502 & 0.049 & 0.405 & 0.599 & 0.098 & 1.512 & 139 & 0.487 & 0.046 & 0.396 & 0.578 & 0.095 & 1.170 & 166 \\
\hline Vocational secondary & 0.719 & 0.015 & 0.690 & 0.748 & 0.020 & 2.022 & 1,686 & 0.617 & 0.017 & 0.583 & 0.651 & 0.028 & 1.849 & 1,692 \\
\hline Post-secondary institute & 0.791 & 0.043 & 0.708 & 0.875 & 0.054 & 2.103 & 170 & 0.752 & 0.041 & 0.673 & 0.832 & 0.054 & 1.255 & 156 \\
\hline University and above & 0.796 & 0.018 & 0.761 & 0.831 & 0.022 & 2.230 & 1,007 & 0.752 & 0.019 & 0.714 & 0.789 & 0.026 & 1.948 & 975 \\
\hline \multicolumn{15}{|l|}{ Wealth quintile } \\
\hline Lowest & 0.631 & 0.024 & 0.583 & 0.679 & 0.039 & 1.942 & 621 & 0.438 & 0.024 & 0.391 & 0.484 & 0.054 & 1.854 & 841 \\
\hline Second & 0.672 & 0.022 & 0.629 & 0.715 & 0.033 & 2.010 & 756 & 0.559 & 0.024 & 0.512 & 0.605 & 0.042 & 1.900 & 923 \\
\hline Middle & 0.754 & 0.019 & 0.716 & 0.792 & 0.026 & 1.622 & 759 & 0.570 & 0.021 & 0.528 & 0.611 & 0.037 & 1.439 & 943 \\
\hline Fourth & 0.735 & 0.020 & 0.695 & 0.774 & 0.027 & 1.914 & 860 & 0.660 & 0.021 & 0.618 & 0.702 & 0.032 & 1.626 & 957 \\
\hline Highest & 0.743 & 0.020 & 0.704 & 0.781 & 0.026 & 2.155 & 986 & 0.705 & 0.019 & 0.668 & 0.741 & 0.026 & 1.603 & 1,074 \\
\hline
\end{tabular}


TABLE S23 Indicator: Proportion participating in sports club/youth center

\begin{tabular}{|c|c|c|c|c|c|c|c|c|c|c|c|c|c|c|}
\hline \multirow[b]{3}{*}{ Characteristic } & \multirow[b]{3}{*}{ Estimate } & \multirow[b]{3}{*}{ SE } & \multicolumn{3}{|c|}{ Male } & \multirow{3}{*}{$\begin{array}{l}\text { Design } \\
\text { effect }\end{array}$} & \multirow[b]{3}{*}{ Count } & \multirow[b]{3}{*}{ Estimate } & \multirow[b]{3}{*}{ SE } & \multirow{2}{*}{\multicolumn{2}{|c|}{$\begin{array}{l}\text { Female } \\
95 \% \text { C.I }\end{array}$}} & \multirow{3}{*}{$\begin{array}{l}\text { CV } \\
\text { (\%) }\end{array}$} & \multirow{3}{*}{$\begin{array}{c}\text { Design } \\
\text { effect }\end{array}$} & \multirow[b]{3}{*}{ Count } \\
\hline & & & 95 & C.I & CV & & & & & & & & & \\
\hline & & & Lower & Upper & (\%) & & & & & Lower & Upper & & & \\
\hline \multicolumn{15}{|l|}{ Urban-rural residence } \\
\hline Urban & 0.058 & 0.012 & 0.034 & 0.083 & 0.214 & 4.784 & 1,618 & 0.020 & 0.006 & 0.008 & 0.031 & 0.298 & 2.727 & 1,690 \\
\hline Rural & 0.022 & 0.004 & 0.014 & 0.029 & 0.181 & 2.408 & 2,946 & 0.003 & 0.001 & 0.001 & 0.005 & 0.380 & 1.382 & 3,531 \\
\hline Informal urban areas & 0.000 & 0.000 & 0.000 & 0.000 & 0.000 & 0.000 & 493 & 0.000 & 0.000 & 0.000 & 0.000 & 0.000 & 0.000 & 603 \\
\hline \multicolumn{15}{|l|}{ Region } \\
\hline Urban Governorates & 0.066 & 0.018 & 0.031 & 0.101 & 0.271 & 5.601 & 1,024 & 0.023 & 0.008 & 0.007 & 0.039 & 0.352 & 2.805 & 1,038 \\
\hline Urban Lower Egypt & 0.021 & 0.010 & 0.002 & 0.040 & 0.449 & 2.772 & 573 & 0.000 & 0.000 & 0.000 & 0.000 & 0.000 & 0.000 & 639 \\
\hline Rural Lower Egypt & 0.028 & 0.007 & 0.015 & 0.041 & 0.232 & 2.763 & 1,614 & 0.004 & 0.002 & 0.001 & 0.007 & 0.408 & 0.999 & 1,804 \\
\hline Urban Upper Egypt & 0.024 & 0.013 & -0.002 & 0.049 & 0.544 & 3.233 & 289 & 0.016 & 0.010 & -0.004 & 0.036 & 0.650 & 2.864 & 349 \\
\hline Rural Upper Egypt & 0.014 & 0.004 & 0.007 & 0.021 & 0.253 & 1.421 & 1,103 & 0.002 & 0.002 & -0.001 & 0.006 & 0.745 & 2.000 & 1,498 \\
\hline Frontier Governorates & 0.034 & 0.013 & 0.009 & 0.059 & 0.371 & 0.496 & 454 & 0.015 & 0.008 & 0.000 & 0.030 & 0.504 & 0.376 & 496 \\
\hline \multicolumn{15}{|l|}{ Age group } \\
\hline $13-17$ years & 0.046 & 0.009 & 0.029 & 0.063 & 0.190 & 1.903 & 1,089 & 0.015 & 0.004 & 0.006 & 0.024 & 0.293 & 1.458 & 1,102 \\
\hline $18-24$ years & 0.036 & 0.006 & 0.024 & 0.047 & 0.167 & 2.394 & 2,076 & 0.006 & 0.002 & 0.001 & 0.011 & 0.397 & 1.899 & 2,072 \\
\hline $25-29$ years & 0.020 & 0.005 & 0.009 & 0.031 & 0.273 & 1.955 & 1,102 & 0.005 & 0.003 & 0.000 & 0.011 & 0.552 & 2.062 & 1,450 \\
\hline $30-35$ years & 0.014 & 0.005 & 0.005 & 0.023 & 0.337 & 1.392 & 790 & 0.004 & 0.002 & 0.000 & 0.009 & 0.468 & 0.951 & 1,200 \\
\hline \multicolumn{15}{|l|}{ Education level } \\
\hline Illiterate & 0.000 & 0.000 & 0.000 & 0.000 & 0.000 & 0.000 & 284 & 0.000 & 0.000 & 0.000 & 0.000 & 0.000 & 0.000 & 828 \\
\hline Read and write & 0.000 & 0.000 & 0.000 & 0.000 & 0.000 & 0.000 & 38 & 0.000 & 0.000 & 0.000 & 0.000 & 0.000 & 0.000 & 82 \\
\hline Primary & 0.017 & 0.008 & 0.001 & 0.033 & 0.486 & 1.987 & 452 & 0.000 & 0.000 & 0.000 & 0.000 & 0.000 & 0.000 & 437 \\
\hline Preparatory & 0.021 & 0.008 & 0.006 & 0.037 & 0.373 & 2.116 & 646 & 0.003 & 0.002 & 0.000 & 0.007 & 0.580 & 0.849 & 867 \\
\hline General secondary & 0.090 & 0.016 & 0.058 & 0.121 & 0.180 & 1.396 & 417 & 0.029 & 0.009 & 0.011 & 0.047 & 0.320 & 1.486 & 515 \\
\hline Vocational secondary & 0.015 & 0.004 & 0.008 & 0.023 & 0.259 & 2.356 & 2,021 & 0.002 & 0.001 & 0.000 & 0.004 & 0.538 & 1.104 & 1,947 \\
\hline Post-secondary institute & 0.038 & 0.015 & 0.007 & 0.068 & 0.408 & 1.335 & 188 & 0.000 & 0.000 & 0.000 & 0.000 & 0.000 & 0.000 & 165 \\
\hline University and above & 0.058 & 0.010 & 0.038 & 0.077 & 0.176 & 2.157 & 1,011 & 0.020 & 0.007 & 0.006 & 0.033 & 0.347 & 2.378 & 981 \\
\hline \multicolumn{15}{|l|}{ Wealth quintile } \\
\hline Lowest & 0.013 & 0.005 & 0.003 & 0.023 & 0.405 & 2.064 & 823 & 0.000 & 0.000 & 0.000 & 0.001 & 1.004 & 0.235 & 1,015 \\
\hline Second & 0.011 & 0.003 & 0.004 & 0.018 & 0.313 & 1.227 & 950 & 0.003 & 0.002 & 0.000 & 0.006 & 0.583 & 1.063 & 1,141 \\
\hline Middle & 0.016 & 0.006 & 0.003 & 0.029 & 0.404 & 2.714 & 985 & 0.000 & 0.000 & 0.000 & 0.001 & 1.002 & 0.489 & 1,163 \\
\hline Fourth & 0.032 & 0.007 & 0.018 & 0.045 & 0.218 & 1.758 & 1,080 & 0.005 & 0.002 & 0.001 & 0.010 & 0.421 & 0.991 & 1,190 \\
\hline Highest & 0.071 & 0.011 & 0.049 & 0.093 & 0.157 & 2.469 & 1,219 & 0.025 & 0.007 & 0.011 & 0.040 & 0.292 & 2.648 & 1,315 \\
\hline
\end{tabular}


TABLE S23 Indicator: Proportion agreeing that educating boys is more important than educating girls

\begin{tabular}{|c|c|c|c|c|c|c|c|c|c|c|c|c|c|c|}
\hline \multirow[b]{3}{*}{ Characteristic } & \multirow[b]{3}{*}{ Estimate } & \multirow[b]{3}{*}{ SE } & \multirow{2}{*}{\multicolumn{2}{|c|}{$\begin{array}{l}\text { Male } \\
\text { 95\% C.I }\end{array}$}} & \multirow{3}{*}{$\begin{array}{l}\text { CV } \\
(\%)\end{array}$} & \multirow{3}{*}{$\begin{array}{c}\text { Design } \\
\text { effect }\end{array}$} & \multirow[b]{3}{*}{ Count } & \multirow[b]{3}{*}{ Estimate } & \multirow[b]{3}{*}{ SE } & \multirow{2}{*}{\multicolumn{2}{|c|}{$\begin{array}{l}\text { Female } \\
95 \% \text { C.I }\end{array}$}} & \multirow{3}{*}{$\begin{array}{l}\text { CV } \\
(\%)\end{array}$} & \multirow{3}{*}{$\begin{array}{c}\text { Design } \\
\text { effect }\end{array}$} & \multirow[b]{3}{*}{ Count } \\
\hline & & & & & & & & & & & & & & \\
\hline & & & Lower & Upper & & & & & & Lower & Upper & & & \\
\hline \multicolumn{15}{|l|}{ Urban-rural residence } \\
\hline Urban & 0.414 & 0.023 & 0.370 & 0.459 & 0.054 & 3.536 & 1,621 & 0.210 & 0.017 & 0.177 & 0.243 & 0.079 & 2.559 & 1,690 \\
\hline Rural & 0.316 & 0.015 & 0.287 & 0.346 & 0.047 & 3.482 & 2,951 & 0.217 & 0.012 & 0.194 & 0.240 & 0.055 & 2.712 & 3,544 \\
\hline Informal urban areas & 0.350 & 0.040 & 0.270 & 0.429 & 0.115 & 3.827 & 493 & 0.207 & 0.030 & 0.148 & 0.266 & 0.145 & 2.950 & 605 \\
\hline \multicolumn{15}{|l|}{ Region } \\
\hline Urban Governorates & 0.488 & 0.026 & 0.438 & 0.539 & 0.053 & 2.855 & 1,025 & 0.258 & 0.023 & 0.213 & 0.302 & 0.087 & 2.564 & 1,037 \\
\hline Urban Lower Egypt & 0.279 & 0.032 & 0.217 & 0.341 & 0.113 & 3.146 & 574 & 0.185 & 0.027 & 0.133 & 0.238 & 0.144 & 2.952 & 641 \\
\hline Rural Lower Egypt & 0.252 & 0.017 & 0.219 & 0.286 & 0.067 & 2.710 & 1,616 & 0.156 & 0.013 & 0.131 & 0.181 & 0.082 & 2.021 & 1,808 \\
\hline Urban Upper Egypt & 0.350 & 0.045 & 0.262 & 0.438 & 0.128 & 3.970 & 289 & 0.134 & 0.019 & 0.096 & 0.171 & 0.144 & 1.354 & 350 \\
\hline Rural Upper Egypt & 0.382 & 0.024 & 0.335 & 0.430 & 0.063 & 3.772 & 1,106 & 0.273 & 0.019 & 0.237 & 0.309 & 0.068 & 2.742 & 1,507 \\
\hline Frontier Governorates & 0.494 & 0.047 & 0.402 & 0.587 & 0.095 & 0.907 & 455 & 0.325 & 0.043 & 0.241 & 0.409 & 0.132 & 0.807 & 496 \\
\hline \multicolumn{15}{|l|}{ Age group } \\
\hline $13-17$ years & 0.362 & 0.018 & 0.327 & 0.398 & 0.050 & 1.581 & 1,089 & 0.173 & 0.014 & 0.147 & 0.200 & 0.078 & 1.436 & 1,105 \\
\hline $18-24$ years & 0.331 & 0.015 & 0.302 & 0.360 & 0.045 & 2.278 & 2,078 & 0.210 & 0.013 & 0.183 & 0.236 & 0.064 & 2.166 & 2,078 \\
\hline $25-29$ years & 0.348 & 0.020 & 0.308 & 0.388 & 0.058 & 2.364 & 1,105 & 0.238 & 0.013 & 0.212 & 0.264 & 0.056 & 1.189 & 1,453 \\
\hline $30-35$ years & 0.382 & 0.023 & 0.337 & 0.427 & 0.060 & 1.919 & 793 & 0.240 & 0.015 & 0.210 & 0.269 & 0.063 & 1.203 & 1,203 \\
\hline \multicolumn{15}{|l|}{ Education level } \\
\hline Illiterate & 0.416 & 0.040 & 0.337 & 0.494 & 0.097 & 2.078 & 285 & 0.319 & 0.022 & 0.277 & 0.362 & 0.068 & 1.428 & 831 \\
\hline Read and write & 0.469 & 0.091 & 0.289 & 0.648 & 0.195 & 1.320 & 38 & 0.359 & 0.059 & 0.244 & 0.474 & 0.163 & 1.006 & 83 \\
\hline Primary & 0.394 & 0.027 & 0.341 & 0.448 & 0.069 & 1.537 & 453 & 0.368 & 0.029 & 0.310 & 0.426 & 0.080 & 1.420 & 437 \\
\hline Preparatory & 0.414 & 0.024 & 0.366 & 0.462 & 0.059 & 1.713 & 645 & 0.258 & 0.020 & 0.218 & 0.298 & 0.079 & 1.771 & 871 \\
\hline General secondary & 0.276 & 0.025 & 0.226 & 0.326 & 0.092 & 1.421 & 417 & 0.110 & 0.017 & 0.077 & 0.143 & 0.151 & 1.355 & 516 \\
\hline Vocational secondary & 0.344 & 0.015 & 0.314 & 0.374 & 0.045 & 2.378 & 2,027 & 0.192 & 0.012 & 0.169 & 0.216 & 0.063 & 1.679 & 1,952 \\
\hline Post-secondary institute & 0.352 & 0.042 & 0.271 & 0.434 & 0.118 & 1.546 & 188 & 0.135 & 0.029 & 0.078 & 0.192 & 0.215 & 1.104 & 165 \\
\hline University and above & 0.304 & 0.022 & 0.261 & 0.348 & 0.073 & 2.677 & 1,012 & 0.139 & 0.014 & 0.111 & 0.168 & 0.104 & 1.730 & 981 \\
\hline \multicolumn{15}{|l|}{ Wealth quintile } \\
\hline Lowest & 0.410 & 0.023 & 0.364 & 0.456 & 0.057 & 2.209 & 826 & 0.283 & 0.020 & 0.243 & 0.322 & 0.071 & 1.982 & 1,021 \\
\hline Second & 0.339 & 0.022 & 0.297 & 0.382 & 0.064 & 2.313 & 951 & 0.235 & 0.017 & 0.201 & 0.269 & 0.074 & 1.785 & 1,144 \\
\hline Middle & 0.348 & 0.021 & 0.307 & 0.390 & 0.060 & 1.969 & 986 & 0.211 & 0.016 & 0.180 & 0.241 & 0.074 & 1.471 & 1,164 \\
\hline Fourth & 0.359 & 0.020 & 0.320 & 0.398 & 0.055 & 1.949 & 1,081 & 0.222 & 0.017 & 0.188 & 0.255 & 0.077 & 1.734 & 1,192 \\
\hline Highest & 0.303 & 0.021 & 0.262 & 0.345 & 0.069 & 2.743 & 1,221 & 0.135 & 0.013 & 0.109 & 0.160 & 0.096 & 1.724 & 1,318 \\
\hline
\end{tabular}


Table S24 Indicator: Proportion agreeing that a woman has the right to ask for a divorce

\begin{tabular}{|c|c|c|c|c|c|c|c|c|c|c|c|c|c|c|}
\hline \multirow[b]{3}{*}{ Characteristic } & \multirow[b]{3}{*}{ Estimate } & \multirow[b]{3}{*}{ SE } & \multirow{2}{*}{\multicolumn{2}{|c|}{$\begin{array}{r}\text { Male } \\
95 \% \text { C.I }\end{array}$}} & \multirow{3}{*}{$\begin{array}{l}\text { CV } \\
(\%)\end{array}$} & \multirow{3}{*}{$\begin{array}{c}\text { Design } \\
\text { effect }\end{array}$} & \multirow[b]{3}{*}{ Count } & \multirow[b]{3}{*}{ Estimate } & \multirow[b]{3}{*}{ SE } & \multirow{2}{*}{\multicolumn{2}{|c|}{$\begin{array}{l}\text { Female } \\
95 \% \text { C.I }\end{array}$}} & \multirow{3}{*}{$\begin{array}{l}\text { CV } \\
(\%)\end{array}$} & \multirow{3}{*}{$\begin{array}{c}\text { Design } \\
\text { effect }\end{array}$} & \multirow[b]{3}{*}{ Count } \\
\hline & & & & & & & & & & & & & & \\
\hline & & & Lower & Upper & & & & & & Lower & Upper & & & \\
\hline \multicolumn{15}{|l|}{ Urban-rural residence } \\
\hline Urban & 0.609 & 0.023 & 0.564 & 0.654 & 0.037 & 3.688 & 1,621 & 0.828 & 0.015 & 0.798 & 0.857 & 0.018 & 2.409 & 1,690 \\
\hline Rural & 0.638 & 0.017 & 0.605 & 0.672 & 0.027 & 4.297 & 2,951 & 0.807 & 0.011 & 0.785 & 0.829 & 0.014 & 2.613 & 3,544 \\
\hline Informal urban areas & 0.629 & 0.041 & 0.549 & 0.710 & 0.065 & 3.820 & 493 & 0.735 & 0.027 & 0.682 & 0.788 & 0.037 & 2.012 & 605 \\
\hline \multicolumn{15}{|l|}{ Region } \\
\hline Urban Governorates & 0.658 & 0.022 & 0.615 & 0.700 & 0.033 & 2.228 & 1,025 & 0.842 & 0.016 & 0.810 & 0.874 & 0.019 & 1.879 & 1,037 \\
\hline Urban Lower Egypt & 0.614 & 0.047 & 0.522 & 0.706 & 0.076 & 5.855 & 574 & 0.773 & 0.029 & 0.717 & 0.829 & 0.037 & 2.905 & 641 \\
\hline Rural Lower Egypt & 0.626 & 0.023 & 0.581 & 0.672 & 0.037 & 4.119 & 1,616 & 0.821 & 0.015 & 0.793 & 0.850 & 0.018 & 2.368 & 1,808 \\
\hline Urban Upper Egypt & 0.527 & 0.047 & 0.435 & 0.620 & 0.089 & 4.000 & 289 & 0.768 & 0.031 & 0.706 & 0.829 & 0.041 & 2.312 & 350 \\
\hline Rural Upper Egypt & 0.659 & 0.026 & 0.608 & 0.710 & 0.039 & 4.542 & 1,106 & 0.797 & 0.017 & 0.764 & 0.831 & 0.022 & 2.896 & 1,507 \\
\hline Frontier Governorates & 0.457 & 0.045 & 0.370 & 0.545 & 0.097 & 0.817 & 455 & 0.682 & 0.037 & 0.609 & 0.755 & 0.055 & 0.617 & 496 \\
\hline \multicolumn{15}{|l|}{ Age group } \\
\hline $13-17$ years & 0.592 & 0.019 & 0.555 & 0.629 & 0.032 & 1.620 & 1,089 & 0.809 & 0.016 & 0.778 & 0.839 & 0.019 & 1.754 & 1,105 \\
\hline $18-24$ years & 0.634 & 0.017 & 0.602 & 0.667 & 0.026 & 2.737 & 2,078 & 0.814 & 0.012 & 0.791 & 0.837 & 0.014 & 1.806 & 2,078 \\
\hline $25-29$ years & 0.634 & 0.021 & 0.592 & 0.676 & 0.034 & 2.561 & 1,105 & 0.803 & 0.013 & 0.778 & 0.829 & 0.016 & 1.323 & 1,453 \\
\hline $30-35$ years & 0.653 & 0.023 & 0.608 & 0.698 & 0.035 & 1.984 & 793 & 0.786 & 0.014 & 0.758 & 0.814 & 0.018 & 1.165 & 1,203 \\
\hline \multicolumn{15}{|l|}{ Education level } \\
\hline Illiterate & 0.475 & 0.044 & 0.388 & 0.563 & 0.093 & 2.476 & 285 & 0.691 & 0.022 & 0.648 & 0.733 & 0.031 & 1.453 & 831 \\
\hline Read and write & 0.649 & 0.084 & 0.484 & 0.814 & 0.129 & 1.219 & 38 & 0.694 & 0.059 & 0.578 & 0.810 & 0.085 & 1.106 & 83 \\
\hline Primary & 0.576 & 0.030 & 0.517 & 0.634 & 0.052 & 1.774 & 453 & 0.791 & 0.024 & 0.744 & 0.838 & 0.030 & 1.303 & 437 \\
\hline Preparatory & 0.585 & 0.027 & 0.532 & 0.637 & 0.046 & 2.072 & 645 & 0.799 & 0.019 & 0.763 & 0.836 & 0.023 & 1.751 & 871 \\
\hline General secondary & 0.691 & 0.024 & 0.643 & 0.738 & 0.035 & 1.216 & 417 & 0.853 & 0.017 & 0.820 & 0.886 & 0.020 & 1.115 & 516 \\
\hline Vocational secondary & 0.623 & 0.017 & 0.589 & 0.656 & 0.028 & 2.840 & 2,027 & 0.814 & 0.012 & 0.790 & 0.838 & 0.015 & 1.731 & 1,952 \\
\hline Post-secondary institute & 0.689 & 0.046 & 0.598 & 0.780 & 0.067 & 2.043 & 188 & 0.849 & 0.029 & 0.791 & 0.906 & 0.035 & 1.021 & 165 \\
\hline University and above & 0.697 & 0.019 & 0.659 & 0.735 & 0.028 & 2.037 & 1,012 & 0.857 & 0.014 & 0.829 & 0.884 & 0.016 & 1.535 & 981 \\
\hline \multicolumn{15}{|l|}{ Wealth quintile } \\
\hline Lowest & 0.562 & 0.025 & 0.513 & 0.610 & 0.044 & 2.487 & 826 & 0.761 & 0.017 & 0.729 & 0.794 & 0.022 & 1.513 & 1,021 \\
\hline Second & 0.601 & 0.023 & 0.556 & 0.646 & 0.038 & 2.449 & 951 & 0.803 & 0.014 & 0.775 & 0.830 & 0.018 & 1.351 & 1,144 \\
\hline Middle & 0.639 & 0.021 & 0.597 & 0.680 & 0.033 & 1.971 & 986 & 0.807 & 0.015 & 0.777 & 0.837 & 0.019 & 1.524 & 1,164 \\
\hline Fourth & 0.610 & 0.022 & 0.566 & 0.654 & 0.037 & 2.413 & 1,081 & 0.796 & 0.015 & 0.766 & 0.827 & 0.019 & 1.490 & 1,192 \\
\hline Highest & 0.710 & 0.018 & 0.674 & 0.746 & 0.026 & 2.172 & 1,221 & 0.851 & 0.013 & 0.825 & 0.877 & 0.016 & 1.681 & 1,318 \\
\hline
\end{tabular}


Table S25 Indicator: Proportion agreeing that a man is justified in beating his wife when she argues with him

\begin{tabular}{|c|c|c|c|c|c|c|c|c|c|c|c|c|c|c|}
\hline \multirow[b]{3}{*}{ Characteristic } & \multirow[b]{3}{*}{ Estimate } & \multirow[b]{3}{*}{ SE } & \multicolumn{3}{|c|}{ Male } & \multirow{3}{*}{$\begin{array}{c}\text { Design } \\
\text { effect }\end{array}$} & \multirow[b]{3}{*}{ Count } & \multirow[b]{3}{*}{ Estimate } & \multirow[b]{3}{*}{ SE } & \multirow{2}{*}{\multicolumn{2}{|c|}{$\begin{array}{l}\text { Female } \\
\% \text { C.I }\end{array}$}} & \multirow{3}{*}{$\begin{array}{l}\text { CV } \\
\text { (\%) }\end{array}$} & \multirow{3}{*}{$\begin{array}{c}\text { Design } \\
\text { effect }\end{array}$} & \multirow[b]{3}{*}{ Count } \\
\hline & & & 95 & C.I & CV & & & & & & & & & \\
\hline & & & Lower & Upper & (\%) & & & & & Lower & Upper & & & \\
\hline \multicolumn{15}{|l|}{ Urban-rural residence } \\
\hline Urban & 0.203 & 0.019 & 0.165 & 0.240 & 0.095 & 3.837 & 1,621 & 0.119 & 0.018 & 0.083 & 0.154 & 0.151 & 4.711 & 1,690 \\
\hline Rural & 0.263 & 0.017 & 0.229 & 0.296 & 0.065 & 5.061 & 2,951 & 0.158 & 0.013 & 0.133 & 0.184 & 0.082 & 4.162 & 3,544 \\
\hline Informal urban areas & 0.146 & 0.023 & 0.101 & 0.191 & 0.158 & 2.261 & 493 & 0.095 & 0.020 & 0.056 & 0.134 & 0.209 & 2.449 & 605 \\
\hline \multicolumn{15}{|l|}{ Region } \\
\hline Urban Governorates & 0.183 & 0.020 & 0.143 & 0.222 & 0.110 & 2.888 & 1,025 & 0.119 & 0.018 & 0.083 & 0.154 & 0.151 & 2.982 & 1,037 \\
\hline Urban Lower Egypt & 0.171 & 0.028 & 0.115 & 0.226 & 0.166 & 3.596 & 574 & 0.078 & 0.017 & 0.046 & 0.111 & 0.213 & 2.413 & 641 \\
\hline Rural Lower Egypt & 0.225 & 0.020 & 0.185 & 0.265 & 0.090 & 4.213 & 1,616 & 0.091 & 0.009 & 0.073 & 0.108 & 0.099 & 1.600 & 1,808 \\
\hline Urban Upper Egypt & 0.215 & 0.043 & 0.130 & 0.301 & 0.202 & 5.066 & 289 & 0.140 & 0.048 & 0.045 & 0.234 & 0.344 & 8.183 & 350 \\
\hline Rural Upper Egypt & 0.302 & 0.029 & 0.245 & 0.358 & 0.095 & 6.013 & 1,106 & 0.222 & 0.023 & 0.176 & 0.268 & 0.105 & 4.999 & 1,507 \\
\hline Frontier Governorates & 0.351 & 0.052 & 0.249 & 0.454 & 0.149 & 1.230 & 455 & 0.267 & 0.051 & 0.166 & 0.367 & 0.193 & 1.302 & 496 \\
\hline \multicolumn{15}{|l|}{ Age group } \\
\hline 13-17 years & 0.268 & 0.018 & 0.232 & 0.304 & 0.068 & 1.877 & 1,089 & 0.122 & 0.013 & 0.096 & 0.149 & 0.110 & 1.905 & 1,105 \\
\hline 18-24 years & 0.238 & 0.014 & 0.209 & 0.266 & 0.060 & 2.634 & 2,078 & 0.142 & 0.012 & 0.118 & 0.167 & 0.087 & 2.507 & 2,078 \\
\hline 25-29 years & 0.205 & 0.016 & 0.173 & 0.238 & 0.080 & 2.144 & 1,105 & 0.151 & 0.012 & 0.126 & 0.175 & 0.082 & 1.489 & 1,453 \\
\hline $30-35$ years & 0.219 & 0.021 & 0.177 & 0.261 & 0.098 & 2.335 & 793 & 0.145 & 0.014 & 0.117 & 0.173 & 0.099 & 1.598 & 1,203 \\
\hline \multicolumn{15}{|l|}{ Education level } \\
\hline Illiterate & 0.208 & 0.031 & 0.146 & 0.269 & 0.151 & 1.865 & 285 & 0.222 & 0.023 & 0.176 & 0.268 & 0.106 & 2.118 & 831 \\
\hline Read and write & 0.148 & 0.067 & 0.016 & 0.280 & 0.453 & 1.408 & 38 & 0.165 & 0.042 & 0.082 & 0.248 & 0.255 & 0.873 & 83 \\
\hline Primary & 0.270 & 0.027 & 0.217 & 0.323 & 0.099 & 1.785 & 453 & 0.203 & 0.023 & 0.157 & 0.249 & 0.115 & 1.274 & 437 \\
\hline Preparatory & 0.321 & 0.026 & 0.271 & 0.372 & 0.080 & 2.107 & 645 & 0.171 & 0.021 & 0.129 & 0.212 & 0.124 & 2.553 & 871 \\
\hline General secondary & 0.182 & 0.021 & 0.140 & 0.223 & 0.116 & 1.317 & 417 & 0.083 & 0.013 & 0.057 & 0.110 & 0.161 & 1.134 & 516 \\
\hline Vocational secondary & 0.246 & 0.017 & 0.213 & 0.279 & 0.069 & 3.441 & 2,027 & 0.117 & 0.011 & 0.097 & 0.138 & 0.090 & 1.904 & 1,952 \\
\hline Post-secondary institute & 0.201 & 0.032 & 0.137 & 0.265 & 0.161 & 1.335 & 188 & 0.140 & 0.036 & 0.069 & 0.211 & 0.258 & 1.648 & 165 \\
\hline University and above & 0.174 & 0.016 & 0.143 & 0.204 & 0.089 & 1.929 & 1,012 & 0.106 & 0.015 & 0.077 & 0.134 & 0.138 & 2.241 & 981 \\
\hline \multicolumn{15}{|l|}{ Wealth quintile } \\
\hline Lowest & 0.276 & 0.025 & 0.227 & 0.325 & 0.090 & 3.029 & 826 & 0.208 & 0.024 & 0.160 & 0.256 & 0.117 & 3.611 & 1,021 \\
\hline Second & 0.245 & 0.024 & 0.197 & 0.292 & 0.099 & 3.512 & 951 & 0.131 & 0.014 & 0.102 & 0.159 & 0.111 & 1.963 & 1,144 \\
\hline Middle & 0.221 & 0.019 & 0.183 & 0.258 & 0.086 & 2.148 & 986 & 0.138 & 0.015 & 0.108 & 0.167 & 0.109 & 1.926 & 1,164 \\
\hline Fourth & 0.241 & 0.019 & 0.204 & 0.278 & 0.078 & 2.212 & 1,081 & 0.129 & 0.013 & 0.103 & 0.155 & 0.103 & 1.626 & 1,192 \\
\hline Highest & 0.195 & 0.016 & 0.163 & 0.226 & 0.083 & 2.176 & 1,221 & 0.106 & 0.013 & 0.080 & 0.131 & 0.122 & 2.118 & 1,318 \\
\hline
\end{tabular}


Table S26 Indicator: Disability un-incidence

\begin{tabular}{|c|c|c|c|c|c|c|c|c|c|c|c|c|c|c|}
\hline \multirow[b]{3}{*}{ Characteristic } & \multirow[b]{3}{*}{ Estimate } & \multirow[b]{3}{*}{ SE } & \multicolumn{3}{|c|}{ Male } & \multirow{3}{*}{$\begin{array}{l}\text { Design } \\
\text { effect }\end{array}$} & \multirow[b]{3}{*}{ Count } & \multirow[b]{3}{*}{ Estimate } & \multirow[b]{3}{*}{ SE } & \multirow{2}{*}{\multicolumn{2}{|c|}{$\begin{array}{l}\text { Female } \\
\text { 95\% C.I }\end{array}$}} & \multirow{3}{*}{$\begin{array}{l}\text { CV } \\
(\%)\end{array}$} & \multirow{3}{*}{$\begin{array}{l}\text { Design } \\
\text { effect }\end{array}$} & \multirow[b]{3}{*}{ Count } \\
\hline & & & & C.I & CV & & & & & & & & & \\
\hline & & & Lower & Upper & (\%) & & & & & Lower & Upper & & & \\
\hline \multicolumn{15}{|l|}{ Urban-rural residence } \\
\hline Urban & 0.989 & 0.003 & 0.983 & 0.995 & 0.003 & 1.425 & 1,622 & 0.990 & 0.004 & 0.981 & 0.998 & 0.004 & 2.942 & 1,692 \\
\hline Rural & 0.981 & 0.003 & 0.975 & 0.988 & 0.003 & 1.931 & 2,957 & 0.989 & 0.003 & 0.984 & 0.994 & 0.003 & 1.955 & 3,546 \\
\hline Informal urban areas & 0.986 & 0.005 & 0.975 & 0.996 & 0.005 & 1.001 & 494 & 0.993 & 0.003 & 0.986 & 1.000 & 0.003 & 0.882 & 605 \\
\hline \multicolumn{15}{|l|}{ Region } \\
\hline Urban Governorates & 0.987 & 0.004 & 0.980 & 0.994 & 0.004 & 1.124 & 1,025 & 0.993 & 0.003 & 0.988 & 0.999 & 0.003 & 1.259 & 1,039 \\
\hline Urban Lower Egypt & 0.994 & 0.003 & 0.988 & 0.999 & 0.003 & 0.786 & 576 & 0.993 & 0.004 & 0.985 & 1.001 & 0.004 & 1.490 & 641 \\
\hline Rural Lower Egypt & 0.982 & 0.004 & 0.974 & 0.989 & 0.004 & 1.576 & 1,618 & 0.990 & 0.003 & 0.985 & 0.996 & 0.003 & 1.289 & 1,808 \\
\hline Urban Upper Egypt & 0.984 & 0.008 & 0.967 & 1.000 & 0.008 & 1.935 & 289 & 0.979 & 0.014 & 0.952 & 1.006 & 0.014 & 3.987 & 350 \\
\hline Rural Upper Egypt & 0.981 & 0.005 & 0.970 & 0.991 & 0.005 & 2.338 & 1,110 & 0.987 & 0.004 & 0.978 & 0.996 & 0.005 & 2.456 & 1,509 \\
\hline Frontier Governorates & 0.993 & 0.003 & 0.987 & 1.000 & 0.003 & 0.174 & 455 & 1.000 & 0.000 & 0.000 & 0.000 & 0.000 & 0.000 & 496 \\
\hline \multicolumn{15}{|l|}{ Age group } \\
\hline $13-17$ years & 0.986 & 0.005 & 0.977 & 0.996 & 0.005 & 1.892 & 1,091 & 0.994 & 0.003 & 0.988 & 0.999 & 0.003 & 1.327 & 1,105 \\
\hline $18-24$ years & 0.990 & 0.002 & 0.986 & 0.995 & 0.002 & 1.237 & 2,080 & 0.989 & 0.004 & 0.982 & 0.997 & 0.004 & 2.527 & 2,078 \\
\hline $25-29$ years & 0.981 & 0.005 & 0.972 & 0.990 & 0.005 & 1.536 & 1,108 & 0.989 & 0.005 & 0.979 & 1.000 & 0.005 & 3.136 & 1,456 \\
\hline $30-35$ years & 0.970 & 0.008 & 0.953 & 0.986 & 0.009 & 2.086 & 794 & 0.985 & 0.004 & 0.977 & 0.993 & 0.004 & 1.084 & 1,204 \\
\hline \multicolumn{15}{|l|}{ Education level } \\
\hline Illiterate & 0.930 & 0.022 & 0.887 & 0.973 & 0.024 & 2.369 & 288 & 0.976 & 0.007 & 0.963 & 0.990 & 0.007 & 1.443 & 831 \\
\hline Read and write & 0.988 & 0.012 & 0.964 & 1.012 & 0.012 & 0.491 & 38 & 0.989 & 0.011 & 0.968 & 1.010 & 0.011 & 0.739 & 83 \\
\hline Primary & 0.967 & 0.009 & 0.949 & 0.985 & 0.010 & 1.323 & 455 & 0.973 & 0.014 & 0.945 & 1.000 & 0.014 & 2.789 & 439 \\
\hline Preparatory & 0.992 & 0.003 & 0.986 & 0.999 & 0.003 & 1.104 & 646 & 0.994 & 0.002 & 0.989 & 0.999 & 0.002 & 0.843 & 872 \\
\hline General secondary & 0.982 & 0.008 & 0.967 & 0.998 & 0.008 & 1.580 & 417 & 0.997 & 0.003 & 0.992 & 1.003 & 0.003 & 1.263 & 516 \\
\hline Vocational secondary & 0.989 & 0.003 & 0.983 & 0.995 & 0.003 & 1.865 & 2,027 & 0.992 & 0.003 & 0.986 & 0.998 & 0.003 & 2.037 & 1,952 \\
\hline Post-secondary institute & 1.000 & 0.000 & 0.000 & 0.000 & 0.000 & 0.000 & 188 & 0.984 & 0.016 & 0.951 & 1.016 & 0.017 & 2.498 & 165 \\
\hline University and above & 0.990 & 0.003 & 0.984 & 0.997 & 0.003 & 1.221 & 1,014 & 0.994 & 0.003 & 0.987 & 1.000 & 0.003 & 1.794 & 982 \\
\hline \multicolumn{15}{|l|}{ Wealth quintile } \\
\hline Lowest & 0.983 & 0.004 & 0.975 & 0.992 & 0.004 & 1.104 & 829 & 0.990 & 0.005 & 0.981 & 0.999 & 0.005 & 2.267 & 1,021 \\
\hline Second & 0.976 & 0.007 & 0.962 & 0.990 & 0.007 & 2.442 & 954 & 0.987 & 0.006 & 0.976 & 0.998 & 0.006 & 2.779 & 1,145 \\
\hline Middle & 0.983 & 0.005 & 0.973 & 0.993 & 0.005 & 1.602 & 987 & 0.991 & 0.004 & 0.984 & 0.998 & 0.004 & 1.373 & 1,165 \\
\hline Fourth & 0.985 & 0.005 & 0.976 & 0.994 & 0.005 & 1.616 & 1,082 & 0.990 & 0.003 & 0.984 & 0.997 & 0.003 & 1.234 & 1,192 \\
\hline Highest & 0.992 & 0.003 & 0.987 & 0.997 & 0.003 & 1.127 & 1,221 & 0.989 & 0.004 & 0.982 & 0.996 & 0.004 & 1.517 & 1,320 \\
\hline
\end{tabular}


Table S27 Indicator: Youth free of chronic health conditions (diabetes, heart problems, respiratory, and kidney-related diseases)

\begin{tabular}{|c|c|c|c|c|c|c|c|c|c|c|c|c|c|c|}
\hline \multirow[b]{3}{*}{ Characteristic } & \multirow[b]{3}{*}{ Estimate } & \multirow[b]{3}{*}{ SE } & \multicolumn{3}{|c|}{ Male } & \multirow{3}{*}{$\begin{array}{l}\text { Design } \\
\text { effect }\end{array}$} & \multirow[b]{3}{*}{ Count } & \multirow[b]{3}{*}{ Estimate } & \multirow[b]{3}{*}{ SE } & \multirow{2}{*}{\multicolumn{2}{|c|}{$\begin{array}{l}\text { Female } \\
95 \% \text { C.I }\end{array}$}} & \multirow{3}{*}{$\begin{array}{l}\text { CV } \\
\text { (\%) }\end{array}$} & \multirow{3}{*}{$\begin{array}{l}\text { Design } \\
\text { effect }\end{array}$} & \multirow[b]{3}{*}{ Count } \\
\hline & & & \multicolumn{2}{|c|}{ 95\% C.I } & \multirow{2}{*}{$\begin{array}{l}\mathrm{CV} \\
(\%)\end{array}$} & & & & & & & & & \\
\hline & & & Lower & Upper & & & & & & Lower & Upper & & & \\
\hline Urban-rural residence & & & & & & & & & & & & & & \\
\hline Urban & 0.844 & 0.017 & 0.811 & 0.877 & 0.020 & 3.620 & 1,622 & 0.714 & 0.019 & 0.677 & 0.752 & 0.027 & 2.736 & 1,692 \\
\hline Rural & 0.778 & 0.016 & 0.747 & 0.809 & 0.020 & 4.893 & 2,957 & 0.728 & 0.014 & 0.700 & 0.756 & 0.020 & 3.345 & 3,546 \\
\hline Informal urban areas & 0.764 & 0.036 & 0.693 & 0.836 & 0.048 & 3.920 & 494 & 0.608 & 0.034 & 0.542 & 0.674 & 0.055 & 2.554 & 605 \\
\hline Region & & & & & & & & & & & & & & \\
\hline Urban Governorates & 0.868 & 0.016 & 0.836 & 0.900 & 0.019 & 2.508 & 1,025 & 0.711 & 0.020 & 0.672 & 0.750 & 0.028 & 1.855 & 1,039 \\
\hline Rural Lower Egypt & 0.807 & 0.022 & 0.763 & 0.851 & 0.028 & 5.762 & 1,618 & 0.725 & 0.022 & 0.682 & 0.769 & 0.031 & 4.054 & 1,808 \\
\hline Urban Upper Egypt & 0.738 & 0.043 & 0.652 & 0.823 & 0.059 & 4.416 & 289 & 0.681 & 0.035 & 0.613 & 0.749 & 0.051 & 2.346 & 350 \\
\hline Rural Upper Egypt & 0.738 & 0.022 & 0.695 & 0.781 & 0.030 & 3.831 & 1,110 & 0.726 & 0.018 & 0.690 & 0.761 & 0.025 & 2.626 & 1,509 \\
\hline Frontier Governorates & 0.897 & 0.027 & 0.844 & 0.950 & 0.030 & 0.799 & 455 & 0.872 & 0.027 & 0.819 & 0.925 & 0.031 & 0.636 & 496 \\
\hline Age group & & & & & & & & & & & & & & \\
\hline 13-17 years & 0.805 & 0.017 & 0.771 & 0.839 & 0.021 & 2.093 & 1,091 & 0.738 & 0.017 & 0.705 & 0.772 & 0.023 & 1.699 & 1,105 \\
\hline $18-24$ years & 0.803 & 0.014 & 0.776 & 0.830 & 0.017 & 2.818 & 2,080 & 0.718 & 0.014 & 0.691 & 0.745 & 0.019 & 1.844 & 2,078 \\
\hline $25-29$ years & 0.793 & 0.017 & 0.760 & 0.826 & 0.021 & 2.246 & 1,108 & 0.700 & 0.017 & 0.667 & 0.733 & 0.024 & 1.683 & 1,456 \\
\hline $30-35$ years & 0.772 & 0.020 & 0.732 & 0.812 & 0.026 & 2.041 & 794 & 0.686 & 0.018 & 0.651 & 0.721 & 0.026 & 1.434 & 1,204 \\
\hline Illiterate & 0.751 & 0.036 & 0.680 & 0.822 & 0.048 & 2.219 & 288 & 0.713 & 0.022 & 0.671 & 0.756 & 0.030 & 1.512 & 831 \\
\hline Read and write & 0.786 & 0.078 & 0.633 & 0.939 & 0.099 & 1.415 & 38 & 0.622 & 0.058 & 0.508 & 0.737 & 0.094 & 0.977 & 83 \\
\hline Primary & 0.760 & 0.027 & 0.707 & 0.812 & 0.035 & 1.910 & 455 & 0.675 & 0.029 & 0.618 & 0.732 & 0.043 & 1.475 & 439 \\
\hline Preparatory & 0.778 & 0.022 & 0.734 & 0.821 & 0.029 & 2.024 & 646 & 0.701 & 0.020 & 0.662 & 0.740 & 0.028 & 1.524 & 872 \\
\hline General secondary & 0.781 & 0.027 & 0.728 & 0.834 & 0.034 & 1.847 & 417 & 0.761 & 0.022 & 0.717 & 0.804 & 0.029 & 1.317 & 516 \\
\hline Vocational secondary & 0.805 & 0.013 & 0.779 & 0.831 & 0.017 & 2.591 & 2,027 & 0.700 & 0.015 & 0.671 & 0.730 & 0.021 & 1.886 & 1,952 \\
\hline Post-secondary institute & 0.744 & 0.043 & 0.659 & 0.828 & 0.058 & 1.992 & 188 & 0.736 & 0.038 & 0.662 & 0.811 & 0.052 & 1.132 & 165 \\
\hline University and above & 0.835 & 0.016 & 0.803 & 0.867 & 0.019 & 2.212 & 1,014 & 0.736 & 0.018 & 0.701 & 0.770 & 0.024 & 1.571 & 982 \\
\hline Wealth quintile & & & & & & & & & & & & & & \\
\hline Lowest & 0.775 & 0.019 & 0.738 & 0.812 & 0.024 & 2.016 & 829 & 0.704 & 0.021 & 0.663 & 0.745 & 0.030 & 2.105 & 1,021 \\
\hline Second & 0.784 & 0.022 & 0.741 & 0.827 & 0.028 & 3.143 & 954 & 0.675 & 0.019 & 0.638 & 0.712 & 0.028 & 1.745 & 1,145 \\
\hline Middle & 0.789 & 0.021 & 0.747 & 0.831 & 0.027 & 2.795 & 987 & 0.691 & 0.019 & 0.654 & 0.729 & 0.028 & 1.756 & 1,165 \\
\hline Fourth & 0.822 & 0.017 & 0.789 & 0.854 & 0.020 & 2.160 & 1,082 & 0.743 & 0.017 & 0.708 & 0.777 & 0.023 & 1.641 & 1,192 \\
\hline Highest & 0.807 & 0.017 & 0.773 & 0.840 & 0.021 & 2.499 & 1,221 & 0.744 & 0.017 & 0.710 & 0.778 & 0.023 & 1.914 & 1,320 \\
\hline
\end{tabular}




\begin{tabular}{|c|c|c|c|c|c|c|c|c|c|c|c|c|c|c|}
\hline \multirow[b]{3}{*}{ Characteristic } & \multirow[b]{3}{*}{ Estimate } & \multirow[b]{3}{*}{ SE } & \multirow{2}{*}{\multicolumn{2}{|c|}{$\begin{aligned} & \text { Male } \\
& 95 \% \text { C.I }\end{aligned}$}} & \multirow{3}{*}{$\begin{array}{l}\text { CV } \\
\text { (\%) }\end{array}$} & \multirow{3}{*}{$\begin{array}{c}\text { Design } \\
\text { effect }\end{array}$} & \multirow[b]{3}{*}{ Count } & \multirow[b]{3}{*}{ Estimate } & \multirow[b]{3}{*}{ SE } & \multirow{2}{*}{\multicolumn{2}{|c|}{$\begin{array}{l}\text { Female } \\
95 \% \text { C.I }\end{array}$}} & \multirow{3}{*}{$\begin{array}{l}\text { CV } \\
\text { (\%) }\end{array}$} & \multirow{3}{*}{$\begin{array}{c}\text { Design } \\
\text { effect }\end{array}$} & \multirow[b]{3}{*}{ Count } \\
\hline & & & & & & & & & & & & & & \\
\hline & & & Lower & Upper & & & & & & Lower & Upper & & & \\
\hline \multicolumn{15}{|l|}{ Urban-rural residence } \\
\hline Urban & 0.349 & 0.015 & 0.321 & 0.378 & 0.042 & 1.563 & 1,622 & 0.032 & 0.005 & 0.022 & 0.043 & 0.162 & 1.351 & 1,692 \\
\hline Rural & 0.317 & 0.012 & 0.292 & 0.341 & 0.039 & 2.406 & 2,957 & 0.026 & 0.004 & 0.019 & 0.034 & 0.148 & 1.925 & 3,546 \\
\hline Informal urban areas & 0.330 & 0.038 & 0.255 & 0.405 & 0.116 & 3.508 & 494 & 0.037 & 0.011 & 0.016 & 0.057 & 0.286 & 1.677 & 605 \\
\hline \multicolumn{15}{|l|}{ Region } \\
\hline Urban Governorates & 0.378 & 0.016 & 0.346 & 0.409 & 0.043 & 1.190 & 1,025 & 0.033 & 0.007 & 0.019 & 0.048 & 0.218 & 1.587 & 1,039 \\
\hline Urban Lower Egypt & 0.260 & 0.024 & 0.213 & 0.306 & 0.091 & 1.837 & 576 & 0.038 & 0.009 & 0.020 & 0.055 & 0.235 & 1.358 & 641 \\
\hline Rural Lower Egypt & 0.262 & 0.014 & 0.234 & 0.290 & 0.054 & 1.847 & 1,618 & 0.021 & 0.004 & 0.014 & 0.028 & 0.176 & 1.065 & 1,808 \\
\hline Urban Upper Egypt & 0.399 & 0.039 & 0.322 & 0.476 & 0.098 & 2.907 & 289 & 0.028 & 0.010 & 0.009 & 0.046 & 0.343 & 1.429 & 350 \\
\hline Rural Upper Egypt & 0.381 & 0.019 & 0.344 & 0.418 & 0.049 & 2.316 & 1,110 & 0.031 & 0.007 & 0.017 & 0.045 & 0.224 & 2.556 & 1,509 \\
\hline Frontier Governorates & 0.254 & 0.030 & 0.194 & 0.313 & 0.119 & 0.489 & 455 & 0.051 & 0.013 & 0.026 & 0.076 & 0.250 & 0.325 & 496 \\
\hline \multicolumn{15}{|l|}{ Age group } \\
\hline $13-17$ years & 0.105 & 0.011 & 0.084 & 0.126 & 0.103 & 1.369 & 1,091 & 0.034 & 0.007 & 0.021 & 0.047 & 0.200 & 1.578 & 1,105 \\
\hline $18-24$ years & 0.300 & 0.013 & 0.275 & 0.325 & 0.043 & 1.842 & 2,080 & 0.030 & 0.005 & 0.020 & 0.039 & 0.161 & 1.580 & 2,078 \\
\hline $25-29$ years & 0.445 & 0.020 & 0.407 & 0.484 & 0.044 & 2.027 & 1,108 & 0.023 & 0.005 & 0.014 & 0.032 & 0.200 & 1.179 & 1,456 \\
\hline $30-35$ years & 0.510 & 0.022 & 0.466 & 0.554 & 0.044 & 1.752 & 794 & 0.030 & 0.006 & 0.019 & 0.041 & 0.183 & 1.017 & 1,204 \\
\hline \multicolumn{15}{|l|}{ Education level } \\
\hline Illiterate & 0.424 & 0.035 & 0.355 & 0.492 & 0.083 & 1.606 & 288 & 0.027 & 0.007 & 0.014 & 0.041 & 0.249 & 1.167 & 831 \\
\hline Read and write & 0.493 & 0.096 & 0.304 & 0.682 & 0.195 & 1.455 & 38 & 0.013 & 0.010 & -0.007 & 0.033 & 0.787 & 0.552 & 83 \\
\hline Primary & 0.455 & 0.029 & 0.398 & 0.512 & 0.063 & 1.649 & 455 & 0.022 & 0.009 & 0.005 & 0.039 & 0.390 & 1.322 & 439 \\
\hline Preparatory & 0.324 & 0.022 & 0.280 & 0.368 & 0.069 & 1.591 & 646 & 0.031 & 0.007 & 0.017 & 0.045 & 0.232 & 1.387 & 872 \\
\hline General secondary & 0.155 & 0.020 & 0.115 & 0.194 & 0.130 & 1.360 & 417 & 0.036 & 0.010 & 0.015 & 0.056 & 0.289 & 1.506 & 516 \\
\hline Vocational secondary & 0.364 & 0.015 & 0.334 & 0.395 & 0.042 & 2.315 & 2,027 & 0.030 & 0.004 & 0.021 & 0.038 & 0.150 & 1.219 & 1,952 \\
\hline Post-secondary institute & 0.346 & 0.041 & 0.265 & 0.428 & 0.120 & 1.553 & 188 & 0.015 & 0.009 & -0.003 & 0.034 & 0.600 & 0.861 & 165 \\
\hline University and above & 0.234 & 0.016 & 0.202 & 0.267 & 0.070 & 1.703 & 1,014 & 0.031 & 0.007 & 0.018 & 0.044 & 0.211 & 1.417 & 982 \\
\hline \multicolumn{15}{|l|}{ Wealth quintile } \\
\hline Lowest & 0.404 & 0.021 & 0.363 & 0.445 & 0.051 & 1.776 & 829 & 0.018 & 0.004 & 0.010 & 0.025 & 0.214 & 0.843 & 1,021 \\
\hline Second & 0.365 & 0.021 & 0.324 & 0.406 & 0.057 & 2.096 & 954 & 0.045 & 0.008 & 0.029 & 0.062 & 0.186 & 1.773 & 1,145 \\
\hline Middle & 0.322 & 0.018 & 0.288 & 0.357 & 0.054 & 1.436 & 987 & 0.026 & 0.006 & 0.015 & 0.037 & 0.211 & 1.202 & 1,165 \\
\hline Fourth & 0.305 & 0.017 & 0.272 & 0.339 & 0.056 & 1.572 & 1,082 & 0.027 & 0.005 & 0.017 & 0.037 & 0.186 & 0.988 & 1,192 \\
\hline Highest & 0.263 & 0.016 & 0.231 & 0.294 & 0.062 & 1.789 & 1,221 & 0.029 & 0.006 & 0.017 & 0.040 & 0.209 & 1.548 & 1,320 \\
\hline
\end{tabular}


Table S29 Indicator: Percentage of youth having tried drugs

\begin{tabular}{|c|c|c|c|c|c|c|c|c|c|c|c|c|c|c|}
\hline \multirow[b]{3}{*}{ Characteristic } & \multirow[b]{3}{*}{ Estimate } & \multirow[b]{3}{*}{ SE } & \multicolumn{3}{|c|}{ Male } & \multirow{3}{*}{$\begin{array}{c}\text { Design } \\
\text { effect }\end{array}$} & \multirow[b]{3}{*}{ Count } & \multirow[b]{3}{*}{ Estimate } & \multirow[b]{3}{*}{ SE } & \multirow{2}{*}{\multicolumn{2}{|c|}{$\begin{array}{l}\text { Female } \\
95 \% \text { C.I }\end{array}$}} & \multirow{3}{*}{$\begin{array}{l}\text { CV } \\
(\%)\end{array}$} & \multirow{3}{*}{$\begin{array}{c}\text { Design } \\
\text { effect }\end{array}$} & \multirow[b]{3}{*}{ Count } \\
\hline & & & 95 & C.I & CV & & & & & & & & & \\
\hline & & & Lower & Upper & (\%) & & & & & Lower & Upper & & & \\
\hline \multicolumn{15}{|l|}{ Urban-rural residence } \\
\hline Urban & 0.024 & 0.004 & 0.017 & 0.032 & 0.160 & 1.071 & 1,622 & 0.002 & 0.001 & 0.000 & 0.004 & 0.498 & 0.770 & 1,692 \\
\hline Rural & 0.016 & 0.004 & 0.009 & 0.023 & 0.229 & 2.892 & 2,957 & 0.000 & 0.000 & 0.000 & 0.001 & 0.706 & 0.604 & 3,546 \\
\hline Informal urban areas & 0.020 & 0.007 & 0.006 & 0.035 & 0.367 & 1.474 & 494 & 0.000 & 0.000 & 0.000 & 0.000 & 0.000 & 0.000 & 605 \\
\hline \multicolumn{15}{|l|}{ Region } \\
\hline Urban Governorates & 0.030 & 0.005 & 0.020 & 0.041 & 0.180 & 1.095 & 1,025 & 0.003 & 0.002 & 0.000 & 0.006 & 0.492 & 0.754 & 1,039 \\
\hline Urban Lower Egypt & 0.015 & 0.005 & 0.005 & 0.025 & 0.338 & 1.097 & 576 & 0.000 & 0.000 & 0.000 & 0.000 & 0.000 & 0.000 & 641 \\
\hline Rural Lower Egypt & 0.012 & 0.003 & 0.006 & 0.019 & 0.274 & 1.675 & 1,618 & 0.001 & 0.001 & 0.000 & 0.002 & 0.704 & 0.600 & 1,808 \\
\hline Urban Upper Egypt & 0.020 & 0.008 & 0.005 & 0.035 & 0.378 & 1.327 & 289 & 0.000 & 0.000 & 0.000 & 0.000 & 0.000 & 0.000 & 350 \\
\hline Rural Upper Egypt & 0.021 & 0.007 & 0.007 & 0.034 & 0.335 & 3.651 & 1,110 & 0.000 & 0.000 & 0.000 & 0.000 & 0.000 & 0.000 & 1,509 \\
\hline Frontier Governorates & 0.005 & 0.004 & -0.003 & 0.013 & 0.760 & 0.310 & 455 & 0.000 & 0.000 & 0.000 & 0.000 & 0.000 & 0.000 & 496 \\
\hline \multicolumn{15}{|l|}{ Age group } \\
\hline $13-17$ years & 0.004 & 0.002 & 0.000 & 0.008 & 0.522 & 1.222 & 1,091 & 0.000 & 0.000 & 0.000 & 0.000 & 0.000 & 0.000 & 1,105 \\
\hline $18-24$ years & 0.018 & 0.003 & 0.012 & 0.025 & 0.185 & 1.452 & 2,080 & 0.001 & 0.001 & 0.000 & 0.003 & 0.507 & 0.746 & 2,078 \\
\hline $25-29$ years & 0.031 & 0.008 & 0.016 & 0.046 & 0.245 & 2.481 & 1,108 & 0.000 & 0.000 & 0.000 & 0.001 & 0.999 & 0.618 & 1,456 \\
\hline $30-35$ years & 0.022 & 0.006 & 0.010 & 0.034 & 0.275 & 1.503 & 794 & 0.001 & 0.001 & -0.001 & 0.002 & 1.000 & 0.794 & 1,204 \\
\hline \multicolumn{15}{|l|}{ Education level } \\
\hline Illiterate & 0.040 & 0.013 & 0.013 & 0.066 & 0.338 & 1.503 & 288 & 0.000 & 0.000 & 0.000 & 0.000 & 0.000 & 0.000 & 831 \\
\hline Read and write & 0.068 & 0.049 & 0.049 & 0.165 & 0.720 & 1.498 & 38 & 0.000 & 0.000 & 0.000 & 0.000 & 0.000 & 0.000 & 83 \\
\hline Primary & 0.022 & 0.006 & 0.006 & 0.034 & 0.268 & 0.807 & 455 & 0.000 & 0.000 & 0.000 & 0.000 & 0.000 & 0.000 & 439 \\
\hline Preparatory & 0.029 & 0.007 & 0.007 & 0.042 & 0.240 & 1.193 & 646 & 0.001 & 0.001 & -0.001 & 0.002 & 1.000 & 0.619 & 872 \\
\hline General secondary & 0.006 & 0.003 & 0.003 & 0.013 & 0.581 & 0.870 & 417 & 0.000 & 0.000 & 0.000 & 0.000 & 0.000 & 0.000 & 516 \\
\hline Vocational secondary & 0.015 & 0.004 & 0.004 & 0.022 & 0.252 & 2.172 & 2,027 & 0.001 & 0.000 & 0.000 & 0.002 & 0.707 & 0.605 & 1,952 \\
\hline Post-secondary institute & 0.058 & 0.033 & 0.033 & 0.122 & 0.567 & 4.024 & 188 & 0.000 & 0.000 & 0.000 & 0.000 & 0.000 & 0.000 & 165 \\
\hline University and above & 0.010 & 0.004 & 0.004 & 0.017 & 0.365 & 1.545 & 1,014 & 0.003 & 0.001 & 0.000 & 0.005 & 0.572 & 0.816 & 982 \\
\hline \multicolumn{15}{|l|}{ Wealth quintile } \\
\hline Lowest & 0.022 & 0.007 & 0.007 & 0.036 & 0.338 & 2.503 & 829 & 0.000 & 0.000 & 0.000 & 0.000 & 0.000 & 0.000 & 1,021 \\
\hline Second & 0.025 & 0.006 & 0.013 & 0.038 & 0.253 & 1.870 & 954 & 0.000 & 0.000 & 0.000 & 0.000 & 0.000 & 0.000 & 1,145 \\
\hline Middle & 0.021 & 0.005 & 0.011 & 0.032 & 0.254 & 1.428 & 987 & 0.001 & 0.001 & -0.001 & 0.002 & 0.999 & 0.617 & 1,165 \\
\hline Fourth & 0.012 & 0.004 & 0.005 & 0.019 & 0.309 & 1.271 & 1,082 & 0.000 & 0.000 & 0.000 & 0.000 & 0.000 & 0.000 & 1,192 \\
\hline Highest & 0.016 & 0.005 & 0.007 & 0.026 & 0.301 & 1.947 & 1,221 & 0.003 & 0.001 & 0.000 & 0.006 & 0.446 & 0.737 & 1,320 \\
\hline
\end{tabular}


Table S30 Indicator: Percentage of girls having undergone FGM/C

\begin{tabular}{|c|c|c|c|c|c|c|c|c|c|c|c|c|c|c|}
\hline \multirow[b]{3}{*}{ Characteristic } & \multirow[b]{3}{*}{ Estimate } & \multirow[b]{3}{*}{ SE } & \multicolumn{3}{|c|}{ Male } & \multirow{3}{*}{$\begin{array}{l}\text { Design } \\
\text { effect }\end{array}$} & \multirow[b]{3}{*}{ Count } & \multirow[b]{3}{*}{ Estimate } & \multirow[b]{3}{*}{ SE } & \multirow{2}{*}{\multicolumn{2}{|c|}{$\begin{array}{l}\text { Female } \\
95 \% \text { C.I }\end{array}$}} & \multirow{3}{*}{$\begin{array}{l}\text { CV } \\
\text { (\%) }\end{array}$} & \multirow{3}{*}{$\begin{array}{c}\text { Design } \\
\text { effect }\end{array}$} & \multirow[b]{3}{*}{ Count } \\
\hline & & & 95 & C.I & CV & & & & & & & & & \\
\hline & & & Lower & Upper & $(\%)$ & & & & & Lower & Upper & & & \\
\hline \multicolumn{15}{|l|}{ Urban-rural residence } \\
\hline Urban & & & & & & & & 0.643 & 0.025 & 0.594 & 0.692 & 0.039 & 4.602 & 1,692 \\
\hline Rural & & & & & & & & 0.869 & 0.010 & 0.849 & 0.889 & 0.012 & 3.320 & 3,546 \\
\hline Informal urban areas & & & & & & & & 0.727 & 0.037 & 0.655 & 0.799 & 0.050 & 3.964 & 605 \\
\hline \multicolumn{15}{|l|}{ Region } \\
\hline Urban Governorates & & & & & & & & 0.534 & 0.025 & 0.485 & 0.584 & 0.047 & 2.725 & 1,039 \\
\hline Urban Lower Egypt & & & & & & & & 0.723 & 0.031 & 0.662 & 0.785 & 0.043 & 3.362 & 641 \\
\hline Rural Lower Egypt & & & & & & & & 0.837 & 0.016 & 0.806 & 0.869 & 0.019 & 3.298 & 1,808 \\
\hline Urban Upper Egypt & & & & & & & & 0.876 & 0.035 & 0.806 & 0.945 & 0.040 & 5.349 & 350 \\
\hline Rural Upper Egypt & & & & & & & & 0.905 & 0.012 & 0.881 & 0.929 & 0.014 & 3.136 & 1,509 \\
\hline Frontier Governorates & & & & & & & & 0.698 & 0.055 & 0.590 & 0.807 & 0.079 & 1.528 & 496 \\
\hline \multicolumn{15}{|l|}{ Age group } \\
\hline $13-17$ years & & & & & & & & 0.718 & 0.017 & 0.684 & 0.753 & 0.024 & 1.862 & 1,105 \\
\hline $18-24$ years & & & & & & & & 0.781 & 0.016 & 0.750 & 0.812 & 0.020 & 3.119 & 2,078 \\
\hline $25-29$ years & & & & & & & & 0.821 & 0.015 & 0.792 & 0.850 & 0.018 & 1.976 & 1,456 \\
\hline $30-35$ years & & & & & & & & 0.850 & 0.014 & 0.822 & 0.877 & 0.016 & 1.585 & 1,204 \\
\hline \multicolumn{15}{|l|}{ Education level } \\
\hline Illiterate & & & & & & & & 0.893 & 0.016 & 0.863 & 0.924 & 0.017 & 1.857 & 831 \\
\hline Read and write & & & & & & & & 0.836 & 0.050 & 0.738 & 0.934 & 0.060 & 1.347 & 83 \\
\hline Primary & & & & & & & & 0.834 & 0.023 & 0.789 & 0.880 & 0.027 & 1.611 & 439 \\
\hline Preparatory & & & & & & & & 0.814 & 0.017 & 0.781 & 0.848 & 0.021 & 1.673 & 872 \\
\hline General secondary & & & & & & & & 0.637 & 0.028 & 0.581 & 0.692 & 0.045 & 1.841 & 516 \\
\hline Vocational secondary & & & & & & & & 0.827 & 0.013 & 0.802 & 0.853 & 0.016 & 2.276 & 1,952 \\
\hline Post-secondary institute & & & & & & & & 0.747 & 0.039 & 0.671 & 0.823 & 0.052 & 1.317 & 165 \\
\hline University and above & & & & & & & & 0.696 & 0.023 & 0.651 & 0.741 & 0.033 & 2.700 & 982 \\
\hline \multicolumn{15}{|l|}{ Wealth quintile } \\
\hline Lowest & & & & & & & & 0.858 & 0.014 & 0.831 & 0.885 & 0.016 & 1.757 & 1,021 \\
\hline Second & & & & & & & & 0.822 & 0.017 & 0.788 & 0.856 & 0.021 & 2.352 & 1,145 \\
\hline Middle & & & & & & & & 0.817 & 0.016 & 0.785 & 0.849 & 0.020 & 1.995 & 1,165 \\
\hline Fourth & & & & & & & & 0.772 & 0.018 & 0.737 & 0.807 & 0.023 & 2.032 & 1,192 \\
\hline Highest & & & & & & & & 0.697 & 0.022 & 0.653 & 0.740 & 0.032 & 3.055 & 1,320 \\
\hline
\end{tabular}


Table S31 Indicator: Percentage of married youth

\begin{tabular}{|c|c|c|c|c|c|c|c|c|c|c|c|c|c|c|}
\hline \multirow[b]{3}{*}{ Characteristic } & \multirow[b]{3}{*}{ Estimate } & \multirow[b]{3}{*}{ SE } & \multicolumn{3}{|c|}{ Male } & \multirow{3}{*}{$\begin{array}{c}\text { Design } \\
\text { effect }\end{array}$} & \multirow[b]{3}{*}{ Count } & \multirow[b]{3}{*}{ Estimate } & \multirow[b]{3}{*}{ SE } & \multicolumn{2}{|c|}{ Female } & \multirow{3}{*}{$\begin{array}{l}\text { CV } \\
(\%)\end{array}$} & \multirow{3}{*}{$\begin{array}{c}\text { Design } \\
\text { effect }\end{array}$} & \multirow[b]{3}{*}{ Count } \\
\hline & & & 95 & C.I & CV & & & & & & C.I & & & \\
\hline & & & Lower & Upper & (\%) & & & & & Lower & Upper & & & \\
\hline \multicolumn{15}{|l|}{ Urban-rural residence } \\
\hline Urban & 0.213 & 0.012 & 0.188 & 0.237 & 0.059 & 1.566 & 1,621 & 0.357 & 0.015 & 0.328 & 0.386 & 0.041 & 1.445 & 1,690 \\
\hline Rural & 0.256 & 0.010 & 0.235 & 0.276 & 0.040 & 1.893 & 2,954 & 0.520 & 0.011 & 0.498 & 0.543 & 0.022 & 1.661 & 3,544 \\
\hline Informal urban areas & 0.253 & 0.024 & 0.206 & 0.300 & 0.094 & 1.591 & 492 & 0.448 & 0.027 & 0.395 & 0.500 & 0.060 & 1.545 & 605 \\
\hline \multicolumn{15}{|l|}{ Region } \\
\hline Urban Governorates & 0.219 & 0.015 & 0.189 & 0.249 & 0.069 & 1.441 & 1,025 & 0.367 & 0.018 & 0.331 & 0.403 & 0.050 & 1.384 & 1,037 \\
\hline Urban Lower Egypt & 0.212 & 0.021 & 0.172 & 0.253 & 0.097 & 1.617 & 573 & 0.418 & 0.024 & 0.371 & 0.465 & 0.057 & 1.473 & 641 \\
\hline Rural Lower Egypt & 0.282 & 0.013 & 0.257 & 0.308 & 0.047 & 1.529 & 1,618 & 0.540 & 0.014 & 0.511 & 0.568 & 0.027 & 1.357 & 1,808 \\
\hline Urban Upper Egypt & 0.241 & 0.029 & 0.184 & 0.297 & 0.119 & 2.027 & 289 & 0.356 & 0.033 & 0.291 & 0.420 & 0.092 & 2.001 & 350 \\
\hline Rural Upper Egypt & 0.224 & 0.016 & 0.193 & 0.255 & 0.070 & 2.177 & 1,107 & 0.503 & 0.017 & 0.469 & 0.537 & 0.035 & 1.937 & 1,507 \\
\hline Frontier Governorates & 0.262 & 0.029 & 0.205 & 0.319 & 0.111 & 0.443 & 455 & 0.416 & 0.030 & 0.358 & 0.475 & 0.072 & 0.352 & 496 \\
\hline \multicolumn{15}{|l|}{ Age group } \\
\hline $13-17$ years & 0.000 & 0.000 & 0.000 & 0.000 & 0.000 & 0.000 & 1,091 & 0.034 & 0.006 & 0.023 & 0.045 & 0.169 & 1.127 & 1,105 \\
\hline $18-24$ years & 0.068 & 0.007 & 0.055 & 0.081 & 0.098 & 1.621 & 2,078 & 0.333 & 0.014 & 0.306 & 0.360 & 0.041 & 1.682 & 2,078 \\
\hline $25-29$ years & 0.418 & 0.019 & 0.380 & 0.455 & 0.046 & 1.948 & 1,106 & 0.749 & 0.016 & 0.718 & 0.780 & 0.021 & 1.624 & 1,453 \\
\hline $30-35$ years & 0.755 & 0.021 & 0.714 & 0.796 & 0.027 & 2.006 & 792 & 0.881 & 0.014 & 0.853 & 0.909 & 0.016 & 1.903 & 1,203 \\
\hline \multicolumn{15}{|l|}{ Education level } \\
\hline Illiterate & 0.354 & 0.033 & 0.289 & 0.419 & 0.094 & 1.528 & 287 & 0.723 & 0.022 & 1.682 & 0.000 & 0.031 & 1.682 & 831 \\
\hline Read and write & 0.361 & 0.083 & 0.198 & 0.523 & 0.229 & 1.166 & 38 & 0.800 & 0.062 & 1.625 & 0.000 & 0.078 & 1.625 & 83 \\
\hline Primary & 0.345 & 0.023 & 0.299 & 0.391 & 0.068 & 1.184 & 454 & 0.559 & 0.028 & 1.247 & 0.000 & 0.051 & 1.247 & 437 \\
\hline Preparatory & 0.167 & 0.016 & 0.135 & 0.199 & 0.097 & 1.316 & 645 & 0.370 & 0.019 & 1.197 & 0.000 & 0.050 & 1.197 & 871 \\
\hline General secondary & 0.073 & 0.016 & 0.042 & 0.103 & 0.214 & 1.573 & 417 & 0.144 & 0.018 & 1.255 & 0.000 & 0.124 & 1.255 & 516 \\
\hline Vocational secondary & 0.260 & 0.012 & 0.237 & 0.284 & 0.046 & 1.701 & 2,027 & 0.553 & 0.015 & 1.665 & 0.000 & 0.028 & 1.665 & 1,952 \\
\hline Post-secondary institute & 0.282 & 0.039 & 0.205 & 0.359 & 0.139 & 1.550 & 188 & 0.460 & 0.045 & 1.255 & 0.000 & 0.098 & 1.255 & 165 \\
\hline University and above & 0.232 & 0.017 & 0.199 & 0.264 & 0.071 & 1.765 & 1,011 & 0.316 & 0.015 & 1.086 & 0.000 & 0.049 & 1.086 & 981 \\
\hline \multicolumn{15}{|l|}{ Wealth quintile } \\
\hline Lowest & 0.215 & 0.017 & 0.181 & 0.249 & 0.080 & 1.742 & 827 & 0.483 & 0.021 & 0.442 & 0.523 & 0.043 & 1.724 & 1,021 \\
\hline Second & 0.227 & 0.016 & 0.197 & 0.258 & 0.069 & 1.548 & 952 & 0.461 & 0.018 & 0.426 & 0.495 & 0.038 & 1.328 & 1,144 \\
\hline Middle & 0.270 & 0.018 & 0.235 & 0.305 & 0.066 & 1.633 & 987 & 0.482 & 0.016 & 0.450 & 0.514 & 0.034 & 1.070 & 1,164 \\
\hline Fourth & 0.251 & 0.015 & 0.221 & 0.280 & 0.060 & 1.357 & 1,081 & 0.467 & 0.018 & 0.433 & 0.502 & 0.038 & 1.295 & 1,192 \\
\hline Highest & 0.247 & 0.014 & 0.219 & 0.275 & 0.058 & 1.435 & 1,220 & 0.443 & 0.018 & 0.407 & 0.478 & 0.041 & 1.591 & 1,318 \\
\hline
\end{tabular}


Table S32 Indicator: Percentage of married youth who live with their parents (extended families)

\begin{tabular}{|c|c|c|c|c|c|c|c|c|c|c|c|c|c|c|}
\hline \multirow[b]{3}{*}{ Characteristic } & \multirow[b]{3}{*}{ Estimate } & \multirow[b]{3}{*}{ SE } & \multirow{2}{*}{\multicolumn{2}{|c|}{$\begin{array}{r}\text { Male } \\
95 \% \text { C.I }\end{array}$}} & \multirow{3}{*}{$\begin{array}{l}\text { CV } \\
(\%)\end{array}$} & \multirow{3}{*}{$\begin{array}{c}\text { Design } \\
\text { effect }\end{array}$} & \multirow[b]{3}{*}{ Count } & \multirow[b]{3}{*}{ Estimate } & \multirow[b]{3}{*}{ SE } & \multirow{2}{*}{\multicolumn{2}{|c|}{$\begin{array}{l}\text { Female } \\
95 \% \text { C.I }\end{array}$}} & \multirow{3}{*}{$\begin{array}{l}\text { CV } \\
(\%)\end{array}$} & \multirow{3}{*}{$\begin{array}{l}\text { Design } \\
\text { effect }\end{array}$} & \multirow[b]{3}{*}{ Count } \\
\hline & & & & & & & & & & & & & & \\
\hline & & & Lower & Upper & & & & & & Lower & Upper & & & \\
\hline \multicolumn{15}{|l|}{ Urban-rural residence } \\
\hline Urban & 0.401 & 0.034 & 0.334 & 0.468 & 0.085 & 2.085 & 389 & 0.248 & 0.024 & 0.202 & 0.294 & 0.095 & 1.943 & 769 \\
\hline Rural & 0.484 & 0.024 & 0.438 & 0.531 & 0.049 & 2.311 & 848 & 0.426 & 0.018 & 0.391 & 0.460 & 0.041 & 2.544 & 2,146 \\
\hline Informal urban areas & 0.276 & 0.068 & 0.142 & 0.410 & 0.247 & 3.750 & 129 & 0.107 & 0.041 & 0.026 & 0.189 & 0.386 & 5.170 & 316 \\
\hline \multicolumn{15}{|l|}{ Region } \\
\hline Urban Governorates & 0.325 & 0.037 & 0.375 & 0.251 & 0.115 & 1.811 & 247 & 0.213 & 0.023 & 0.226 & 0.169 & 0.106 & 1.297 & 470 \\
\hline Urban Lower Egypt & 0.253 & 0.059 & 0.592 & 0.136 & 0.234 & 2.997 & 138 & 0.133 & 0.039 & 0.394 & 0.056 & 0.296 & 4.201 & 325 \\
\hline Rural Lower Egypt & 0.414 & 0.027 & 0.273 & 0.361 & 0.066 & 1.858 & 503 & 0.326 & 0.021 & 0.210 & 0.285 & 0.064 & 2.107 & 1,125 \\
\hline Urban Upper Egypt & 0.592 & 0.064 & 0.635 & 0.468 & 0.107 & 2.180 & 72 & 0.318 & 0.063 & 0.631 & 0.194 & 0.199 & 3.332 & 158 \\
\hline Rural Upper Egypt & 0.585 & 0.040 & 0.402 & 0.506 & 0.069 & 2.727 & 270 & 0.536 & 0.025 & 0.247 & 0.487 & 0.046 & 2.342 & 900 \\
\hline Frontier Governorates & 0.488 & 0.077 & 0.770 & 0.336 & 0.158 & 0.762 & 136 & 0.264 & 0.057 & 0.567 & 0.152 & 0.215 & 0.796 & 253 \\
\hline \multicolumn{15}{|l|}{ Age group } \\
\hline $13-17$ years & 0.000 & 0.000 & 0.000 & 0.000 & 0.000 & 0.000 & 44 & 0.352 & 0.074 & 0.206 & 0.498 & 0.211 & 1.104 & 0.000 \\
\hline $18-24$ years & 0.447 & 0.046 & 0.356 & 0.538 & 0.103 & 1.633 & 156 & 0.342 & 0.021 & 0.300 & 0.384 & 0.062 & 1.598 & 874 \\
\hline $25-29$ years & 0.473 & 0.028 & 0.419 & 0.528 & 0.059 & 2.028 & 558 & 0.363 & 0.019 & 0.326 & 0.401 & 0.052 & 1.730 & 1,194 \\
\hline $30-35$ years & 0.414 & 0.024 & 0.366 & 0.462 & 0.059 & 1.937 & 649 & 0.358 & 0.020 & 0.318 & 0.397 & 0.056 & 1.775 & 1,119 \\
\hline \multicolumn{15}{|l|}{ Education level } \\
\hline Illiterate & 0.354 & 0.033 & 0.289 & 0.419 & 0.094 & 1.528 & 122 & 0.723 & 0.022 & 0.678 & 0.767 & 0.031 & 1.682 & 659 \\
\hline Read and write & 0.361 & 0.083 & 0.198 & 0.523 & 0.229 & 1.166 & 18 & 0.800 & 0.062 & 0.678 & 0.922 & 0.078 & 1.625 & 73 \\
\hline Primary & 0.345 & 0.023 & 0.299 & 0.391 & 0.068 & 1.184 & 178 & 0.559 & 0.028 & 0.503 & 0.615 & 0.051 & 1.247 & 277 \\
\hline Preparatory & 0.167 & 0.016 & 0.135 & 0.199 & 0.097 & 1.316 & 120 & 0.370 & 0.019 & 0.334 & 0.407 & 0.050 & 1.197 & 385 \\
\hline General secondary & 0.073 & 0.016 & 0.042 & 0.103 & 0.214 & 1.573 & 27 & 0.144 & 0.018 & 0.109 & 0.180 & 0.124 & 1.255 & 98 \\
\hline Vocational secondary & 0.260 & 0.012 & 0.237 & 0.284 & 0.046 & 1.701 & 599 & 0.553 & 0.015 & 0.523 & 0.583 & 0.028 & 1.665 & 1,249 \\
\hline Post-secondary institute & 0.282 & 0.039 & 0.205 & 0.359 & 0.139 & 1.550 & 56 & 0.460 & 0.045 & 0.371 & 0.549 & 0.098 & 1.255 & 91 \\
\hline University and above & 0.232 & 0.017 & 0.199 & 0.264 & 0.071 & 1.765 & 243 & 0.316 & 0.015 & 0.286 & 0.347 & 0.049 & 1.086 & 397 \\
\hline \multicolumn{15}{|l|}{ Wealth quintile } \\
\hline Lowest & 0.588 & 0.036 & 0.517 & 0.660 & 0.062 & 1.392 & 216 & 0.522 & 0.027 & 0.470 & 0.575 & 0.051 & 1.658 & 589 \\
\hline Second & 0.559 & 0.039 & 0.483 & 0.635 & 0.069 & 1.835 & 242 & 0.415 & 0.026 & 0.365 & 0.465 & 0.062 & 1.596 & 638 \\
\hline Middle & 0.438 & 0.037 & 0.366 & 0.511 & 0.084 & 1.826 & 279 & 0.344 & 0.026 & 0.292 & 0.396 & 0.077 & 1.801 & 648 \\
\hline Fourth & 0.363 & 0.036 & 0.293 & 0.433 & 0.098 & 1.866 & 299 & 0.310 & 0.024 & 0.263 & 0.358 & 0.078 & 1.561 & 656 \\
\hline Highest & 0.326 & 0.032 & 0.262 & 0.389 & 0.099 & 1.861 & 330 & 0.201 & 0.019 & 0.164 & 0.237 & 0.093 & 1.390 & 700 \\
\hline
\end{tabular}


Table S33 Indicator: Ideal number of children

\begin{tabular}{|c|c|c|c|c|c|c|c|c|c|c|c|c|c|c|}
\hline \multirow[b]{3}{*}{ Characteristic } & \multirow[b]{3}{*}{ Estimate } & \multirow[b]{3}{*}{ SE } & \multicolumn{3}{|c|}{ Male } & \multirow{3}{*}{$\begin{array}{c}\text { Design } \\
\text { effect }\end{array}$} & \multirow[b]{3}{*}{ Count } & \multirow[b]{3}{*}{ Estimate } & \multirow[b]{3}{*}{ SE } & \multirow{2}{*}{\multicolumn{2}{|c|}{$\begin{array}{l}\text { Female } \\
95 \% \text { C.I }\end{array}$}} & \multirow{3}{*}{$\begin{array}{l}\text { CV } \\
\text { (\%) }\end{array}$} & \multirow{3}{*}{$\begin{array}{c}\text { Design } \\
\text { effect }\end{array}$} & \multirow[b]{3}{*}{ Count } \\
\hline & & & 95 & C.I & CV & & & & & & & & & \\
\hline & & & Lower & Upper & (\%) & & & & & Lower & Upper & & & \\
\hline \multicolumn{15}{|l|}{ Urban-rural residence } \\
\hline Urban & 2.798 & 0.049 & 2.701 & 2.895 & 0.018 & 4.006 & 1,621 & 2.773 & 0.049 & 2.677 & 2.868 & 0.018 & 3.883 & 1,690 \\
\hline Rural & 3.124 & 0.041 & 3.044 & 3.205 & 0.013 & 4.573 & 2,953 & 3.141 & 0.036 & 3.070 & 3.212 & 0.011 & 3.637 & 3,544 \\
\hline Informal urban areas & 2.961 & 0.077 & 2.809 & 3.113 & 0.026 & 3.450 & 493 & 3.040 & 0.079 & 2.884 & 3.196 & 0.026 & 3.131 & 605 \\
\hline \multicolumn{15}{|l|}{ Region } \\
\hline Urban Governorates & 2.693 & 0.042 & 2.610 & 2.776 & 0.016 & 1.826 & 1,025 & 2.625 & 0.039 & 2.549 & 2.702 & 0.015 & 1.797 & 1,037 \\
\hline Urban Lower Egypt & 2.740 & 0.063 & 2.617 & 2.863 & 0.023 & 3.145 & 574 & 2.820 & 0.049 & 2.724 & 2.915 & 0.017 & 1.739 & 641 \\
\hline Rural Lower Egypt & 2.832 & 0.037 & 2.759 & 2.904 & 0.013 & 2.612 & 1,617 & 2.844 & 0.036 & 2.774 & 2.915 & 0.013 & 2.132 & 1,808 \\
\hline Urban Upper Egypt & 3.325 & 0.093 & 3.142 & 3.508 & 0.028 & 4.417 & 289 & 3.381 & 0.100 & 3.184 & 3.578 & 0.030 & 3.669 & 350 \\
\hline Rural Upper Egypt & 3.459 & 0.059 & 3.342 & 3.575 & 0.017 & 3.987 & 1,107 & 3.442 & 0.049 & 3.345 & 3.539 & 0.014 & 3.332 & 1,507 \\
\hline Frontier Governorates & 3.029 & 0.105 & 2.822 & 3.236 & 0.035 & 0.971 & 455 & 2.952 & 0.111 & 2.732 & 3.171 & 0.038 & 0.874 & 496 \\
\hline \multicolumn{15}{|l|}{ Age group } \\
\hline $13-17$ years & 2.943 & 0.046 & 2.853 & 3.033 & 0.016 & 2.093 & 1,090 & 2.836 & 0.038 & 2.761 & 2.910 & 0.013 & 1.633 & 1,105 \\
\hline $18-24$ years & 2.989 & 0.039 & 2.913 & 3.065 & 0.013 & 3.068 & 2,078 & 2.952 & 0.038 & 2.877 & 3.028 & 0.013 & 2.576 & 2,078 \\
\hline $25-29$ years & 3.018 & 0.046 & 2.928 & 3.109 & 0.015 & 2.295 & 1,106 & 3.137 & 0.039 & 3.061 & 3.214 & 0.012 & 1.825 & 1,453 \\
\hline $30-35$ years & 3.141 & 0.051 & 3.041 & 3.241 & 0.016 & 1.864 & 793 & 3.249 & 0.043 & 3.164 & 3.335 & 0.013 & 1.520 & 1,203 \\
\hline \multicolumn{15}{|l|}{ Education level } \\
\hline Illiterate & 3.250 & 0.087 & 3.078 & 3.422 & 0.027 & 1.856 & 287 & 3.478 & 0.054 & 3.371 & 3.585 & 0.016 & 1.483 & 831 \\
\hline Read and write & 2.803 & 0.140 & 2.527 & 3.079 & 0.050 & 1.123 & 38 & 3.315 & 0.118 & 3.082 & 3.547 & 0.036 & 0.874 & 83 \\
\hline Primary & 3.075 & 0.064 & 2.950 & 3.201 & 0.021 & 1.305 & 453 & 3.209 & 0.066 & 3.079 & 3.339 & 0.021 & 1.302 & 437 \\
\hline Preparatory & 3.019 & 0.055 & 2.910 & 3.127 & 0.018 & 1.933 & 645 & 3.082 & 0.048 & 2.988 & 3.176 & 0.016 & 1.633 & 871 \\
\hline General secondary & 2.808 & 0.054 & 2.702 & 2.914 & 0.019 & 1.341 & 417 & 2.720 & 0.055 & 2.613 & 2.828 & 0.020 & 1.579 & 516 \\
\hline Vocational secondary & 3.109 & 0.044 & 3.023 & 3.194 & 0.014 & 3.617 & 2,027 & 3.017 & 0.035 & 2.949 & 3.086 & 0.012 & 2.028 & 1,952 \\
\hline Post-secondary institute & 2.906 & 0.088 & 2.733 & 3.078 & 0.030 & 1.702 & 188 & 2.901 & 0.083 & 2.738 & 3.064 & 0.029 & 1.154 & 165 \\
\hline University and above & 2.821 & 0.046 & 2.730 & 2.911 & 0.016 & 2.501 & 1,012 & 2.764 & 0.040 & 2.686 & 2.843 & 0.014 & 1.889 & 981 \\
\hline \multicolumn{15}{|l|}{ Wealth quintile } \\
\hline Lowest & 3.269 & 0.060 & 3.151 & 3.387 & 0.018 & 2.108 & 827 & 3.301 & 0.052 & 3.198 & 3.404 & 0.016 & 2.091 & 1,021 \\
\hline Second & 3.122 & 0.058 & 3.008 & 3.236 & 0.019 & 3.096 & 951 & 3.171 & 0.045 & 3.083 & 3.258 & 0.014 & 1.889 & 1,144 \\
\hline Middle & 2.996 & 0.045 & 2.908 & 3.084 & 0.015 & 2.068 & 987 & 3.009 & 0.044 & 2.924 & 3.095 & 0.014 & 1.798 & 1,164 \\
\hline Fourth & 2.979 & 0.041 & 2.898 & 3.059 & 0.014 & 1.905 & 1,081 & 2.875 & 0.036 & 2.803 & 2.946 & 0.013 & 1.530 & 1,192 \\
\hline Highest & 2.760 & 0.038 & 2.685 & 2.835 & 0.014 & 2.298 & 1,221 & 2.806 & 0.039 & 2.730 & 2.883 & 0.014 & 1.800 & 1,318 \\
\hline
\end{tabular}


Table S34 Indicator: Ideal number of children for married people

\begin{tabular}{|c|c|c|c|c|c|c|c|c|c|c|c|c|c|c|}
\hline \multirow[b]{3}{*}{ Characteristic } & \multirow[b]{3}{*}{ Estimate } & \multirow[b]{3}{*}{ SE } & \multicolumn{3}{|c|}{ Male } & \multirow{3}{*}{$\begin{array}{l}\text { Design } \\
\text { effect }\end{array}$} & \multirow[b]{3}{*}{ Count } & \multirow[b]{3}{*}{ Estimate } & \multirow[b]{3}{*}{ SE } & \multicolumn{2}{|c|}{ Female } & \multirow{3}{*}{$\begin{array}{l}\text { CV } \\
(\%)\end{array}$} & \multirow{3}{*}{$\begin{array}{c}\text { Design } \\
\text { effect }\end{array}$} & \multirow[b]{3}{*}{ Count } \\
\hline & & & \multicolumn{2}{|c|}{ 95\% C.I } & \multirow{2}{*}{$\begin{array}{l}\text { CV } \\
(\%)\end{array}$} & & & & & 95 & C.I & & & \\
\hline & & & Lower & Upper & & & & & & Lower & Upper & & & \\
\hline \multicolumn{15}{|l|}{ Urban-rural residence } \\
\hline Urban & 2.970 & 0.089 & 2.795 & 3.145 & 0.030 & 1.911 & 389 & 2.926 & 0.059 & 2.811 & 3.042 & 0.020 & 2.294 & 769 \\
\hline Rural & 3.236 & 0.054 & 3.131 & 3.342 & 0.017 & 2.448 & 843 & 3.279 & 0.041 & 3.198 & 3.361 & 0.013 & 2.781 & 2,147 \\
\hline Informal urban areas & 3.088 & 0.113 & 2.866 & 3.310 & 0.037 & 2.443 & 130 & 3.153 & 0.097 & 2.963 & 3.343 & 0.031 & 2.473 & 316 \\
\hline \multicolumn{15}{|l|}{ Region } \\
\hline Urban Governorates & 2.790 & 0.096 & 2.600 & 2.979 & 0.035 & 1.265 & 247 & 2.717 & 0.052 & 2.616 & 2.819 & 0.019 & 1.367 & 470 \\
\hline Urban Lower Egypt & 2.893 & 0.088 & 2.721 & 3.065 & 0.030 & 1.558 & 139 & 2.995 & 0.063 & 2.872 & 3.119 & 0.021 & 1.575 & 325 \\
\hline Rural Lower Egypt & 2.962 & 0.055 & 2.854 & 3.071 & 0.019 & 1.950 & 501 & 2.981 & 0.045 & 2.893 & 3.069 & 0.015 & 1.885 & 1,126 \\
\hline Urban Upper Egypt & 3.585 & 0.151 & 3.289 & 3.882 & 0.042 & 3.478 & 72 & 3.661 & 0.111 & 3.444 & 3.878 & 0.030 & 1.736 & 158 \\
\hline Rural Upper Egypt & 3.628 & 0.086 & 3.458 & 3.797 & 0.024 & 2.247 & 268 & 3.603 & 0.058 & 3.488 & 3.717 & 0.016 & 2.732 & 900 \\
\hline Frontier Governorates & 3.320 & 0.165 & 2.996 & 3.645 & 0.050 & 0.757 & 135 & 3.149 & 0.137 & 2.880 & 3.418 & 0.043 & 0.524 & 253 \\
\hline \multicolumn{15}{|l|}{ Age group } \\
\hline $13-17$ years & 0.000 & 0.000 & 0.000 & 0.000 & 0.000 & 0.000 & 44 & 3.165 & 0.214 & 2.744 & 3.586 & 0.068 & 1.419 & 0.000 \\
\hline $18-24$ years & 3.030 & 0.079 & 2.875 & 3.186 & 0.026 & 1.320 & 156 & 3.014 & 0.050 & 2.916 & 3.111 & 0.016 & 1.489 & 875 \\
\hline $25-29$ years & 3.138 & 0.062 & 3.017 & 3.260 & 0.020 & 1.650 & 557 & 3.221 & 0.041 & 3.140 & 3.301 & 0.013 & 1.747 & 1,194 \\
\hline $30-35$ years & 3.191 & 0.058 & 3.076 & 3.305 & 0.018 & 2.023 & 649 & 3.292 & 0.044 & 3.206 & 3.378 & 0.013 & 1.604 & 1,119 \\
\hline \multicolumn{15}{|l|}{ Education level } \\
\hline Illiterate & 3.250 & 0.087 & 3.078 & 3.422 & 0.027 & 1.856 & 121 & 3.478 & 0.054 & 3.371 & 3.585 & 0.016 & 1.483 & 659 \\
\hline Read and write & 2.803 & 0.140 & 2.527 & 3.079 & 0.050 & 1.123 & 18 & 3.315 & 0.118 & 3.082 & 3.547 & 0.036 & 0.874 & 73 \\
\hline Primary & 3.075 & 0.064 & 2.950 & 3.201 & 0.021 & 1.305 & 178 & 3.209 & 0.066 & 3.079 & 3.339 & 0.021 & 1.302 & 277 \\
\hline Preparatory & 3.019 & 0.055 & 2.910 & 3.127 & 0.018 & 1.933 & 120 & 3.082 & 0.048 & 2.988 & 3.176 & 0.016 & 1.633 & 385 \\
\hline General secondary & 2.808 & 0.054 & 2.702 & 2.914 & 0.019 & 1.341 & 27 & 2.720 & 0.055 & 2.613 & 2.828 & 0.020 & 1.579 & 98 \\
\hline Vocational secondary & 3.109 & 0.044 & 3.023 & 3.194 & 0.014 & 3.617 & 599 & 3.017 & 0.035 & 2.949 & 3.086 & 0.012 & 2.028 & 1,250 \\
\hline Post-secondary institute & 2.906 & 0.088 & 2.733 & 3.078 & 0.030 & 1.702 & 56 & 2.901 & 0.083 & 2.738 & 3.064 & 0.029 & 1.154 & 91 \\
\hline University and above & 2.821 & 0.046 & 2.730 & 2.911 & 0.016 & 2.501 & 243 & 2.764 & 0.040 & 2.686 & 2.843 & 0.014 & 1.889 & 397 \\
\hline \multicolumn{15}{|l|}{ Wealth quintile } \\
\hline Lowest & 3.565 & 0.124 & 3.322 & 3.808 & 0.035 & 1.448 & 213 & 3.469 & 0.064 & 3.343 & 3.594 & 0.018 & 1.705 & 589 \\
\hline Second & 3.208 & 0.098 & 3.016 & 3.400 & 0.031 & 2.036 & 241 & 3.314 & 0.054 & 3.208 & 3.420 & 0.016 & 1.418 & 638 \\
\hline Middle & 3.175 & 0.070 & 3.038 & 3.312 & 0.022 & 1.992 & 279 & 3.179 & 0.054 & 3.073 & 3.285 & 0.017 & 1.579 & 649 \\
\hline Fourth & 3.129 & 0.074 & 2.983 & 3.275 & 0.024 & 1.692 & 299 & 3.087 & 0.045 & 3.000 & 3.175 & 0.014 & 1.288 & 656 \\
\hline Highest & 2.836 & 0.066 & 2.707 & 2.966 & 0.023 & 2.017 & 330 & 2.921 & 0.054 & 2.815 & 3.027 & 0.019 & 1.484 & 700 \\
\hline
\end{tabular}


Table S35 Indicator: Ideal number of children for unmarried people

\begin{tabular}{|c|c|c|c|c|c|c|c|c|c|c|c|c|c|c|}
\hline \multirow[b]{3}{*}{ Characteristic } & \multirow[b]{3}{*}{ Estimate } & \multirow[b]{3}{*}{ SE } & \multirow{2}{*}{\multicolumn{2}{|c|}{$\begin{array}{r}\text { Male } \\
\text { 95\% C.I }\end{array}$}} & \multirow{3}{*}{$\begin{array}{l}\mathrm{CV} \\
(\%)\end{array}$} & \multirow{3}{*}{$\begin{array}{l}\text { Design } \\
\text { effect }\end{array}$} & \multirow[b]{3}{*}{ Count } & \multirow[b]{3}{*}{ Estimate } & \multirow[b]{3}{*}{ SE } & \multirow{2}{*}{\multicolumn{2}{|c|}{$\begin{array}{l}\text { Female } \\
95 \% \text { C.l }\end{array}$}} & \multirow{3}{*}{$\begin{array}{l}\text { CV } \\
(\%)\end{array}$} & \multirow{3}{*}{$\begin{array}{l}\text { Design } \\
\text { effect }\end{array}$} & \multirow[b]{3}{*}{ Count } \\
\hline & & & & & & & & & & & & & & \\
\hline & & & Lower & Upper & & & & & & Lower & Upper & & & \\
\hline \multicolumn{15}{|l|}{ Urban-rural residence } \\
\hline Urban & 2.737 & 0.124 & 2.493 & 2.980 & 0.045 & 1.522 & 134 & 2.794 & 0.086 & 2.625 & 2.963 & 0.031 & 1.221 & 249 \\
\hline Rural & 3.262 & 0.124 & 3.018 & 3.507 & 0.038 & 1.782 & 193 & 3.324 & 0.088 & 3.151 & 3.497 & 0.026 & 1.846 & 430 \\
\hline Informal urban areas & 2.880 & 0.130 & 2.625 & 3.136 & 0.045 & 1.208 & 30 & 3.136 & 0.115 & 2.910 & 3.362 & 0.037 & 0.706 & 59 \\
\hline \multicolumn{15}{|l|}{ Region } \\
\hline Urban Governorates & 2.635 & 0.165 & 2.309 & 2.960 & 0.063 & 1.626 & 91 & 2.698 & 0.091 & 2.520 & 2.877 & 0.034 & 0.872 & 152 \\
\hline Urban Lower Egypt & 2.660 & 0.142 & 2.380 & 2.940 & 0.054 & 1.494 & 38 & 3.014 & 0.101 & 2.820 & 3.212 & 0.033 & 0.941 & 87 \\
\hline Rural Lower Egypt & 2.995 & 0.111 & 2.778 & 3.213 & 0.037 & 1.122 & 116 & 3.111 & 0.105 & 2.900 & 3.318 & 0.034 & 1.685 & 235 \\
\hline Urban Upper Egypt & 3.262 & 0.134 & 2.998 & 3.526 & 0.041 & 0.910 & 20 & 3.056 & 0.333 & 2.400 & 3.710 & 0.109 & 2.296 & 31 \\
\hline Rural Upper Egypt & 3.677 & 0.267 & 3.151 & 4.202 & 0.073 & 2.392 & 53 & 3.569 & 0.144 & 3.290 & 3.853 & 0.040 & 1.989 & 166 \\
\hline Frontier Governorates & 3.384 & 0.194 & 3.002 & 3.766 & 0.057 & 0.393 & 39 & 3.307 & 0.171 & 2.970 & 3.644 & 0.052 & 0.333 & 67 \\
\hline \multicolumn{15}{|l|}{ Age group } \\
\hline $13-17$ years & 0.000 & 0.000 & 0.000 & 0.000 & 0.000 & 0.000 & 18 & 3.247 & 0.398 & 2.460 & 4.030 & 0.123 & 1.914 & 0.000 \\
\hline 18-24 years & 2.930 & 0.121 & 2.692 & 3.168 & 0.041 & 1.084 & 43 & 3.032 & 0.123 & 2.790 & 3.274 & 0.041 & 1.605 & 235 \\
\hline $25-29$ years & 3.008 & 0.117 & 2.778 & 3.239 & 0.039 & 1.791 & 146 & 3.205 & 0.074 & 3.060 & 3.351 & 0.023 & 1.150 & 275 \\
\hline $30-35$ years & 3.100 & 0.142 & 2.822 & 3.379 & 0.046 & 1.654 & 168 & 3.166 & 0.091 & 2.990 & 3.346 & 0.029 & 1.076 & 210 \\
\hline \multicolumn{15}{|l|}{ Education level } \\
\hline Illiterate & 3.407 & 0.129 & 3.155 & 3.660 & 0.038 & 1.850 & 16 & 3.576 & 0.057 & 3.460 & 3.689 & 0.016 & 1.442 & 78 \\
\hline Read and write & 3.110 & 0.127 & 2.860 & 3.360 & 0.041 & 0.876 & 5 & 3.330 & 0.118 & 3.100 & 3.563 & 0.036 & 0.834 & 12 \\
\hline Primary & 3.257 & 0.128 & 3.005 & 3.510 & 0.039 & 1.296 & 41 & 3.237 & 0.075 & 3.090 & 3.386 & 0.023 & 1.222 & 70 \\
\hline Preparatory & 3.228 & 0.122 & 2.988 & 3.469 & 0.038 & 1.691 & 26 & 3.199 & 0.065 & 3.070 & 3.328 & 0.020 & 1.362 & 79 \\
\hline General secondary & 2.713 & 0.195 & 2.329 & 3.097 & 0.072 & 1.590 & 8 & 3.305 & 0.153 & 3.000 & 3.606 & 0.046 & 1.403 & 27 \\
\hline Vocational secondary & 3.193 & 0.059 & 3.078 & 3.308 & 0.018 & 2.131 & 154 & 3.098 & 0.039 & 3.020 & 3.176 & 0.013 & 1.601 & 293 \\
\hline Post-secondary institute & 2.960 & 0.126 & 2.713 & 3.207 & 0.043 & 1.500 & 17 & 3.135 & 0.118 & 2.900 & 3.366 & 0.037 & 1.212 & 24 \\
\hline University and above & 2.945 & 0.092 & 2.765 & 3.125 & 0.031 & 2.133 & 90 & 2.805 & 0.057 & 2.690 & 2.918 & 0.020 & 1.354 & 155 \\
\hline \multicolumn{15}{|l|}{ Wealth quintile } \\
\hline Lowest & 3.469 & 0.415 & 2.653 & 4.286 & 0.120 & 1.571 & 34 & 3.433 & 0.154 & 3.130 & 3.737 & 0.045 & 1.468 & 89 \\
\hline Second & 3.113 & 0.236 & 2.649 & 3.576 & 0.076 & 1.901 & 50 & 3.221 & 0.102 & 3.020 & 3.421 & 0.032 & 0.948 & 134 \\
\hline Middle & 2.947 & 0.131 & 2.689 & 3.205 & 0.044 & 1.696 & 80 & 3.125 & 0.132 & 2.870 & 3.384 & 0.042 & 1.532 & 151 \\
\hline Fourth & 3.260 & 0.194 & 2.879 & 3.642 & 0.059 & 1.740 & 83 & 3.160 & 0.122 & 2.920 & 3.400 & 0.038 & 1.153 & 165 \\
\hline Highest & 2.768 & 0.135 & 2.502 & 3.034 & 0.049 & 2.299 & 110 & 2.918 & 0.096 & 2.730 & 3.107 & 0.033 & 1.083 & 199 \\
\hline
\end{tabular}


Table S36 Indicator: Average age at marriage for males

\begin{tabular}{|c|c|c|c|c|c|c|c|}
\hline \multirow[b]{3}{*}{ Characteristic } & \multirow[b]{3}{*}{ Estimate } & \multirow[b]{3}{*}{ SE } & \multicolumn{3}{|c|}{ Male } & \multirow{3}{*}{$\begin{array}{c}\text { Design } \\
\text { effect }\end{array}$} & \\
\hline & & & \multicolumn{2}{|c|}{ 95\% C.I } & \multirow{2}{*}{$\begin{array}{l}\text { CV } \\
(\%)\end{array}$} & & \\
\hline & & & Lower & Upper & & & \\
\hline \multicolumn{7}{|l|}{ Urban-rural residence } & Count \\
\hline Urban & 25.367 & 0.197 & 24.979 & 25.755 & 0.008 & 1.577 & 390 \\
\hline Rural & 24.222 & 0.138 & 23.951 & 24.493 & 0.006 & 1.837 & 845 \\
\hline Informal urban areas & 24.439 & 0.307 & 23.836 & 25.042 & 0.013 & 1.422 & 129 \\
\hline \multicolumn{8}{|l|}{ Region } \\
\hline Urban Governorates & 25.442 & 0.223 & 25.005 & 25.880 & 0.009 & 1.506 & 247 \\
\hline Urban Lower Egypt & 25.026 & 0.297 & 24.441 & 25.610 & 0.012 & 1.182 & 138 \\
\hline Rural Lower Egypt & 24.394 & 0.159 & 24.081 & 24.706 & 0.007 & 1.576 & 501 \\
\hline Urban Upper Egypt & 24.729 & 0.446 & 23.852 & 25.607 & 0.018 & 2.552 & 72 \\
\hline Rural Upper Egypt & 24.039 & 0.248 & 23.553 & 24.526 & 0.010 & 2.264 & 268 \\
\hline Frontier Governorates & 22.950 & 0.678 & 21.617 & 24.283 & 0.030 & 0.575 & 138 \\
\hline \multicolumn{8}{|l|}{ Age group } \\
\hline 13-17 years & 0.000 & 0.000 & 0.000 & 0.000 & 0.000 & 0.000 & 44 \\
\hline $18-24$ years & 21.420 & 0.148 & 21.129 & 21.712 & 0.007 & 1.415 & 156 \\
\hline $25-29$ years & 24.337 & 0.108 & 24.124 & 24.550 & 0.004 & 1.390 & 558 \\
\hline $30-35$ years & 25.478 & 0.172 & 25.140 & 25.815 & 0.007 & 1.644 & 650 \\
\hline \multicolumn{8}{|l|}{ Education level } \\
\hline Illiterate & 23.695 & 0.350 & 23.010 & 24.380 & 0.015 & 1.518 & 121 \\
\hline Read and write & 22.517 & 0.992 & 20.570 & 24.470 & 0.044 & 1.255 & 18 \\
\hline Primary & 23.898 & 0.278 & 23.350 & 24.440 & 0.012 & 1.517 & 178 \\
\hline Preparatory & 22.826 & 0.350 & 22.140 & 23.510 & 0.015 & 1.596 & 120 \\
\hline General secondary & 24.547 & 0.593 & 23.380 & 25.710 & 0.024 & 1.992 & 27 \\
\hline Vocational secondary & 24.454 & 0.151 & 24.160 & 24.750 & 0.006 & 1.502 & 602 \\
\hline Post-secondary institute & 24.821 & 0.477 & 23.880 & 25.760 & 0.019 & 1.804 & 56 \\
\hline University and above & 26.337 & 0.208 & 25.930 & 26.750 & 0.008 & 1.838 & 242 \\
\hline \multicolumn{8}{|l|}{ Wealth quintile } \\
\hline Lowest & 23.680 & 0.255 & 23.179 & 24.180 & 0.011 & 1.532 & 213 \\
\hline Second & 24.487 & 0.276 & 23.945 & 25.030 & 0.011 & 2.024 & 242 \\
\hline Middle & 24.337 & 0.241 & 23.863 & 24.811 & 0.010 & 1.650 & 280 \\
\hline Fourth & 24.619 & 0.220 & 24.186 & 25.051 & 0.009 & 1.569 & 300 \\
\hline Highest & 25.274 & 0.188 & 24.905 & 25.642 & 0.007 & 1.504 & 329 \\
\hline
\end{tabular}


Table S37 Indicator: Average age at marriage for females

\begin{tabular}{|c|c|c|c|c|c|c|c|}
\hline \multirow[b]{3}{*}{ Characteristic } & \multirow[b]{3}{*}{ Estimate } & \multirow[b]{3}{*}{ SE } & \multicolumn{3}{|c|}{ Female } & \multirow{3}{*}{$\begin{array}{l}\text { Design } \\
\text { effect }\end{array}$} & \multirow[b]{3}{*}{ Count } \\
\hline & & & 95 & C.I & CV & & \\
\hline & & & Lower & Upper & (\%) & & \\
\hline \multicolumn{8}{|l|}{ Urban-rural residence } \\
\hline Urban & 21.154 & 0.168 & 20.823 & 21.485 & 0.008 & 1.660 & 771 \\
\hline Rural & 19.526 & 0.089 & 19.350 & 19.701 & 0.005 & 1.689 & 2,150 \\
\hline Informal urban areas & 20.673 & 0.284 & 20.116 & 21.231 & 0.014 & 1.896 & 316 \\
\hline \multicolumn{8}{|l|}{ Region } \\
\hline Urban Governorates & 21.178 & 0.231 & 20.700 & 21.630 & 0.011 & 1.958 & 470 \\
\hline Urban Lower Egypt & 21.001 & 0.254 & 20.500 & 21.500 & 0.012 & 1.665 & 325 \\
\hline Rural Lower Egypt & 19.842 & 0.100 & 19.600 & 20.040 & 0.005 & 1.206 & 1,126 \\
\hline Urban Upper Egypt & 20.687 & 0.289 & 20.100 & 21.260 & 0.014 & 1.603 & 158 \\
\hline Rural Upper Egypt & 19.174 & 0.145 & 18.900 & 19.460 & 0.008 & 2.028 & 900 \\
\hline Frontier Governorates & 20.147 & 0.410 & 19.300 & 20.950 & 0.020 & 0.404 & 258 \\
\hline \multicolumn{8}{|l|}{ Age group } \\
\hline $13-17$ years & 15.879 & 0.150 & 15.600 & 16.170 & 0.009 & 1.206 & 0.000 \\
\hline $18-24$ years & 18.949 & 0.087 & 18.800 & 19.120 & 0.005 & 1.369 & 879 \\
\hline $25-29$ years & 20.305 & 0.118 & 20.100 & 20.540 & 0.006 & 1.468 & 1,195 \\
\hline $30-35$ years & 20.659 & 0.135 & 20.400 & 20.920 & 0.007 & 1.329 & 1,119 \\
\hline \multicolumn{8}{|l|}{ Education level } \\
\hline Illiterate & 18.910 & 0.163 & 18.588 & 19.231 & 0.009 & 1.838 & 661 \\
\hline Read and write & 19.358 & 0.421 & 18.531 & 20.185 & 0.022 & 1.368 & 73 \\
\hline Primary & 19.099 & 0.214 & 18.679 & 19.519 & 0.011 & 0.832 & 278 \\
\hline Preparatory & 18.747 & 0.160 & 18.433 & 19.061 & 0.009 & 1.172 & 385 \\
\hline General secondary & 19.916 & 0.371 & 19.186 & 20.646 & 0.019 & 1.094 & 98 \\
\hline Vocational secondary & 20.135 & 0.096 & 19.947 & 20.323 & 0.005 & 1.249 & 1,251 \\
\hline Post-secondary institute & 21.681 & 0.254 & 21.181 & 22.181 & 0.012 & 1.339 & 91 \\
\hline University and above & 22.782 & 0.179 & 22.429 & 23.135 & 0.008 & 0.775 & 398 \\
\hline \multicolumn{8}{|l|}{ Wealth quintile } \\
\hline Lowest & 18.894 & 0.148 & 18.600 & 19.180 & 0.008 & 1.469 & 589 \\
\hline Second & 19.922 & 0.150 & 19.600 & 20.220 & 0.008 & 1.163 & 640 \\
\hline Middle & 19.970 & 0.154 & 19.700 & 20.270 & 0.008 & 1.292 & 650 \\
\hline Fourth & 19.981 & 0.135 & 19.700 & 20.250 & 0.007 & 1.264 & 656 \\
\hline Highest & 21.101 & 0.153 & 20.800 & 21.400 & 0.007 & 1.285 & 702 \\
\hline
\end{tabular}




\section{Table S38 Indicator: Percentage of females married before 18}

\begin{tabular}{|c|c|c|c|c|c|c|c|c|c|c|c|c|c|c|}
\hline \multirow[b]{3}{*}{ Characteristic } & \multirow[b]{3}{*}{ Estimate } & \multirow[b]{3}{*}{ SE } & \multicolumn{3}{|c|}{ Male } & \multirow{3}{*}{$\begin{array}{l}\text { Design } \\
\text { effect }\end{array}$} & \multirow[b]{3}{*}{ Count } & \multirow[b]{3}{*}{ Estimate } & \multirow[b]{3}{*}{ SE } & \multirow{2}{*}{\multicolumn{2}{|c|}{$\begin{array}{l}\text { Female } \\
5 \% \text { C.I }\end{array}$}} & \multirow{3}{*}{$\begin{array}{l}\text { CV } \\
(\%)\end{array}$} & \multirow{3}{*}{$\begin{array}{c}\text { Design } \\
\text { effect }\end{array}$} & \multirow[b]{3}{*}{ Count } \\
\hline & & & \multicolumn{2}{|c|}{ 95\% C.I } & \multirow{2}{*}{$\begin{array}{l}\text { CV } \\
(\%) \\
\end{array}$} & & & & & & & & & \\
\hline & & & Lower & Upper & & & & & & Lower & Upper & & & \\
\hline Urban-rural residence & & & & & & & & & & & & & & \\
\hline Urban & & & & & & & & 0.119 & 0.014 & 0.092 & 0.147 & 0.117 & 1.319 & 771 \\
\hline Rural & & & & & & & & 0.250 & 0.013 & 0.225 & 0.275 & 0.051 & 1.943 & 2,149 \\
\hline Informal urban areas & & & & & & & & 0.163 & 0.023 & 0.118 & 0.208 & 0.141 & 1.214 & 316 \\
\hline Region & & & & & & & & & & & & & & \\
\hline Urban Governorates & & & & & & & & 0.124 & 0.018 & 0.089 & 0.159 & 0.142 & 1.324 & 472 \\
\hline Urban Lower Egypt & & & & & & & & 0.133 & 0.023 & 0.088 & 0.179 & 0.172 & 1.553 & 325 \\
\hline Rural Lower Egypt & & & & & & & & 0.184 & 0.013 & 0.159 & 0.210 & 0.071 & 1.291 & 1,126 \\
\hline Urban Upper Egypt & & & & & & & & 0.154 & 0.025 & 0.104 & 0.204 & 0.166 & 0.982 & 158 \\
\hline Rural Upper Egypt & & & & & & & & 0.324 & 0.021 & 0.283 & 0.365 & 0.064 & 2.080 & 902 \\
\hline Frontier Governorates & & & & & & & & 0.140 & 0.027 & 0.087 & 0.194 & 0.194 & 0.320 & 253 \\
\hline Age group & & & & & & & & & & & & & & \\
\hline $10-14$ years & & & & & & & & 0.000 & 0.000 & 0.000 & 0.000 & 0.000 & 0.000 & 44 \\
\hline $15-17$ years & & & & & & & & 0.204 & 0.016 & 0.173 & 0.236 & 0.079 & 1.366 & 875 \\
\hline $18-24$ years & & & & & & & & 0.206 & 0.014 & 0.179 & 0.234 & 0.068 & 1.456 & 1,197 \\
\hline 25-29 years & & & & & & & & 0.191 & 0.013 & 0.165 & 0.217 & 0.069 & 1.253 & 1,120 \\
\hline Education level & & & & & & & & & & & & & & \\
\hline Illiterate & & & & & & & & 0.373 & 0.025 & 0.325 & 0.421 & 0.066 & 1.607 & 659 \\
\hline Read and write & & & & & & & & 0.380 & 0.059 & 0.265 & 0.495 & 0.154 & 1.023 & 73 \\
\hline Primary & & & & & & & & 0.321 & 0.030 & 0.262 & 0.380 & 0.094 & 1.186 & 279 \\
\hline Preparatory & & & & & & & & 0.382 & 0.027 & 0.329 & 0.435 & 0.070 & 1.201 & 386 \\
\hline General secondary & & & & & & & & 0.171 & 0.040 & 0.091 & 0.250 & 0.237 & 1.049 & 98 \\
\hline Vocational secondary & & & & & & & & 0.131 & 0.010 & 0.111 & 0.151 & 0.078 & 1.168 & 1,250 \\
\hline Post-secondary institute & & & & & & & & 0.016 & 0.013 & -0.010 & 0.042 & 0.808 & 0.986 & 91 \\
\hline University and above & & & & & & & & 0.012 & 0.005 & 0.002 & 0.022 & 0.439 & 0.957 & 398 \\
\hline Wealth quintile & & & & & & & & & & & & & & \\
\hline Lowest & & & & & & & & 0.356 & 0.021 & 0.315 & 0.397 & 0.058 & 1.187 & 589 \\
\hline Second & & & & & & & & 0.239 & 0.021 & 0.197 & 0.281 & 0.089 & 1.621 & 639 \\
\hline Middle & & & & & & & & 0.210 & 0.018 & 0.173 & 0.246 & 0.088 & 1.294 & 650 \\
\hline Fourth & & & & & & & & 0.167 & 0.017 & 0.132 & 0.201 & 0.105 & 1.368 & 656 \\
\hline Highest & & & & & & & & 0.104 & 0.012 & 0.081 & 0.127 & 0.113 & 1.043 & 702 \\
\hline
\end{tabular}


Table S39 Indicator: Proportion of youth playing any kind of sport

\begin{tabular}{|c|c|c|c|c|c|c|c|c|c|c|c|c|c|c|}
\hline \multirow[b]{3}{*}{ Characteristic } & \multirow[b]{3}{*}{ Estimate } & \multirow[b]{3}{*}{ SE } & \multicolumn{3}{|c|}{ Male } & \multirow{3}{*}{$\begin{array}{c}\text { Design } \\
\text { effect }\end{array}$} & \multirow[b]{3}{*}{ Count } & \multirow[b]{3}{*}{ Estimate } & \multirow[b]{3}{*}{ SE } & \multirow{2}{*}{\multicolumn{2}{|c|}{$\begin{array}{l}\text { Female } \\
95 \% \text { C.I }\end{array}$}} & \multirow{3}{*}{$\begin{array}{l}\text { CV } \\
\text { (\%) }\end{array}$} & \multirow{3}{*}{$\begin{array}{l}\text { Design } \\
\text { effect }\end{array}$} & \multirow[b]{3}{*}{ Count } \\
\hline & & & 95 & C.I & CV & & & & & & & & & \\
\hline & & & Lower & Upper & (\%) & & & & & Lower & Upper & & & \\
\hline \multicolumn{15}{|l|}{ Urban-rural residence } \\
\hline Urban & 0.646 & 0.027 & 0.594 & 0.700 & 0.042 & 5.349 & 1,622 & 0.448 & 0.021 & 0.407 & 0.490 & 0.047 & 2.731 & 1,692 \\
\hline Rural & 0.695 & 0.017 & 0.662 & 0.728 & 0.024 & 4.524 & 2,957 & 0.407 & 0.015 & 0.377 & 0.438 & 0.038 & 3.221 & 3,546 \\
\hline Informal urban areas & 0.565 & 0.045 & 0.477 & 0.654 & 0.080 & 4.420 & 494 & 0.287 & 0.043 & 0.203 & 0.372 & 0.150 & 4.851 & 605 \\
\hline \multicolumn{15}{|l|}{ Region } \\
\hline Urban Governorates & 0.666 & 0.026 & 0.616 & 0.716 & 0.038 & 3.146 & 1,025 & 0.439 & 0.025 & 0.390 & 0.488 & 0.056 & 2.398 & 1,039 \\
\hline Urban Lower Egypt & 0.551 & 0.044 & 0.465 & 0.637 & 0.079 & 4.905 & 576 & 0.336 & 0.042 & 0.253 & 0.419 & 0.125 & 4.988 & 641 \\
\hline Rural Lower Egypt & 0.698 & 0.018 & 0.662 & 0.735 & 0.026 & 2.894 & 1,618 & 0.425 & 0.019 & 0.388 & 0.462 & 0.044 & 2.343 & 1,808 \\
\hline Urban Upper Egypt & 0.659 & 0.067 & 0.526 & 0.791 & 0.102 & 9.169 & 289 & 0.455 & 0.045 & 0.366 & 0.544 & 0.099 & 3.505 & 350 \\
\hline Rural Upper Egypt & 0.696 & 0.030 & 0.637 & 0.755 & 0.043 & 6.532 & 1,110 & 0.394 & 0.025 & 0.345 & 0.443 & 0.063 & 4.157 & 1,509 \\
\hline Frontier Governorates & 0.520 & 0.049 & 0.424 & 0.616 & 0.094 & 0.975 & 455 & 0.257 & 0.031 & 0.195 & 0.319 & 0.122 & 0.500 & 496 \\
\hline \multicolumn{15}{|l|}{ Age group } \\
\hline 10-14 years & 0.767 & 0.018 & 0.732 & 0.802 & 0.023 & 1.932 & 1,091 & 0.566 & 0.020 & 0.526 & 0.605 & 0.036 & 1.845 & 1,105 \\
\hline $15-17$ years & 0.671 & 0.017 & 0.638 & 0.705 & 0.025 & 3.008 & 2,080 & 0.365 & 0.016 & 0.333 & 0.397 & 0.044 & 2.270 & 2,078 \\
\hline $18-24$ years & 0.616 & 0.022 & 0.573 & 0.658 & 0.035 & 2.570 & 1,108 & 0.355 & 0.018 & 0.320 & 0.389 & 0.049 & 1.667 & 1,456 \\
\hline $25-29$ years & 0.613 & 0.023 & 0.568 & 0.659 & 0.038 & 1.974 & 794 & 0.377 & 0.019 & 0.340 & 0.413 & 0.049 & 1.436 & 1,204 \\
\hline \multicolumn{15}{|l|}{ Education level } \\
\hline Illiterate & 0.620 & 0.043 & 0.535 & 0.705 & 0.070 & 2.521 & 288 & 0.350 & 0.027 & 0.297 & 0.403 & 0.077 & 2.127 & 831 \\
\hline Read and write & 0.763 & 0.087 & 0.591 & 0.934 & 0.115 & 1.657 & 38 & 0.340 & 0.064 & 0.214 & 0.466 & 0.188 & 1.233 & 83 \\
\hline Primary & 0.614 & 0.029 & 0.557 & 0.671 & 0.047 & 1.750 & 455 & 0.340 & 0.028 & 0.285 & 0.394 & 0.081 & 1.321 & 439 \\
\hline Preparatory & 0.672 & 0.025 & 0.623 & 0.721 & 0.037 & 1.989 & 646 & 0.458 & 0.022 & 0.414 & 0.503 & 0.049 & 1.645 & 872 \\
\hline General secondary & 0.778 & 0.024 & 0.730 & 0.827 & 0.031 & 1.514 & 417 & 0.533 & 0.027 & 0.479 & 0.587 & 0.051 & 1.465 & 516 \\
\hline Vocational secondary & 0.674 & 0.018 & 0.639 & 0.710 & 0.027 & 3.341 & 2,027 & 0.393 & 0.017 & 0.361 & 0.426 & 0.042 & 2.021 & 1,952 \\
\hline Post-secondary institute & 0.713 & 0.042 & 0.631 & 0.794 & 0.058 & 1.723 & 188 & 0.330 & 0.044 & 0.243 & 0.417 & 0.134 & 1.354 & 165 \\
\hline University and above & 0.638 & 0.020 & 0.598 & 0.678 & 0.032 & 2.086 & 1,014 & 0.410 & 0.023 & 0.364 & 0.456 & 0.057 & 2.245 & 982 \\
\hline \multicolumn{15}{|l|}{ Wealth quintile } \\
\hline Lowest & 0.672 & 0.026 & 0.620 & 0.724 & 0.039 & 3.112 & 829 & 0.381 & 0.025 & 0.333 & 0.430 & 0.065 & 2.620 & 1,021 \\
\hline Second & 0.645 & 0.026 & 0.593 & 0.696 & 0.041 & 3.359 & 954 & 0.422 & 0.022 & 0.378 & 0.465 & 0.052 & 2.134 & 1,145 \\
\hline Middle & 0.665 & 0.023 & 0.620 & 0.709 & 0.034 & 2.364 & 987 & 0.408 & 0.021 & 0.366 & 0.449 & 0.051 & 1.831 & 1,165 \\
\hline Fourth & 0.690 & 0.022 & 0.646 & 0.734 & 0.032 & 2.663 & 1,082 & 0.407 & 0.021 & 0.365 & 0.448 & 0.052 & 1.897 & 1,192 \\
\hline Highest & 0.669 & 0.019 & 0.632 & 0.707 & 0.028 & 2.142 & 1,221 & 0.416 & 0.020 & 0.377 & 0.455 & 0.048 & 1.984 & 1,320 \\
\hline
\end{tabular}

